\title{
A General Mechanism of Diffusion in Hamiltonian Systems: Qualitative Results
}

\author{
MARIAN GIDEA \\ Yeshiva University \\ RAFAEL DE LA LLAVE \\ Georgia Institute of Technology \\ AND \\ TERE M-SEARA \\ Universitat Politècnica de Catalunya, BGSMATH \\ Dedicated to the memory of Anatole Katok
}

\begin{abstract}
We present a general mechanism to establish the existence of diffusing orbits in a large class of nearly integrable Hamiltonian systems. Our approach is based on following the "outer dynamics" along homoclinic orbits to a normally hyperbolic invariant manifold. The information on the outer dynamics is encoded by a geometrically defined "scattering map." We show that for every finite sequence of successive iterations of the scattering map, there exists a true orbit that follows that sequence, provided that the inner dynamics is recurrent. We apply this result to prove the existence of diffusing orbits that cross large gaps in a priori unstable models of arbitrary degrees of freedom, when the unperturbed Hamiltonian is not necessarily convex and the induced inner dynamics is not necessarily a twist map, and the perturbation satisfies explicit conditions that are satisfied generically.

We also mention several other applications where this mechanism is easy to verify (analytically or numerically), such as the planar elliptic restricted threebody problem and the spatial circular restricted three-body problem.

Our method differs, in several crucial aspects, from earlier works. Unlike the well-known "two-dynamics" approach, the method we present here relies on the outer dynamics alone. There are virtually no assumptions on the inner dynamics, such as on existence of its invariant objects (e.g., primary and secondary tori, lower-dimensional hyperbolic tori, and their stable/unstable manifolds, AubryMather sets), which are not used at all. (c) 2019 Wiley Periodicals, Inc.
\end{abstract}

\section{Introduction}

\subsection{Brief Description of the Main Results}

Understanding the long-term behavior of nearly integrable Hamiltonian systems was viewed by Poincaré as the fundamental problem of dynamics. A major paradigm that emerged from this problem is the Arnold diffusion problem [1], asserting that "generic" integrable systems subjected to arbitrarily small, "generic" perturbations, have orbits that travel a distance that is independent of the size of the 
perturbation, henceforth referred to as diffusing orbits. In this sense, such orbits exhibit global instability, and their behavior is not captured by classical perturbation theory. In Section 1.2 we provide a brief overview of the work on this problem.

Finding explicit mechanisms of diffusion that can be verified in concrete models is of interest in applications, since it gives insights into how to produce large effects by applying small forces.

In this paper we develop a general but simple method to show the existence of diffusing orbits in nearly integrable Hamiltonian systems in any dimension. The main requirement for the system is to have a normally hyperbolic invariant manifold whose stable and unstable manifolds intersect transversally along a transverse homoclinic manifold. In this setting, one can geometrically define a map on the normally hyperbolic invariant manifold, referred to as the scattering map [32, 36], which accounts for the "outer" dynamics along homoclinic orbits. The scattering map assigns to the foot-point of an unstable fiber the foot-point of a stable fiber, provided the two fibers meet at a unique point in the homoclinic manifold. On the "inner dynamics," defined by the restriction to the normally hyperbolic invariant manifold, we only require that it satisfies Poincaré recurrence.

The main results of this paper can be summarized as follows:

(i) For every infinite pseudo-orbi $11^{1}$ generated by alternatively applying the scattering map and the inner dynamics for sufficiently many times, there exists a true orbit that shadows that pseudo-orbit. The statement is given in Lemma 3.1.

(ii) For every finite pseudo-orbit obtained by successively applying the scattering map, under the assumption that the inner dynamics satisfies Poincaré recurrence, there exists a true orbit that shadows it (its intermediate points follow the pseudo-orbit). The statement is given in Theorem 3.6 .

More precisely, every finite pseudo-orbit of the scattering map can be interspersed with arbitrarily long orbit segments of the inner dynamics, so that the previous shadowing lemma can be applied to obtain true orbits.

(iii) For a class of perturbed systems that satisfy some verifiable conditions, there exist pseudo-orbits of the scattering map that travel a distance of order $O(1)$ in the action variable; hence, by the previous shadowing result, there exist true orbits that follow them. The specific class of systems is described in Section 2.2, and the statement is given in Theorem 3.11 .

(iv) For a class of a priori unstable, nearly integrable Hamiltonian systems, as described in Section 4, the conditions that yield diffusing orbits occur generically.

More precisely, there exists a vector field on the normally hyperbolic invariant manifold whose integral curves are followed closely by pseudo-orbits of the scattering map. If this vector field is nontrivial at some point, we then show that the

\footnotetext{
${ }^{1}$ In this paper we use the term pseudo-orbit in the sense of an orbit of an iterated function system or poly-system; see $|74|$.
} 
corresponding integral curve travels a distance of order 1, and there is a pseudoorbit of the scattering map that goes along that curve.

The above results remain valid if one considers several transverse homoclinic manifolds rather than a single one, and hence several scattering maps. They also remain valid if one considers a sequence of manifolds (which may be of different topologies) chained via different heteroclinic connections, which can also be described via scattering maps.

For the purpose of establishing the existence of diffusing orbits, the assumption that the inner dynamics satisfies Poincaré recurrence on some bounded domain can be eliminated for Hamiltonian systems. If there is no such domain, then there exist diffusing orbits determined just by the inner dynamics.

As a concrete application of this method we obtain a qualitative result on the existence of diffusing orbits in a priori unstable Hamiltonian systems (see [24]) of any dimension, under verifiable conditions on the perturbation that are generically satisfied, and under some mild conditions on the unperturbed system.

In particular, the unperturbed Hamiltonian does not need to be convex, and it does not need to induce a twist map for the inner dynamics. The main requirement on the Hamiltonian system is that we can compute perturbatively the scattering map.

The salient features of the mechanism outlined above are the following:

(1) We do not require any information on the inner dynamics. In particular, we can obtain diffusing orbits whose action variable crosses resonant surfaces of any multiplicity.

This is a significant departure from previous approaches that rely on a detailed analysis of the invariant objects for the inner dynamics: primary KAM tori, secondary tori, lower-dimensional hyperbolic tori and their stable and unstable manifolds, Aubry-Mather sets, etc. In fact, we do not need the inner dynamics to satisfy a twist condition, which is a key assumption in previous geometric and variational approaches. In particular, the present mechanism does not present the large gap problem.

(2) The normally hyperbolic invariant manifold as well as its stable and unstable manifolds can be of arbitrary dimensions.

(3) We can take advantage of the existence of several scattering maps.

(4) Our method can be applied to concrete systems-e.g., the planar elliptic restricted three-body problem, and the spatial circular restricted three-body problem - and, further, can be implemented in computer-assisted proofs. See the related papers [19,40,44].

(5) Although the main application in this paper is on diffusion in a priori unstable systems, we expect that this method can be useful when applied to a priori stable systems, as well as to infinite-dimensional systems, once the existence of suitable normally hyperbolic invariant manifolds (called normally hyperbolic cylinders in [6, 68, 69, 75]) and their homoclinic channels is established. See Remark 3.16 . 


\subsection{Related Works}

We compare our method here with some previous approaches to the diffusion problem for different types of Hamiltonian systems.

It is customary to distinguish between geometric methods and variational methods. The method in this paper is geometric, so we first compare it with some related approaches.

For nearly integrable Hamiltonian systems of two-and-a-half degrees of freedom, the existence of diffusion has been established via geometric methods in [32,34] by using the existence of KAM tori, primary and secondary, along the normally hyperbolic invariant manifold. The perturbation in [34] is assumed to be a trigonometric polynomial in the angle variable, but [42] eliminates this assumption. The integrable Hamiltonian is not assumed to be convex, which seems to be the standard assumption in many variational approaches. A similar type of results has been obtained in [53, 54] with the use of the method of correctly aligned windows. This allows us to simplify the proofs and to obtain explicit estimates on the diffusion speed.

The case of higher-dimensional Hamiltonian systems poses a difficulty that is not present in the case of two-and-a-half degrees of freedom: there are points in the normally hyperbolic invariant manifold where the resonances have higher multiplicity. The technique involved in [32,34] uses heavily that in the neighborhood of resonances of multiplicity 1 one can introduce a normal form that is integrable and can be analyzed with great accuracy to obtain secondary tori. Unfortunately, it is well-known that multiple resonances, that is, resonances of multiplicity greater than or equal to 2, lead to normal forms that are not integrable and require other techniques to be analyzed (see [63,64]).

In [37] the authors adapted the methods used in two-and-a-half degrees of freedom to show instability in higher dimensions. Their approach relies on the basic fact that multiple resonances happen in subsets of codimension greater than 1 in the space of actions and therefore the diffusing trajectories can contour them.

A key idea of our paper is that our mechanism relies mostly on following the outer dynamics, and it does not need any detailed information on the inner dynamics, such as invariant tori and resonance webs, as we only need that the inner dynamics be recurrent. This is automatically satisfied in the Hamiltonian case. Some other works in Hamiltonian dynamics in which recurrence property plays an important role include [56, 57, 79].

A work closely related to ours is [52], in which the authors obtain a shadowing lemma for finite pseudo-orbits and use it to show the existence of diffusing orbits in a priori chaotic systems. Their shadowing lemma also does not use information on the inner dynamics and relies on Poincaré recurrence. We note that their shadowing lemma can alternatively be used to show the existence of true orbits that follow the diffusing pseudo-orbits obtained in the proof of Theorem 3.11. There is a notable difference: our shadowing lemma, Lemma 3.1, works for infinite pseudo-orbits, 
and can be used to show the existence of symbolic dynamics as well as of orbits that escape to infinity.

Now we mention some other types of approaches to the diffusion problem.

Geometric methods based on normally hyperbolic invariant manifolds that use the separatrix map rather than the scattering map appear in [15, 81, 82, 89,-92]. Other geometric methods have been applied in [16, 28, 33, 38, 51, 67, 75, 95]. These geometric methods also work for systems with magnetic fields [73] and for dissipative systems [61].

Several authors have used variational methods (either local variational methods or global variational methods) alone or in combination with geometric methods, to obtain results on diffusion. This is the case, for example, in [5-12, 20,-23, 68,-70, [77,78]. We mention the paper [88], which suggests several other mechanisms that should be at play. It seems to be a very challenging problem to make rigorous the heuristic discussions on statistical and quantitative properties of different instability mechanisms in the heuristic literature [25, 71, 88].

We also acknowledge that many of the methods and ideas that appear in the works on the Arnold diffusion problem are owed to John Mather, whose influence to the field cannot be overstated [76-78].

\subsection{Structure of the Paper}

In Section 2 we review some background on normally hyperbolic invariant manifolds, the scattering map, and recurrent dynamics. In Section 3 we provide two general results on the existence of diffusing orbits, Theorem 3.6 and Theorem 3.11 . as well as some corollaries. We also provide a general shadowing lemma, Lemma 3.1. that is used in proving these results. An application to establish the existence of diffusing orbits in a class of nearly integrable a priori unstable Hamiltonian systems that are multi-dimensional both in the center and in the hyperbolic directions is given in Section 4. A novelty is that the unperturbed system corresponds to a Hamiltonian that is not necessarily convex, and that the inner dynamics does not need to satisfy a twist condition. Section 5 contains the proofs of the results stated in Section 3. In particular, for the shadowing result given by Lemma 3.1 we give two proofs, one based on correctly aligned windows and the other on the obstruction argument; while the first one is more constructive, the second one is simpler. The appendices contain definitions and tools that are utilized in the paper; they are included for the convenience of the reader. We also present in Appendix $\mathrm{D}$ an example communicated to us by D. Turaev showing that a uniform version of Lemma 3.1 is not possible.

\section{Background}

In this section, we cover some standard material that will be used in the statement of the results. All the material will be well-known to experts. 


\subsection{Normally Hyperbolic Invariant Manifolds and Scattering Maps}

Consider a discrete-time dynamical system given by the action of a $C^{r}$-smooth map $f$ on a $C^{r}$-smooth manifold $M$ of dimension $m$, where $r \geq 1$.

Assume that $\Lambda$ is a normally hyperbolic invariant manifold (NHIM) in $M$, of dimension $n_{c}$ : this means that the tangent bundle of $M$ restricted to $\Lambda$ splits as a Whitney sum of subbundles $T M_{\mid \Lambda}=T \Lambda \oplus E^{u} \oplus E^{s}$ that are invariant under $D f$, and that $(D f)_{\mid E^{u}}$ expands more than $(D f)_{\mid T \Lambda}$, while $(D f)_{\mid E^{s}}$ contracts more than $(D f)_{\mid T \Lambda}$. We also assume that $\Lambda$ is compact or that $f$ is uniformly $C^{r}$ in a neighborhood of $\Lambda$. The rather standard definition of the NHIM is given in Appendix A.

In the sequel we assume that the stable and unstable bundles associated to the normally hyperbolic structure have dimensions $n_{u}, n_{s}>0$, respectively, where $n_{c}+n_{u}+n_{s}=m$. (In many applications concerning diffusion in nearly integrable Hamiltonian systems, we have $n_{u}=n_{s}=n$ and $n_{c}=$ even number, hence $m=$ even number.)

Remark 2.1. In the general theory of normally hyperbolic manifolds one does not have the above restriction on dimensions, but for symplectic systems, this is natural. We also note that in the symplectic case, it is natural to assume that the stable and unstable rates [49] and that the forward rates in the tangent direction are the same. In such a case, one has automatically that the invariant manifold is symplectic. See [36].

The normal hyperbolicity of $\Lambda$ implies that there exist stable and unstable invariant manifolds, $W^{s}(\Lambda)$ and $W^{u}(\Lambda)$, of $\Lambda$. The exponential contraction and expansion rates of $D f$ along the stable and unstable bundles, and on $T \Lambda$, determine an integer $\ell$ with $0<\ell \leq r$ such that $\Lambda, W^{s}(\Lambda)$, and $W^{u}(\Lambda)$ are $C^{\ell}$-smooth manifolds. The stable and unstable manifolds $W^{s}(\Lambda)$ and $W^{u}(\Lambda)$ are foliated by stable and unstable fibers $W^{s}(x)$ and $W^{u}(x)$, respectively, with $x \in \Lambda$, which are $C^{r}$-smooth one-dimensional manifolds. The corresponding foliations are, however, only $C^{\ell-1}$-smooth. See Appendix A

From now on we assume that $r$ and the normally hyperbolic structure are so that $\ell \geq 2$.

Let $\Gamma \subseteq W^{s}(\Lambda) \cap W^{u}(\Lambda)$ be a $C^{\ell-1}$-smooth homoclinic manifold. Consider the wave maps

$$
\begin{aligned}
& \Omega^{-}: \Gamma \subset W^{u}(\Lambda) \rightarrow \Omega^{-}(\Gamma) \subseteq \Lambda, \Omega^{-}(x)=x^{-}, \\
& \Omega^{+}: \Gamma \subset W^{s}(\Lambda) \rightarrow \Omega^{+}(\Gamma) \subseteq \Lambda, \Omega^{+}(x)=x^{+},
\end{aligned}
$$

where $x^{-}$is the unique point in $\Lambda$ such that $x \in W^{u}\left(x^{-}\right)$and $x^{+}$is the unique point in $\Lambda$ such that $x \in W^{s}\left(x^{+}\right)$.

Under certain restrictions on $\Gamma$, which amount to the strong transversality condition A.7) and choosing $\Gamma$ sufficiently small (see Appendix A , the wave maps $\Omega^{ \pm}$ are $C^{\ell-1}$-diffeomorphisms from $\Gamma$ to their images. Such a homoclinic manifold $\Gamma$ is referred to as a homoclinic channel. 
Assuming that $\Gamma$ is a homoclinic channel, one can define a $C^{\ell-1}$ diffeomorphism

$$
\sigma: \Omega^{-}(\Gamma) \rightarrow \Omega^{+}(\Gamma) \text { given by } \sigma=\Omega^{+} \circ\left(\Omega^{-}\right)^{-1} \text {, }
$$

where $\Omega^{-}(\Gamma)$ and $\Omega^{+}(\Gamma)$ are open sets in $\Lambda$. That is, $\sigma\left(x^{-}\right)=x^{+}$for every $x^{-} \in \Omega^{-}(\Gamma)$ defined as above. The mapping $\sigma$ is referred to as the scattering map associated to the homoclinic channel $\Gamma$. For details on this setup and general properties of the scattering map, see Appendix A.

We shall note that there is no actual orbit of the system that goes from $x^{-}$to $\sigma\left(x^{-}\right)=x^{+}$. Rather, the geometric object that corresponds to $\sigma\left(x^{-}\right)=x^{+}$is the heteroclinic orbit $\left\{f^{n}(x)\right\}_{n \in \mathbb{Z}}$ of $x$, which approaches asymptotically $f^{n}\left(x^{-}\right)$ backwards in time as $n \rightarrow-\infty$ and $f^{n}\left(x^{+}\right)$forward in time as $n \rightarrow+\infty$. We remark that, if we denote by $\sigma^{\Gamma}$ the scattering map associated to the homoclinic channel $\Gamma$, then for each $k \in \mathbb{Z}, f^{k}(\Gamma)$ is also a homoclinic channel, and the corresponding scattering map $\sigma^{f^{k}(\Gamma)}$ is related to $\sigma^{\Gamma}$ by the invariance relation

$$
\sigma^{f^{k}(\Gamma)}=f^{k} \circ \sigma^{\Gamma} \circ f^{-k}
$$

While $\sigma^{\Gamma}$ and $\sigma^{f^{k}(\Gamma)}$ are technically different scattering maps, they are geometrically the same, as they are defined via the same homoclinic channel (up to iterations by the map $f$ ). Of course, homoclinic channels that are not obtained from one another via iteration yield, in general, two scattering maps that are geometrically different.

In many examples, the scattering map can be computed explicitly via perturbation theory [31, 34, 35] or numerically [18, 19, 39, 40].

\subsection{Normally Hyperbolic Invariant Manifolds and Scattering Maps in a Symplectic Perturbative Setting}

Assume now that $(M, \omega)$ is a symplectic manifold and $f_{\varepsilon}: M \rightarrow M$ is a $C^{r}$ family of symplectic maps, where $\varepsilon \in\left(\varepsilon_{0}, \varepsilon_{0}\right)$ for some $\varepsilon_{0}>0$.

As an example, $f_{\varepsilon}$ can be the time-1 map associated to the Hamiltonian flow $\phi_{t, \varepsilon}$ corresponding to a Hamiltonian $H_{\varepsilon}: M \rightarrow \mathbb{R}$ of the form

$$
H_{\varepsilon}=H_{0}+\varepsilon H_{1} \text {. }
$$

In this case, the maps $f_{\varepsilon}$ with $\varepsilon \neq 0$ can be viewed as $\varepsilon$-perturbations of the map $f_{0}$, the time-1 map for the unperturbed Hamiltonian flow of $H_{0}$.

Going back to the general setting, assume that there exists a normally hyperbolic invariant manifold $\Lambda_{\varepsilon} \subseteq M$ for $f_{\varepsilon}$, of even dimension $n_{c}$, for all $\varepsilon \in\left(-\varepsilon_{0}, \varepsilon_{0}\right)$, and that $\operatorname{dim} W^{u}\left(\Lambda_{\varepsilon}\right)=n_{c}+n_{u}=\operatorname{dim} W^{s}\left(\Lambda_{\varepsilon}\right)=n_{c}+n_{s}$. Assume that $\Lambda_{\varepsilon}$ is symplectic and denote by $J$ the linear operator associated to $\omega_{\mid \Lambda_{\varepsilon}}$ by the metric. Then the map $f_{\varepsilon}$ is also symplectic on $\Lambda_{\varepsilon}$.

Assume for each $\varepsilon \in\left(-\varepsilon_{0}, \varepsilon_{0}\right)$ there exists a homoclinic channel $\Gamma_{\varepsilon}$ for $f_{\varepsilon}$ that depends $C^{\ell-1}$-smoothly on $\varepsilon$. Then the scattering map $\sigma_{\varepsilon}: \Omega^{-}\left(\Gamma_{\varepsilon}\right) \rightarrow \Omega^{-}\left(\Gamma_{\varepsilon}\right)$ is also symplectic and $C^{\ell-1}$ (see [36, 38]). 
Now we assume that $\Lambda_{\varepsilon}$ can be parametrized via a symplectic $C^{\ell}$-diffeomorphism $k_{\varepsilon}: \Lambda_{0} \rightarrow \Lambda_{\varepsilon}$ for $\varepsilon \in\left(-\varepsilon_{0}, \varepsilon_{0}\right)$, where $\Lambda_{0}$ is the normally hyperbolic manifold for the unperturbed map $f_{0}$, and $k_{0}=\operatorname{Id}_{\Lambda_{0}}$. This happens, for example, when the $\Lambda_{\varepsilon}$ 's are obtained by the persistence of normal hyperbolicity under sufficiently small perturbations (see [34]).

Via the parametrizations $k_{\varepsilon}$, each map $f_{\varepsilon}$ induces a map $\tilde{f}_{\varepsilon}$ on $\Lambda_{0}$ by

$$
\tilde{f_{\varepsilon}}=k_{\varepsilon}^{-1} \circ\left(f_{\varepsilon}\right)_{\mid \Lambda_{\varepsilon}} \circ k_{\varepsilon}
$$

The scattering map $\sigma_{\varepsilon}: \Omega^{-}\left(\Gamma_{\varepsilon}\right) \subset \Lambda_{\varepsilon} \rightarrow \Omega^{+}\left(\Gamma_{\varepsilon}\right) \subset \Lambda_{\varepsilon}$ can also be expressed in terms of the reference manifold $\Lambda_{0}$ by

$$
\tilde{\sigma}_{\varepsilon}: k_{\varepsilon}^{-1}\left(\Omega^{-}\left(\Gamma_{\varepsilon}\right)\right) \subset \Lambda_{0} \rightarrow k_{\varepsilon}^{-1}\left(\Omega^{+}\left(\Gamma_{\varepsilon}\right)\right) \subset \Lambda_{0}
$$

given by

$$
\widetilde{\sigma}_{\varepsilon}=k_{\varepsilon}^{-1} \circ \sigma_{\varepsilon} \circ k_{\varepsilon} .
$$

We will refer to the map $\widetilde{\sigma}_{\varepsilon}$ also as the scattering map.

In this setting, one also has an unperturbed scattering map $\sigma_{0}$ on the unperturbed manifold $\Lambda_{0}$, associated to the homoclinic channel $\Gamma_{0}$ contained in the intersection between the stable and unstable manifolds of $\Lambda_{0}$. Of course, in the unperturbed case one has $\tilde{\sigma}_{0}=k_{0}^{-1} \circ \sigma_{0} \circ k_{0}=\sigma_{0}$. Expressing both the perturbed and the unperturbed scattering map as maps on the same (unperturbed) manifold is quite advantageous, as one can compare them relative to the same coordinate system.

For a Hamiltonian system $H_{\varepsilon}$ as in [2.2], [36] provides a perturbative formula for the scattering map:

$$
\tilde{\sigma}_{\varepsilon}=\tilde{\sigma}_{0}+\varepsilon J \nabla S \circ \tilde{\sigma}_{0}+O\left(\varepsilon^{2}\right)
$$

where $S$ is a real-valued $C^{\ell}$-function on $\Lambda_{0}$ that can be computed explicitly in terms of convergent integrals of the perturbation evaluated along homoclinic trajectories of the unperturbed system (see [36, 55]):

$$
\begin{aligned}
& S(x)=\lim _{T \rightarrow+\infty} \int_{-T}^{0}\left[\frac{d H_{\varepsilon}}{d \varepsilon}{ }_{\mid \varepsilon=0} \circ \phi_{t} \circ\left(\Omega_{-}^{\Gamma_{0}}\right)^{-1} \circ \sigma_{0}^{-1}(x)\right. \\
& \left.-\left.\frac{d H_{\varepsilon}}{d \varepsilon}\right|_{\varepsilon=0} \circ \phi_{t} \circ \sigma_{0}^{-1}(x)\right] d t \\
& +\lim _{T \rightarrow+\infty} \int_{0}^{T}\left[\left.\frac{d H_{\varepsilon}}{d \varepsilon}\right|_{\varepsilon=0} \circ \phi_{t} \circ\left(\Omega_{+}^{\Gamma_{0}}\right)^{-1}(x)\right. \\
& \left.-\frac{d H_{\varepsilon}}{d \varepsilon \mid \varepsilon=0}{ }_{|c|} \circ \phi_{t}(x)\right] d t \text {. }
\end{aligned}
$$

Here $\phi_{t}=\phi_{t, 0}$ is the flow corresponding to the unperturbed Hamiltonian $H_{0}$.

Note that, by definition, there exists $z \in \Gamma_{0}$ such that $\phi_{t}(z)$ is a heteroclinic orbit, or, equivalently, $z \in W^{u}\left(\left(\sigma_{0}\right)^{-1}(x)\right) \cap W^{s}(x)$. Therefore, the formula (2.4) 
can also be written as

$$
\begin{aligned}
S(x)= & \lim _{T \rightarrow+\infty} \int_{-T}^{0}\left[\left.\frac{d H_{\varepsilon}}{d \varepsilon}\right|_{\varepsilon=0} \circ \phi_{t}(z)-\left.\frac{d H_{\varepsilon}}{d \varepsilon}\right|_{\varepsilon=0} \circ \phi_{t}\left(\sigma_{0}^{-1}(x)\right)\right] d t \\
& +\lim _{T \rightarrow+\infty} \int_{0}^{T}\left[\left.\frac{d H_{\varepsilon}}{d \varepsilon}\right|_{\varepsilon=0} \circ \phi_{t}(z)-\left.\frac{d H_{\varepsilon}}{d \varepsilon}\right|_{\mid \varepsilon=0} \circ \phi_{t}(x)\right] d t .
\end{aligned}
$$

The normal hyperbolicity of $\Lambda_{0}$ ensures that $\phi_{t}(z)-\phi_{t}\left(\sigma_{0}^{-1}(x)\right)$ and $\phi_{t}(z)-\phi_{t}(x)$ converge to 0 exponentially fast as $t \rightarrow \mp \infty$, respectively. This makes the integral in (2.5) absolutely convergent with its derivatives.

In some cases it is possible that, when $\varepsilon=0$, the stable and unstable manifolds of $\Lambda_{0}$ coincide, i.e., $W^{u}\left(\Lambda_{0}\right)=W^{s}\left(\Lambda_{0}\right)$; see, e.g., Section 4. In these cases, one usually uses first-order perturbation theory to establish the splitting of the manifolds. Using an adapted Melnikov method, in [34, 55] it is shown that, under appropriate conditions, for $0<|\varepsilon| \ll 1$, one can find a transverse intersection of $W^{u}\left(\Lambda_{\varepsilon}\right)$ with $W^{s}\left(\Lambda_{\varepsilon}\right)$ along a manifold $\Gamma_{\varepsilon}$, which extends smoothly to a homoclinic manifold $\Gamma_{0}$ as $\varepsilon \rightarrow 0$. While the limiting manifold $\Gamma_{0}$ is not a transverse intersection, the scattering map $\sigma_{\varepsilon}$ depends smoothly on $\varepsilon$, and thus extends smoothly to a well-defined map $\sigma_{0}$ associated to $\Gamma_{0}$.

The special case when $\sigma_{0}=\mathrm{Id}$, which occurs in many examples, will be considered in Section 4, where a more explicit formula for the function $S(x)$ is given in terms of the so-called Melnikov potential.

\subsection{Recurrence}

We briefly recall here the definition of recurrent points and the Poincaré recurrence theorem, which will be needed later.

Definition 2.2. A point $x \in \Lambda$ is said to be recurrent for a map $f$ on $\Lambda$ if for every open neighborhood $U \subseteq \Lambda$ of $x, f^{k}(x) \in U$ for some $k>0$ large enough.

THEOREM 2.3 (Poincaré recurrence theorem). Suppose that $\mu$ is a measure on $\Lambda$ that is preserved by $f$, and $D \subset \Lambda$ is $f$-invariant with $\mu(D)<\infty$. Then $\mu$-almost every point of $D$ is recurrent.

Instead of recurrent points, in the arguments below we can use nonwandering points.

Proposition 2.4. Suppose that $\mu$ is a measure on $\Lambda$ that is preserved by $f$, and $D \subset \Lambda$ is $f$-invariant with $\mu(D)<\infty$. Then every point $x \in D$ is nonwandering; that is, for every open neighborhood $U$ of $x$ in $D$, there exists $n \geq 1$ such that $f^{n}(U) \cap U \neq \varnothing$; moreover, $n$ can be chosen arbitrarily large.

\section{Main Results}

The aim of this section is to provide a master theorem, Theorem 3.6, that will be used to yield, in Theorem 3.11 and Corollary 3.12, the existence of diffusing orbits in a general framework. 


\subsection{Shadowing of Pseudo-orbits Obtained by Interspersing the Inner Dynamics with Scattering Maps}

In this section we provide a rather general-shadowing-lemma type of result that is needed for the proof of Theorem 3.6.

Let $\Lambda$ be an NHIM as in Section 2.1. There are two maps defined acting on $\Lambda$ : the scattering map $\sigma$, the outer dynamics, which is typically defined on some subdomain of $\Lambda$, called $\Omega^{-}(\Gamma)$ in Section 2.2, and the restriction of $f$ to $\Lambda$-the inner dynamics. In principle, one can act on $\Lambda$ by applying either map in any succession; however, this does not yield true orbits of the system but only pseudo-orbits.

The shadowing lemma below says that for every pseudo-orbit obtained by alternately applying a single scattering map and some sufficiently high power of the inner map, there exists a true orbit of the system that shadows that pseudo-orbit. The pseudo-orbits that we consider are of the form $y_{i+1}=f^{m_{i}} \circ \sigma \circ f^{n_{i}}\left(y_{i}\right)$. The resulting shadowing orbits are of the form $z_{i+1}=f^{m_{i}+n_{i}}\left(z_{i}\right)$, where $z_{i}$ is $\delta$-close to $y_{i}$ for all $i$. We point out that we do not claim that all points of the orbit $\left\{f^{n}\left(z_{0}\right)\right\}_{n \geq 0}$ are close to those of the pseudo-orbit, but only some points corresponding to some intermediate times, and this is the sense in which we understand shadowing orbits here.

The orders of the iterates $n_{i}$ and $m_{i}$ are required to satisfy certain conditions. Each power $n_{i}$ is required to be larger than some threshold value $n^{*}$, which depends on $\delta$, and each power $m_{i}$ is required to be larger than some threshold value $m_{i}^{*}$, which depends on the history of the pseudo-orbit up to that point, that is, on all previous powers $n_{0}, \ldots, n_{i-1}, n_{i}, m_{0}, \ldots, m_{i-1}$ that were utilized in the previous segments of the pseudo-orbit from $y_{0}$ to $y_{i}$. Intuitively, $m_{i}, n_{i}$ quantify the lengths of time for which we follow a homoclinic trajectory associated to the scattering map forward and backward in time, respectively, from $\Gamma$ to a neighborhood of $\Lambda$.

LEMMA 3.1 (Shadowing lemma for pseudo-orbits of the scattering map and the inner dynamics). Assume that $f: M \rightarrow M$ is a $C^{r}$-map, $r \geq r_{0}, \Lambda \subseteq M$ is a normally hyperbolic invariant manifold, $\Gamma \subseteq M$ is a homoclinic channel, and $\sigma^{\Gamma}: \Omega^{-}(\Gamma) \rightarrow \Omega^{+}(\Gamma)$ is the scattering map associated to $\Gamma$. Assume that $\Lambda$ and $\Gamma$ are compact.

Then, for every $\delta>0$ there exists $n^{*} \in \mathbb{N}$ depending on $\delta$ and a family of functions $m_{i}^{*}: \mathbb{N}^{2 i+1} \rightarrow \mathbb{N}, i \geq 0$, depending on $\delta$, such that, for every pseudoorbit $\left\{y_{i}\right\}_{i \geq 0}$ in $\Lambda$ of the form

$$
y_{i+1}=f^{m_{i}} \circ \sigma^{\Gamma} \circ f^{n_{i}}\left(y_{i}\right)
$$

for all $i \geq 0$, with $n_{i} \geq n^{*}$ and $m_{i} \geq m_{i}^{*}\left(n_{0}, \ldots, n_{i-1}, n_{i}, m_{0}, \ldots, m_{i-1}\right)$, there exists an orbit $\left\{z_{i}\right\}_{i \geq 0}$ of $f$ in $M$ such that, for all $i \geq 0$,

$$
z_{i+1}=f^{m_{i}+n_{i}}\left(z_{i}\right) \text { and } d\left(z_{i}, y_{i}\right)<\delta .
$$

The proof of Lemma 3.1 is given in Section 5.1.

Notice that the functions $n^{*}$ and $m_{i}^{*}$ are defined only after we choose $\delta$, so they depend on $\delta$. We emphasize that the sequence $y_{i}$ in $(3.1)$ is contained in $\Lambda$ so that 
the map $f$ that appears in the definition of $y_{i}$ can be taken to be $\left.f\right|_{\Lambda}$. The reason why we refer to the sequence $\left\{y_{i}\right\}$ in (3.1) as a "pseudo-orbit" is that $y_{i+1}$ and $y_{i}$ are close to the endpoints of a segment orbit of the full map.

Indeed, if we consider the point $p_{i}=\left(\Omega_{-}^{\Gamma}\right)^{-1} f^{n_{i}}\left(y_{i}\right)$, we see that $f^{-n_{i}}\left(p_{i}\right)$ and $f^{-n_{i}}\left(f^{n_{i}}\left(y_{i}\right)\right)=y_{i}$ would be close since they are in the same unstable fiber and $n_{i}$ is large. We also see that $\sigma^{\Gamma} f^{n_{i}}\left(y_{i}\right)=\Omega_{+}^{\Gamma}\left(p_{i}\right)$. Therefore, $f^{m_{i}}\left(p_{i}\right)$ and $f^{m_{i}} \circ \sigma^{\Gamma} \circ f^{n_{i}}\left(y_{i}\right)$ will be close since they are in the same stable fiber.

Therefore, the sequence $\left\{y_{i}\right\}$ is approximated by a concatenation of segments of orbits $\mathcal{O}_{i}=\left\{f^{j}\left(p_{i}\right)\right\}_{j=-n_{i}}^{m_{i}}$. The mismatches at the ends of these segments of orbits are clearly small.

It would be natural to try to use a hyperbolic shadowing theorem to follow this pseudo-orbit. Unfortunately, with the present hypothesis, we do not have any information on the expanding or contracting properties of the map along the directions tangent to $\Lambda$, and standard hyperbolic shadowing theorems do not seem to apply. We have to give a different proof and introduce the condition that the $m_{i}$ 's grow.

Lemma 3.1 can be immediately extended to the case of countably many scattering maps. Suppose that there exists an infinite collection of homoclinic channels $\Gamma_{j} \subseteq M$, for $j \in \mathbb{N}$, and let

$$
\sigma_{j}: \Omega^{-}\left(\Gamma_{j}\right) \rightarrow \Omega^{+}\left(\Gamma_{j}\right)
$$

be the scattering map associated to $\Gamma_{j}$ for $j \in \mathbb{N}$.

LEMMA 3.2. Assume that $f: M \rightarrow M, \Lambda \subseteq M, \Gamma_{j} \subseteq M$, and $\sigma_{j}$ are as above for $j \in \mathbb{N}$. Assume that $\Lambda$ and $\Gamma_{j}$ are compact.

Then, for every $\delta>0$ there exist two families of functions, $n_{i}^{*}: \mathbb{N}^{i} \rightarrow \mathbb{N}$ and $m_{i}^{*}: \mathbb{N}^{2 i+1} \times \mathbb{N}^{i+1} \rightarrow \mathbb{N}$, both depending on $\delta$, for $i \geq 0$, such that, for every pseudo-orbit $\left\{y_{i}\right\}_{i \geq 0}$ in $\Lambda$ of the form

$$
y_{i+1}=f^{m_{i}} \circ \sigma_{\alpha_{i}} \circ f^{n_{i}}\left(y_{i}\right)
$$

where $n_{i} \geq n^{*}\left(\alpha_{0}, \ldots, \alpha_{i-1}\right), m_{i} \geq m^{*}\left(n_{0}, \ldots, n_{i}, m_{0}, \ldots, m_{i-1}, \alpha_{0}, \ldots, \alpha_{i}\right)$ for all $i \geq 0$, there exists an orbit $\left\{z_{i}\right\}_{i \geq 0}$ of $f$ in $M$ such that, for all $i \geq 0$,

$$
z_{i+1}=f^{m_{i}+n_{i}}\left(z_{i}\right) \text { and } d\left(z_{i}, y_{i}\right)<\delta .
$$

Remark 3.3. Even if it is not explicitly written in Lemma 3.1, $n^{*}$ and $m_{i}^{*}$ also depend on the hyperbolic structure, and in particular on the angle of intersection between $W^{u}(\Lambda)$ with $W^{s}(\Lambda)$ along $\Gamma$.

Remark 3.4. Note that Lemma 3.1 does not use any symplectic structure. It is valid for general maps. Hence, the results obtained from it remain valid for dissipative perturbations of Hamiltonian systems. Of course, when the perturbations are Hamiltonian we can obtain stronger results.

Remark 3.5. We note that results related to Lemma 3.1 appear in [39, 52, 60]. In comparison to our lemma, [39,60] make some geometric assumptions on the inner dynamics, and [52] considers only finite pseudo-orbits. 


\subsection{Shadowing of Pseudo-orbits of the Scattering Map}

The main result of this section is the following:

THEOREM 3.6 (Shadowing Lemma for Orbits of the Scattering Map). Assume that $f: M \rightarrow M$ is a sufficiently smooth map, $\Lambda \subseteq M$ is a normally hyperbolic invariant manifold with stable and unstable manifolds that intersect transversally along an homoclinic channel $\Gamma \subseteq M$, and $\sigma$ is the scattering map associated to $\Gamma$.

Assume that $f$ preserves a measure absolutely continuous with respect to the Lebesgue measure on $\Lambda$, and that $\sigma$ sends positive measure sets to positive measure sets.

Let $\left\{x_{i}\right\}_{i=0, \ldots, n}$ be a finite pseudo-orbit of the scattering map in $\Lambda$, i.e., $x_{i+1}=$ $\sigma\left(x_{i}\right), i=0, \ldots, n-1, n \geq 1$, which is contained in some open set $\mathcal{U} \subseteq \Lambda$ with almost every point of $\mathcal{U}$ recurrent for $f_{\mid \Lambda}$. (The points $\left\{x_{i}\right\}_{i=0, \ldots, n}$ do not have to be themselves recurrent.)

Then, for every $\delta>0$ there exists an orbit $\left\{z_{i}\right\}_{i=0, \ldots, n}$ of $f$ in $M$, with $z_{i+1}=$ $f^{k_{i}}\left(z_{i}\right)$ for some $k_{i}>0$, such that $d\left(z_{i}, x_{i}\right)<\delta$ for all $i=0, \ldots, n$.

The proof of this result, given in Section 5.2, uses the given pseudo-orbit of the scattering map and the recurrence property of the inner dynamics to produce another pseudo-orbit that intertwines the scattering map and the inner dynamics. Then, the shadowing lemma, Lemma 3.1, yields a true orbit of the system.

To apply Theorem 3.6, one needs to find orbits of the scattering map that follow desired itineraries. For example, one may wish to find a pseudo-orbit of the scattering map that travels a "long distance" in $\Lambda$. If such a pseudo-orbit is found, Theorem 3.6 yields a true orbit that also travels the same large distance.

We emphasize that Theorem 3.6 is very general, as the requirements on the scattering map and on the inner dynamics are automatically satisfied in many situations. If $M$ is endowed with a symplectic form $\omega, \omega_{\mid \Lambda}$ is symplectic, and $f$ is also symplectic, then $f_{\mid \Lambda}$ is symplectic and the scattering map $\sigma$ is also symplectic (see [36]). Thus, $f$ and $\sigma$ are volume preserving, and Theorem 3.6 applies.

We have the following remarkable dichotomy. Either:

I. The inner map $f_{\mid \Lambda}$ has an invariant open set $\mathcal{U}$ containing the domain of the scattering map and on which there is Poincaré recurrence. Under generic conditions, the scattering map has a pseudo-orbit that travels a long distance within $\mathcal{U}$. Applying Theorem 3.6 yields the existence of a true orbit that travels a long distance as well. Therefore we obtain diffusion by intertwining the inner and outer dynamics.

II. There is no open set of finite measure in $\Lambda$ that is invariant under $f_{\mid \Lambda}$. Hence there are orbits of $f$ that leave every open set in $\Lambda$, thus traveling long distances. Therefore we obtain diffusion by the inner map $f_{\mid \Lambda}$ alone.

In both alternatives we obtain diffusing orbits.

A precise formulation of this dichotomy is given in Corollary 3.12 .

Note that in Theorem 3.6 we do not require that $f$ satisfy a twist condition, which seems to be essential in many other works. In general, nontwist maps of 
the annulus have regions where standard methods such as KAM theory and AubryMather theory do not apply (see [30,31]).

Indeed, in Theorem 3.6, we do not need to make any qualitative assumption about the map $f$. In particular, we do not care whether the map has KAM tori that are close enough. That is, the mechanism presented here does not present the large gap problem.

Theorem 3.6 extends naturally to the case of finitely many scattering maps rather than a single one. Suppose that there exists a finite collection of homoclinic channels $\Gamma_{j} \subseteq M$, for $j \in\{1, \ldots, L\}$, for some positive integer $L$. Let $\sigma_{j}: \Omega^{-}\left(\Gamma_{j}\right) \rightarrow \Omega^{+}\left(\Gamma_{j}\right)$ be the scattering map associated to $\Gamma_{j}$ for $j=1, \ldots, L$.

Using many scattering maps in arbitrary order rather than just one is very advantageous in proving diffusion. Iterating a single map has obstructions for large-scale motions (e.g., KAM tori). Having several maps, it is very hard to find objects that are invariant for all of them. See [13, 14, 32, 54, 56, 59, 74].

Theorem 3.7. Assume that $f: M \rightarrow M, \Lambda \subseteq M, \Gamma_{j} \subseteq M$, and $\sigma_{j}, j=$ $1, \ldots, L$, are as above. Assume that $f$ preserves a measure absolutely continuous with respect to the Lebesgue measure on $\Lambda$ and that each $\sigma_{j}$ sends positive measure sets to positive measure sets. Let $\left\{x_{i}\right\}_{i=0, \ldots, n}$ be a finite sequence of points of the form $x_{i+1}=\sigma_{\alpha_{i}}\left(x_{i}\right)$ in $\Lambda$, where $\alpha_{i} \in\{1, \ldots, L\}$ for $i=0, \ldots, n-1$, which is contained in some open set $\mathcal{U} \subseteq \Lambda$ with the property that almost every point of $\mathcal{U}$ is recurrent for $f_{\mid \Lambda}$. Then, for every $\delta>0$ there exists an orbit $\left\{z_{i}\right\}_{i=0, \ldots, n}$ of $f$ in $M$, with $z_{i+1}=f^{k_{i}}\left(z_{i}\right)$ for some $k_{i}>0$, such that $d\left(z_{i}, x_{i}\right)<\delta$ for all $i=0, \ldots, n$.

Remark 3.8. In general situations, one has an abundance of homoclinic orbits. By the Smale-Birkhoff homoclinic orbit theorem the existence of a single transverse homoclinic orbit implies the existence of infinitely many transverse homoclinic orbits that are geometrically distinct. Thus one is able to define many scattering maps.

In applications, using several scattering maps rather than a single one can be very advantageous. In astrodynamics, for example, the existence of multiple homoclinic intersections can be exploited to obtain diffusion [43,47] and to increase the versatility of space missions; see, e.g., [19,40].

Remark 3.9. Using several scattering maps can also be useful to prove diffusion in generic systems. In some perturbative problems, e.g., as in Section 4 , the scattering map can be computed in terms of convergent integrals of the perturbation evaluated along a homoclinic of the unperturbed system. One can ensure that the scattering map has nontrivial effects by verifying that such an integral is nonzero. Thus, given a perturbation, one can slightly modify it, using a bump function supported in some tubular neighborhood of the homoclinic, to obtain a nearby perturbation for which the corresponding scattering map exhibits the desired nontrivial effects. Having available multiple homoclinics, one can use bump functions supported in disjoint 
tubular neighborhoods of each of these homoclinics to obtain multiple scattering maps that exhibit different types of nontrivial behaviors; see, e.g., [22, 23, 55, 56].

Remark 3.10. The results above also generalize to the case of several NHIMs. If

$$
\Gamma_{1,2} \subseteq W_{\Lambda_{1}}^{u} \cap W_{\Lambda_{2}}^{s}
$$

is a heteroclinic channel between two NHIMs $\Lambda_{1}$ and $\Lambda_{2}$, we can define a scattering map

$$
\sigma_{1,2}: \Omega^{-}\left(\Gamma_{1,2}\right) \subseteq \Lambda_{1} \rightarrow \Lambda_{2}
$$

in a similar fashion to the case of a single NHIM. If we are given a chain of manifolds $\Lambda_{i}, i=1, \ldots, n$, and scattering maps

$$
\sigma_{i, i+1}: \Omega^{-}\left(\Gamma_{i, i+1}\right) \subseteq \Lambda_{i} \rightarrow \Lambda_{i+1}, \quad i=1, \ldots, n-1,
$$

then we can shadow orbits of the form $y_{i+1}=\sigma_{i, i+1}\left(y_{i}\right)$, with $y_{i} \in \Lambda_{i}$ and $y_{i+1} \in \Lambda_{i+1}$, for $i=1, \ldots, n-1$. Such scattering maps appear in the study of double resonances [6, 68, 69,77]. We hope to come back to this problem.

Another problem where one has scattering maps between two different normally hyperbolic invariant manifolds is the problem of two rocking blocks under periodic forcing [62].

\subsection{A Qualitative Mechanism of Diffusion in a Perturbative Symplectic Setting}

We now describe several situations when we can construct pseudo-orbits of the scattering map that travel a significant distance within the normally hyperbolic invariant manifold, and so Theorem 3.6 can be applied to obtain true orbits nearby. More concrete conditions that yield such orbits in some concrete examples appear in Section 4

We consider the perturbative setting described in Section 2.2, where $f_{\varepsilon}: M \rightarrow$ $M$ is a symplectic map, $\Lambda_{\varepsilon} \subseteq M$ is a normally hyperbolic invariant manifold (not necessarily compact) for $f_{\varepsilon}, \Gamma_{\varepsilon}$ is a homoclinic channel for $f_{\varepsilon}$, and $\sigma_{\varepsilon}: \Omega^{-}\left(\Gamma_{\varepsilon}\right) \rightarrow$ $\Omega^{+}\left(\Gamma_{\varepsilon}\right)$ is the corresponding scattering map for $\varepsilon \in\left(\varepsilon_{0}, \varepsilon_{0}\right)$. We assume that $\Lambda_{\varepsilon}$ is described via a symplectic parametrization $k_{\varepsilon}: \Lambda_{0} \rightarrow \Lambda_{\varepsilon}$, and let $\left(\widetilde{f_{\varepsilon}}\right)_{\mid \Lambda_{0}}=$ $k_{\varepsilon}^{-1} \circ\left(f_{\varepsilon}\right)_{\mid \Lambda_{\varepsilon}} \circ k_{\varepsilon}, \widetilde{\sigma}_{\varepsilon}=k_{\varepsilon}^{-1} \circ \sigma_{\varepsilon} \circ k_{\varepsilon}$.

Below, in Theorem 3.11, we will use the perturbative formula for the scattering map (2.3) with $\widetilde{\sigma}_{0}=\mathrm{Id}$, and with a slightly more general first-order perturbation term of the scattering map. This allows us to apply the result of Theorem 3.11 to more degenerate cases, where second-order perturbation theory is necessary to detect the transversality between the stable and unstable manifolds, or to the socalled "a priori stable" case, where the Melnikov potential can be exponentially small in $\varepsilon$; see Remark 3.16 .

THEOREM 3.11. Assume that for all $\varepsilon \in\left(-\varepsilon_{0}, \varepsilon_{0}\right)$, there exists a scattering map $\sigma_{\varepsilon}$, defined in a domain $U:=k_{\varepsilon}^{-1}\left(\Omega^{-}\left(\Gamma_{\varepsilon}\right)\right) \subset \Lambda_{0}$, such that

$$
\tilde{\sigma}_{\varepsilon}=\mathrm{Id}+\mu(\varepsilon) J \nabla S+g(\mu(\varepsilon)), \quad g(\mu(\varepsilon))=o(\mu(\varepsilon)),
$$


where $S$ is some real-valued $C^{\ell}$-function on $U \subset \Lambda_{0}$, and $g(\mu(\varepsilon))$ and $\mu(\varepsilon)$ are some $C^{\ell}$-functions, being defined on $\left(-\varepsilon_{0}, \varepsilon_{0}\right)$ with $\mu(0)=0$; by $g(\mu(\varepsilon))=$ $o(\mu(\varepsilon))$ we mean that $\lim _{\varepsilon \rightarrow 0} g(\mu(\varepsilon)) / \mu(\varepsilon)=0$.

Suppose that

$$
J \nabla S\left(\tilde{x}_{0}\right) \neq 0 \text { at some point } \tilde{x}_{0} \in U \subset \Lambda_{0} .
$$

Let $\tilde{\gamma}:[0,1] \rightarrow \Lambda_{0}$ be an integral curve through $\tilde{x}_{0}$ for the vector field $J \nabla S$. Suppose that there exists a neighborhood $\mathcal{U}_{\tilde{\gamma}} \subset U$ of $\tilde{\gamma}([0,1])$ in $\Lambda_{0}$ such that a.e. point in $\mathcal{U}_{\tilde{\gamma}}$ is recurrent for $\tilde{f}_{\varepsilon \mid \Lambda_{0}}$. Let $\gamma_{\varepsilon}=k_{\varepsilon} \circ \tilde{\gamma}$ be the corresponding curve in $\Lambda_{\varepsilon}$.

There exists $\varepsilon_{1}>0$ sufficiently small and a constant $K>0$ such that for every $\varepsilon \in\left(-\varepsilon_{1}, \varepsilon_{1}\right), \varepsilon \neq 0$, and every $\delta>0$, there exists an orbit $\left\{z_{i}\right\}_{i=0, \ldots, n}$ of $f_{\varepsilon}$ in $M$, with $n=O\left(\mu(\varepsilon)^{-1}\right)$, such that for all $i=0, \ldots, n-1$,

$$
z_{i+1}=f_{\varepsilon}^{k_{i}}\left(z_{i}\right) \text { for some } k_{i}>0
$$

and for all $i=0, \ldots, n$, we have

$$
d\left(z_{i}, \gamma_{\varepsilon}\left(t_{i}\right)\right)<\delta+K(\mu(\varepsilon)+|g(\mu(\varepsilon)) / \mu(\varepsilon)|) \quad \text { for } t_{i}=i \cdot \mu(\varepsilon),
$$

where $0=t_{0}<\cdots<t_{n} \leq 1$.

The proof of this theorem is given in Section 5.3 .

We will refer to a solution curve $\tilde{\gamma}$ in $\Lambda_{0}$ as in the statement of Theorem 3.11, or to its corresponding curve $\gamma_{\varepsilon}=k_{\varepsilon}(\tilde{\gamma})$ in $\Lambda_{\varepsilon}$, as a "scattering path," as it represents an approximation of an orbit of the scattering map; see Figure 5.3. So the previous result can be stated that, given any scattering path, there exits a true orbit of the system that shadows it.

In applications, it is often the case that $\Lambda_{0}=B^{d} \times \mathbb{T}^{d}$, and we have a system of action-angle coordinates $(I, \phi)$ on $\Lambda_{0}$ with $I \in B^{d}$ and $\phi \in \mathbb{T}^{d}$, where $\mathbb{T}^{d}=$ $\mathbb{R}^{d} / \mathbb{Z}^{d}$ and $B^{d} \subseteq \mathbb{R}^{d}$ is a disk in $\mathbb{R}^{d}$ or $B^{d}=\mathbb{R}^{d}$. Since one can typically find a scattering path for which the action variable changes by some positive distance independent of $\varepsilon$, implicitly one can find a true orbit for which the action variable changes by $O(1)$; this is stated precisely in the following corollary.

There exists a sufficiently small neighborhood $V_{\Lambda_{\varepsilon}}$ of $\Lambda_{\varepsilon}$ in $M$ such that for every point $z \in V_{\Lambda_{\varepsilon}}$ there exists a unique point $z^{\prime} \in \Lambda_{\varepsilon}$ that is the closest point to $z$. The point $z^{\prime}$ is the image of some unique point $\tilde{z} \in \Lambda_{0}$ via $k_{\varepsilon}$, i.e., $z^{\prime}=$ $k_{\varepsilon}(\tilde{z})$. We denote by $I(z)$ the $I$-coordinate of the corresponding point $\tilde{z} \in \Lambda_{0}$, i.e., $I(z):=I(\widetilde{z})$.

COROLlary 3.12. Assume that a scattering map $\sigma_{\varepsilon}$ as in Theorem 3.11 is given. If $J \nabla S$ is transverse to some level set $\left\{I=I_{*}\right\}$ in $\Lambda_{0}$ at some point $\left(I_{*}, \phi_{*}\right) \subset U$, then there exist $0<\varepsilon_{1}<\varepsilon_{0}$ and $\rho>0$ such that for every $0<\varepsilon<\varepsilon_{1}$ there exists an orbit $\left\{z_{i}\right\}_{i=0, \ldots, n}$ of $f_{\varepsilon}$ such that

$$
\left\|I\left(z_{n}\right)-I\left(z_{0}\right)\right\|>\rho .
$$


The proof of Corollary 3.12 is given in Section 5.4

Remark 3.13. The key assumptions for Theorem 3.11 are the existence of a normally hyperbolic invariant manifold, the recurrence property of the inner dynamics, and the existence of transverse homoclinic intersections yielding scattering maps. In the perturbative setting that we consider, the existence of a normally hyperbolic invariant manifold for some range of $\varepsilon$ 's follows from the standard theory of normally hyperbolic invariant manifolds; see, e.g., [49, 66]. The recurrence property of the inner dynamics is automatically satisfied in the symplectic setting that we consider. The transversality conditions (A.6) and A.7) are necessary to ensure by our method the existence of a scattering map (of course, there may be other methods).

Since these conditions play an important role, let us discuss somewhat informally their abundance in the space of dynamical systems. Both conditions (A.6) and (A.7) are transversality conditions. The existence of manifolds satisfying both of them is a $C^{1}$-open condition.

Of course, like all transversality conditions, A.6 and A.7) can fail in a persistent way. There are examples where the transversality is lost in a codimension 1 manifold in such a way that all the perturbations will still have another such manifold. (Intuitively, transversality amounts to some determinant of a matrix of derivatives being away from 0 . If it happens to change sign in some neighborhood, all perturbations will have some sign change). Note, however, that small perturbations can create the desired transversality at one point.

The condition A.6 has been very well studied in dynamical systems. If it holds, we have also infinitely many other intersections satisfying (A.6) obtained by iteration, and under mild conditions there are also secondary intersections. It could very well be that the secondary intersections satisfy A.6 in some appreciable region.

It is important to remark that the application of the results only requires the existence of one channel satisfying the two conditions.

Let us mention that in many cases of practical interest, when the existence of transverse homoclinic intersections can be established via Melnikov theory (including systems with small dissipation, as in [55]), the conditions (A.6) and (A.7) are generically satisfied on open sets of order $O(1)$. This is also the case for the a priori unstable, nearly integrable Hamiltonian systems considered in Section 4. in which case the existence of a homoclinic manifold satisfying (A.6) and A.7) is a generic condition; i.e., it is satisfied by a $C^{1}$-open and $C^{\infty}$-dense set of perturbations. In particular, the homoclinic manifold turns out to be a graph over the center variables. Moreover, for this class of Hamiltonian systems, the condition (3.3) on the corresponding scattering map, which ensures the existence of orbits that travel $O(1)$, is generically satisfied.

We shall also mention that there are some special classes of systems (symplectic, volume preserving) for which the existence of homoclinics always holds (see, e.g., [41, 46, 72, 87, 93]). 
Remark 3.14. Let us note that the diffusion orbit $\left\{z_{i}\right\}_{i=0, \ldots, n}$ obtained in Corollary 3.12 does not necessarily follow a given pseudo-orbit of the scattering map. If the dynamics given by $f_{\varepsilon}$ has diffusing orbits, these are the ones obtained in the corollary. In case the dynamics of $f_{\varepsilon}$ remains in a bounded set, we need to follow the pseudo-orbits of the scattering map $\sigma_{\varepsilon}$ to obtain the diffusing ones.

Besides this corollary, later in Section 4, where we will have more details of the construction of the perturbed normally hyperbolic manifold $\Lambda_{\varepsilon}$, we will be able to prove that one can always follow any orbit of the scattering map or combinations of orbits of several scattering maps while they stay in a compact subset of $\Lambda_{\varepsilon}$. This will allow us to obtain stronger results on diffusion to travel predesigned itineraries in the actions.

Remark 3.15. We note that, in order to obtain a trajectory that achieves a change in the $I$-variable of order $O(1)$, the scattering map needs to be applied $n=$ $O\left(\mu(\varepsilon)^{-1}\right)$ times. However, the true orbit that achieves the $O(1)$-change in the $I$-variable follows not only the scattering map but also some recursive orbit segments of the inner dynamics, as in the proof of Theorem 3.6. Since these recursive orbit segments of the inner dynamics are obtained by invoking the Poincaré recurrence theorem, the above result does not yield an estimate for the time required to follow the inner dynamics, and hence does not directly lead to an estimate on the diffusion time.

Remark 3.16. The condition that the unperturbed scattering map is the identity, i.e., $\widetilde{\sigma}_{0}=\mathrm{Id}$, is naturally satisfied in some examples, e.g., in the a priori unstable system in Section 4. The function $\mu(\varepsilon)$ is associated to the size of the splitting of $W^{u}\left(\Lambda_{\varepsilon}\right)$ and $W^{s}\left(\Lambda_{\varepsilon}\right)$.

In the example in Section 4, we have $\mu(\varepsilon)=\varepsilon$ and $g(\mu(\varepsilon))=O\left(\varepsilon^{2}\right)$ in the generic case. Nevertheless, in some degenerate cases, it can happen that, up to first order in $\varepsilon$, the perturbed stable and unstable invariant manifolds of $\Lambda_{\varepsilon}$ coincide. In these cases it is necessary to go to second-order perturbation theory to distinguish them and therefore $\mu(\varepsilon)=\varepsilon^{2}$ and $S$ in $(2.5)$ has a different expression (not given here) in terms of the second-order variationals along the unperturbed homoclinic orbit.

Another special situation occurs in the so-called a priori stable systems, where the unperturbed system is completely integrable without any hyperbolic structure. In those cases, the a priori unstable structure appears after some first-order partial averaging near simple resonances, giving rise to a system of the form $\widetilde{H}_{\varepsilon}^{0}+\widetilde{H}_{\varepsilon}^{1}$. Therefore the analogue of the unperturbed homoclinic orbit $\phi_{t}(z)$, which appears in the formulas of the scattering map (2.5), is $\varepsilon$-dependent, i.e., $\phi_{t, \varepsilon}(z)$. The splitting between the stable and unstable manifolds behaves differently from the a priori unstable case with respect to the perturbation parameter. Concretely, we have:

$$
\tilde{\sigma}_{\varepsilon}=\mathrm{Id}+J \nabla S+g(\mu(\varepsilon)),
$$


where

$$
\begin{aligned}
S(x, \varepsilon)= & \lim _{T \rightarrow+\infty} \int_{-T}^{0}\left[\widetilde{H}_{\varepsilon}^{1} \circ \phi_{t, \varepsilon}(z)-\widetilde{H}_{\varepsilon}^{1} \circ \phi_{t, \varepsilon}(x)\right] d t \\
& +\lim _{T \rightarrow+\infty} \int_{0}^{T}\left[\widetilde{H}_{\varepsilon}^{1} \circ \phi_{t, \varepsilon}(z)-\widetilde{H}_{\varepsilon}^{1} \circ \phi_{t, \varepsilon}(x)\right] d t .
\end{aligned}
$$

and $S$ and $g$ satisfy

$$
S(x, \varepsilon)=O(\mu(\varepsilon)), \quad g(\mu(\varepsilon))=o(\mu(\varepsilon)) .
$$

If the system is analytic, there is an exponentially small splitting of the separatrices and therefore $\mu(\varepsilon)=O\left(\varepsilon^{p} \exp \left(-q \varepsilon^{-r}\right)\right)$ for some $p, q, r \in \mathbb{Q}$, as in [2]. Nevertheless, to obtain the behavior of the error function $g(\mu(\varepsilon))$ in general analytic a priori stable systems is still an open and difficult question. If the system is only smooth, one usually has $\mu(\varepsilon)=\varepsilon^{p}$ for $p \geq 2$.

Besides the above comments, we want to stress that, once a formula like $(3.2)$ is established, the results of Theorem 3.11 remain true.

\section{Existence of Diffusing Trajectories in Nearly Integrable A Priori Unstable Hamiltonian Systems}

As an application, we show the existence of diffusing orbits in a large class of nearly integrable a priori unstable Hamiltonian systems that are multi-dimensional both in the center and in the hyperbolic directions. The model below is an extension of those considered in [34, 37, 42].

Let

$$
\begin{aligned}
H_{\varepsilon}(p, q, I, \phi, t)= & h_{0}(I)+\sum_{i=1}^{n} \pm\left(\frac{1}{2} p_{i}^{2}+V_{i}\left(q_{i}\right)\right) \\
& +\varepsilon H_{1}(p, q, I, \phi, t ; \varepsilon) .
\end{aligned}
$$

where $(p, q, I, \phi, t) \in \mathbb{R}^{n} \times \mathbb{T}^{n} \times \mathbb{R}^{d} \times \mathbb{T}^{d} \times \mathbb{T}^{1}$.

We make the following assumptions:

(A1) The functions $h_{0}, H_{1}$, and $V_{i}, i=1, \ldots, n$, are uniformly $C^{r}$ for $r \geq r_{0}$.

(A2) Each potential $V_{i}: \mathbb{T}^{n} \rightarrow \mathbb{R}, i=1, \ldots, n$, is 1 -periodic in $q_{i}$ and has a nondegenerate (in the sense of Morse) global maximum at 0 ; hence each "pendulum" $\pm\left(\frac{1}{2} p_{i}^{2}+V_{i}\left(q_{i}\right)\right)$ has a homoclinic orbit to $(0,0)$, parametrized by $\left(p_{i}^{0}(t), q_{i}^{0}(t)\right), t \in \mathbb{R}$.

To formulate the next assumption (A3), which has two parts (A3.a) and (A3.b), we need to introduce some other tools.

- Let $\tilde{\Lambda}_{0}=\{(p, q, I, \phi, t) \mid p=q=0\}$. By (A2) there is a family of homoclinic orbits for the whole system of pendula given by

$$
\begin{aligned}
\left(p^{0}(\tau+t \overline{1}), q^{0}(\tau+t \overline{1})\right) & = \\
& \left(p_{1}^{0}\left(\tau_{1}+t\right), \ldots, p_{n}^{0}\left(\tau_{n}+t\right), q_{1}^{0}\left(\tau_{1}+t\right), \ldots, q_{n}^{0}\left(\tau_{n}+t\right)\right),
\end{aligned}
$$


where $\tau=\left(\tau_{1}, \ldots, \tau_{n}\right) \in \mathbb{R}^{n}$ and $\overline{1}=(1, \ldots, 1) \in \mathbb{R}^{n}$.

- Let $\widetilde{\Gamma}_{0} \subseteq\left\{\left(p^{0}(\tau), q^{0}(\tau), I, \phi, t\right) \mid \tau \in \mathbb{R}^{n}, I \in \mathbb{R}^{d}, \phi \in \mathbb{T}^{d}, t \in \mathbb{T}^{1}\right\}$ be a homoclinic channel for which we can define a scattering map $\widetilde{\sigma}_{0}$ on $\widetilde{\Lambda}_{0}$.

- Let the Poincaré function (or Melnikov potential) associated to the homoclinic manifold $\widetilde{\Gamma}_{0}$ be:

$$
\begin{gathered}
L(\tau, I, \phi, s) \\
=-\int_{-\infty}^{\infty}\left[H_{1}\left(p^{0}(\tau+t \overline{1}), q^{0}(\tau+t \overline{1}), I, \phi+\omega(I) t, s+t ; 0\right)\right. \\
\left.H_{1}(0,0, I, \phi+\omega(I) t, s+t ; 0)\right] d t .
\end{gathered}
$$

where $\omega(I)=\partial h_{0} / \partial I$.

The first part of assumption (A3) is the following:

(A3.a) The perturbation $H_{1}$ is 1-periodic in $t$ and satisfies some explicit nondegeneracy conditions as described below. Assume that there exists a set $U^{-}:=\mathcal{I} \times \mathcal{J} \subset \mathbb{R}^{d} \times \mathbb{T}^{d+1}$ such that $\mathcal{I}$ is an open ball in $\mathbb{R}^{d}$, and for any values $(I, \phi, s) \in U^{-}$, the map

$$
\tau \in \mathbb{R}^{n} \rightarrow L(\tau, I, \phi, s) \in \mathbb{R}
$$

has a nondegenerate critical point $\tau^{*}$, which is locally given, by the implicit function theorem, by

$$
\tau^{*}=\tau^{*}(I, \phi, s) \text {. }
$$

To formulate the next assumption we need to introduce some other tools.

- Define the auxiliary functions

$$
\mathcal{L}(I, \phi, s)=L\left(\tau^{*}(I, \phi, s), I, \phi, s\right), \quad \mathcal{L}^{*}(I, \theta)=\mathcal{L}(I, \theta, 0) .
$$

We regard $\mathcal{L}^{*}(I, \theta)$ as a function on the set

$$
\operatorname{Dom}\left(\mathcal{L}^{*}\right)=\left\{(I, \theta) \in \mathbb{R}^{d} \times \mathbb{T}^{d} \mid \exists s \in \mathbb{T}^{1} \text { s.t. }(I, \theta+\omega(I) s, s) \in U^{-}\right\} .
$$

The second part of assumption (A3) is:

(A3.b) Assume that the reduced Poincare function $\mathcal{L}^{*}(I, \theta)$ satisfies that $J \nabla \mathcal{L}^{*}(I, \theta)$ is transverse, relative to $\mathbb{R}^{d} \times \mathbb{T}^{d}$, to the level set $\left\{I=I_{*}\right\}$ at some point $\left(I_{*}, \theta_{*}\right)=\left(I_{*}, \phi_{*}-\omega\left(I_{*}\right) s\right)$, with $\left(I_{*}, \phi_{*}, s\right) \in U^{-}$. That is,

$$
\frac{\partial \mathcal{L}^{*}}{\partial \theta}\left(I_{*}, \theta_{*}\right) \neq 0 \text {. }
$$

We note that the integral in (4.2) is similar to that in (2.4) and (2.5), as it concerns the average effect of the perturbation $H_{1}$ on a homoclinic orbit of the unperturbed system.

The result below states that, for all small enough regular perturbations satisfying (4.4), there exist trajectories that travel $O(1)$ with respect to the $I$-coordinate; that is, they travel a distance relative to the $I$-coordinate that is independent of the size of the perturbation. This phenomenon is referred to as Arnold diffusion. 
THEOREM 4.1. Assuming conditions (A1)-(A3), there exists $\varepsilon_{0}>0$ and $\rho>0$ such that, for each $\varepsilon \in\left(0, \varepsilon_{0}\right)$, there exists a trajectory $x(t)$ of the Hamiltonian flow of Hamiltonian (4.1) and $T>0$ such that

$$
\|I(x(T))-I(x(0))\|>\rho .
$$

Remark 4.2. We emphasize some advantages of Theorem 4.1 in comparison to the main results of [34, 37, 42, 60]:

- Both the phase space of $h_{0}$ and that of the system of pendula are multidimensional.

- We do not assume a convexity condition on the unperturbed Hamiltonian $H_{0}(I, \phi, p, q)=h_{0}(I)+\sum_{i=1, \ldots, n} \pm\left(p_{i}^{2} / 2+V_{i}\left(q_{i}\right)\right)$, which is typically required when using variational methods.

- We do not assume that $h_{0}$ satisfies a nondegeneracy condition that $I \mapsto$ $\partial h_{0} / \partial I$ is a diffeomorphism, or a convexity condition that $\partial^{2} h_{0} / \partial I_{i} \partial I_{j}$ is strictly positive/negative definite. In the lack of such conditions, one cannot apply the KAM theorem, and hence cannot construct transition chains of KAM tori. Also, Aubry-Mather theory cannot be applied.

- We do not assume that $H_{1}$ is a trigonometric polynomial. Moreover, we note that condition (A3) is satisfied by a $C^{r}$ open and dense set of perturbations $H_{1}$.

In the method of [37] one needs to check a different condition (which is clearly generic) around every first-order resonance. In concrete systems, when one is interested in a practical problem (e.g., in the three-body problem) and not in generic statements, the verification of the mechanism of [37] is possible, albeit tedious. With the present method, the verification in concrete systems of interest is much more straightforward; see, e.g., [19].

From now on, we use the following notation: When we say that some error term is bounded by a constant, or by $O\left(\varepsilon^{a}\right)$, or by $O\left(\varepsilon^{a} \ln \left(\varepsilon^{b}\right)\right)$, we mean uniformly on some compact set.

Proof of THEOREM 4.1. We describe the geometric structures that organize the dynamics, following [34, 37]. We emphasize that, once the geometric setup is laid out, the dynamics argument to show the existence of diffusing orbits is very different.

The time-dependent Hamiltonian in (4.1) is transformed into an autonomous Hamiltonian by introducing a new variable $A$, symplectically conjugate with $t$, obtaining the $(n+d+1)$-degrees-of-freedom Hamiltonian system

$$
\begin{aligned}
\widetilde{H}_{\varepsilon}(p, q, I, \phi, A, t)= & h_{0}(I)+\sum_{i=1}^{n} \pm\left(\frac{1}{2} p_{i}^{2}+V_{i}\left(q_{i}\right)\right)+A \\
& +\varepsilon H_{1}(p, q, I, \phi, t ; \varepsilon) .
\end{aligned}
$$


The variable $A$ does not play any dynamical role, as it does not appear in any of the Hamiltonian equations for any of the variables, including itself.

With an abuse of notation, we denote

$$
\tilde{\Lambda}_{0}:=\left\{(p, q, I, \phi, A, t) \mid p=q=0, I \in \mathcal{I}, A \in \mathbb{R},(\phi, t) \in \mathbb{T}^{d+1}\right\} .
$$

This is a normally hyperbolic invariant manifold for the extended Hamiltonian flow; $\tilde{\Lambda}_{0}$ is diffeomorphic to $\left(\mathbb{R}^{d} \times \mathbb{T}^{d}\right) \times(\mathbb{R} \times \mathbb{T})$.

We fix an energy manifold $\left\{\widetilde{H}_{\varepsilon}=\widetilde{h}\right\}$ for some $\tilde{h}$, and restrict to a Poincaré section $\{t=s\}$ for the Hamiltonian flow. The resulting manifold is a $(2 n+2 d)$ dimensional manifold, which we denote by $M_{\varepsilon}$. The first return map to $M_{\varepsilon}$ of the Hamiltonian flow is a $C^{r}$-differentiable map denoted $f_{\epsilon}$.

The manifold

$$
\Lambda_{0}:=\left\{(p, q, I, \phi) \mid p=q=0, I \in \mathcal{I}, \phi \in \mathbb{T}^{d}\right\} \subseteq M_{0}
$$

is a normally hyperbolic invariant manifold for $f_{0}$, which is independent of the section $\{t=s\}$. Note that $\Lambda_{0}$ is diffeomorphic to $\mathbb{R}^{d} \times \mathbb{T}^{d}$.

Thus, both $\tilde{\Lambda}_{0}$ and $\Lambda_{0}$ are noncompact.

Note that the restriction of $f_{0}$ to $\Lambda_{0}$ is an integrable map, as $f_{0}(0,0, I, \phi)=$ $(0,0, I, \phi+\omega(I))$, and $\Lambda_{0}$ is foliated by invariant $d$-dimensional tori given by $\{I=c t\}$.

Choose a closed ball $\bar{B}_{R}\left(I_{*}\right)$ in the action space $\mathbb{R}^{d}$ such that $J \nabla \mathcal{L}^{*}(I, \theta)$ is transverse, relative to $\operatorname{Dom}\left(\mathcal{L}^{*}\right)$, to each action level set $\left\{I=I_{0}\right\}$, which is an invariant torus, with $I_{0} \in \bar{B}_{R}\left(I_{*}\right)$. Choose $0<\rho<R$.

Denote

$$
\begin{aligned}
& \widetilde{\Lambda}_{0}^{\prime}:=\left\{(p, q, I, \phi, A, t) \mid p=q=0, I \in \bar{B}_{R}\left(I_{*}\right), A \in \mathbb{R},(\phi, t) \in \mathbb{T}^{d+1}\right\}, \\
& \Lambda_{0}^{\prime}:=\left\{(p, q, I, \phi) \mid p=q=0, I \in \bar{B}_{R}\left(I_{*}\right), \phi \in \mathbb{T}^{d}\right\},
\end{aligned}
$$

which are normally hyperbolic invariant manifolds with boundary for the flow and for the map, respectively, corresponding to $I \in \bar{B}_{R}\left(I_{*}\right)$.

Consider now the perturbed Hamiltonian system. Using a $C^{r}$-differentiable bump function we can modify the Hamiltonian $\widetilde{H}_{\varepsilon}$ to another Hamiltonian $\widetilde{\mathcal{H}}_{\varepsilon}$ that coincides with the original one for all $(p, q, I, \phi, A, t)$ with $I \in \bar{B}_{R}\left(I_{*}\right)$, and coincides with $H_{0}$ for all $(p, q, I, \phi, A, t)$ with $I$ outside of some open ball $B_{R^{\prime}}\left(I_{*}\right) \supseteq \bar{B}_{R}\left(I_{*}\right)$, with $R^{\prime}>R$. For all $\varepsilon$ sufficiently small, there exists a normally hyperbolic invariant manifold $\tilde{\Lambda}_{\varepsilon}$ for the flow of the modified Hamiltonian $\widetilde{\mathcal{H}}_{\varepsilon}$. The manifold $\widetilde{\Lambda}_{\varepsilon}$ is diffeomorphic to $\widetilde{\Lambda}_{0}$ via a $C^{\ell}$-smooth parametrization $\tilde{k}_{\varepsilon}: \tilde{\Lambda}_{0} \rightarrow \tilde{\Lambda}_{\varepsilon}$, with $\widetilde{k}_{0}=$ Id. Using this parametrization, we can describe $\tilde{\Lambda}_{\varepsilon}$ in terms of the coordinates $(I, \phi, A, t) \in \tilde{\Lambda}_{0}$. Similarly, there exists a $C^{\ell}$ smooth parametrization $k_{\varepsilon}: \Lambda_{0} \rightarrow \Lambda_{\varepsilon}$, with $k_{0}=\mathrm{Id}$, and we can then describe $\Lambda_{\varepsilon}$ in terms of the coordinates $(I, \phi) \in \Lambda_{0}$.

The manifold $\tilde{\Lambda}_{\varepsilon}$ is not unique, as it depends on the modified Hamiltonian vector field of $\widetilde{\mathcal{H}}_{\varepsilon}$, but what is important for us is that the extended Hamiltonian $\widetilde{\mathcal{H}}_{\varepsilon}$ 
coincides with $\widetilde{H}_{\varepsilon}$ at the points with $I \in \bar{B}_{R}\left(I_{*}\right)$. Therefore, if we find an orbit of $\widetilde{\mathcal{H}}_{\varepsilon}$ whose action $I$ stays in $\bar{B}_{R}\left(I_{*}\right)$, this orbit will also be a real orbit of $\widetilde{H}_{\varepsilon}$. Let us denote by $\widetilde{\phi}_{t, \varepsilon}^{\prime}$ the flow of $\widetilde{\mathcal{H}}_{\varepsilon}$, and by $f_{\varepsilon}^{\prime}$ the corresponding first return map.

Let

$$
\begin{aligned}
& \tilde{\Lambda}_{\varepsilon}^{\prime}:=\left\{\tilde{k}_{\varepsilon}(p, q, I, \phi, A, t) \mid p=q=0, I \in \bar{B}_{R}\left(I_{*}\right), A \in \mathbb{R},(\phi, t) \in \mathbb{T}^{d+1}\right\}, \\
& \Lambda_{\varepsilon}^{\prime}:=\left\{k_{\varepsilon}(p, q, I, \phi) \mid p=q=0, I \in \bar{B}_{R}\left(I_{*}\right), \phi \in \mathbb{T}^{d}\right\},
\end{aligned}
$$

be the normally hyperbolic manifolds for the perturbed flow and for the perturbed map, respectively, corresponding to $I \in \bar{B}_{R}\left(I_{*}\right)$. They are not invariant, but only locally invariant. The local invariance means, in the case of $\Lambda_{\varepsilon}^{\prime}$, that there exists a neighborhood $\mathcal{V}$ of $\Lambda_{\varepsilon}^{\prime}$ in $M_{\varepsilon}$ such that any orbit of $f_{\varepsilon}^{\prime}$ that stays in $\mathcal{V}$ for all time is actually contained in $\Lambda_{\varepsilon}^{\prime}$. The neighborhood $\mathcal{V}$ can be chosen independent of $\varepsilon$. The manifold $\Lambda_{\varepsilon}^{\prime}$ is compact and symplectic (see [36]).

Condition (A3) allows one to define a scattering map $\sigma_{\varepsilon}: \Omega^{-}\left(\Gamma_{\varepsilon}\right) \rightarrow \Omega^{+}\left(\Gamma_{\varepsilon}\right)$, with $\Omega^{-}\left(\Gamma_{\varepsilon}\right), \Omega^{+}\left(\Gamma_{\varepsilon}\right) \subseteq \Lambda_{\varepsilon}^{\prime}$. We will restrict to a homoclinic channel $\Gamma_{\varepsilon}$ that is compact.

As mentioned before, it is more convenient to express the scattering map $\sigma_{\varepsilon}$ as a map on $\Lambda_{0}$ via $\widetilde{\sigma}_{\varepsilon}=k_{\varepsilon}^{-1} \circ \sigma_{\varepsilon} \circ k_{\varepsilon}$. By hypothesis (A.3), we have $U^{-} \subseteq \operatorname{dom}\left(\widetilde{\sigma}_{\varepsilon}\right)=$ $k_{\varepsilon}^{-1}\left(\Omega^{-}\left(\Gamma_{\varepsilon}\right)\right)$. In a similar fashion, we consider $\tilde{f}_{\varepsilon}=k_{\varepsilon}^{-1} \circ f_{\varepsilon \mid \Lambda_{\varepsilon}} \circ k_{\varepsilon}$ on $\Lambda_{0}$.

The papers [36, 38, 53] show that condition (A3.a) implies that the scattering map can be expressed as

$$
\tilde{\sigma}_{\varepsilon}(I, \phi)=(I, \phi)+\varepsilon J \nabla \mathcal{L}^{*}(I, \phi-\omega(I) s)+O\left(\varepsilon^{2}\right),
$$

which is of the form (3.2) with $\mu(\varepsilon)=\varepsilon$ and $g(\mu(\varepsilon))=\varepsilon^{2}$. Of course, both the scattering map $\widetilde{\sigma}_{\varepsilon}$ and the Poincaré map $\tilde{f}_{\varepsilon}$ depend on the chosen section $\{t=s\}$.

The function $\mathcal{L}^{*}$ involved in condition (A3) plays the role of the function $S$ in Theorem 3.11 and Corollary 3.12. Condition 4.4 amounts to $J \nabla \mathcal{L}^{*}$ being transverse to one level set of the variable $I$. Therefore we can apply Corollary 3.12 for the normally hyperbolic invariant manifold $\Lambda_{\varepsilon}$ and the scattering map $\widetilde{\sigma}_{\varepsilon}$, obtaining an orbit $\left(z_{i}\right)_{i=0, \ldots, n}$ of $f_{\varepsilon}^{\prime}$ with $I\left(z_{0}\right)=I_{*}$ and $\left\|I\left(z_{n}\right)-I\left(z_{0}\right)\right\|>\rho$ for some $T>0$. Either the resulting orbit is so that the action $I$ along it stays in $\bar{B}_{R}\left(I_{*}\right)$ and hence this orbit is a real orbit of $f_{\varepsilon}$ along which the action changes by at least $\rho$, or the orbit is so that the action $I$ along it leaves $\bar{B}_{R}\left(I_{*}\right)$, in which case the action changes by at least $R>\rho$. In either case, we obtain a trajectory of $\widetilde{H}_{\mathcal{E}}$ along which the action $I$ changes by at least $\rho>0$, which is independent of $\varepsilon$.

Below, we consider the case when the Melnikov potential (4.2) has $l$ distinct nondegenerate critical points $\tau_{1}^{*}, \tau_{2}^{*}, \ldots, \tau_{l}^{*}$ of $L(\tau, I, \phi, s)$. Each critical point determines a scattering map $\sigma_{\varepsilon}^{i}$ for which there exists a corresponding scattering path $\gamma_{i}$ that is a solution curve of the Hamiltonian flows $\dot{x}=J \nabla \mathcal{L}_{i}^{*}(x)$ on $\Lambda_{\varepsilon}$, where $\mathcal{L}_{i}^{*}$ is defined by 4.3 for $i=1, \ldots, l$. Further, we assume that these scattering paths can be concatenated. The following result says we either have 
orbits that escape every compact set in $\Lambda_{\varepsilon}$, or we have orbits that follow any such prescribed sequence of paths.

COROLlary 4.3. Assume hypotheses (A1) and (A2) of Theorem 4.1 hold and that (A3) is satisfied for finitely many nondegenerate critical points of $L(\tau, I, \phi, s)$. Let $\sigma_{\varepsilon}^{i}: \Omega^{-}\left(\Gamma_{\varepsilon}^{i}\right) \rightarrow \Omega^{+}\left(\Gamma_{\varepsilon}^{i}\right)$ be the corresponding scattering maps, with $\Omega^{-}\left(\Gamma_{\varepsilon}^{i}\right)$, $\Omega^{+}\left(\Gamma_{\varepsilon}^{i}\right) \subseteq \Lambda_{\varepsilon}$, for $i=1, \ldots$, l. Suppose that $\Omega^{-}\left(\Gamma_{\varepsilon}^{i}\right) \cap \Omega^{-}\left(\Gamma_{\varepsilon}^{i+1}\right) \neq \varnothing$ for $i=1, \ldots, l-1$. Let $\mathcal{U}=\bigcup_{i=1, \ldots, l} \Omega^{-}\left(\Gamma_{\varepsilon}^{i}\right)$. Then, either

- for every compact set $\mathcal{K}$ in $\Lambda_{\varepsilon}$ containing $\mathcal{U}$, there exists $x_{0} \in \mathcal{U}$, an orbit $x(t)$ of the Hamiltonian $\widetilde{H}_{\mathcal{E}}$, and a time $T>0$ such that $x(0)=x_{0}$ and $x(T) \notin \mathcal{K}$

or

- for every sequence of scattering paths $\gamma_{\varepsilon}^{i}:\left[T_{i-1}, T_{i}\right] \rightarrow \Omega^{-}\left(\Gamma_{\varepsilon}^{i}\right)$, with $\gamma_{\varepsilon}^{i}\left(T_{i}\right)=\gamma_{\varepsilon}^{i+1}\left(T_{i}\right)$ for $i=1, \ldots, l-1$, and for every $\delta>0$, there exists an orbit $x(t)$ of the Hamiltonian $\widetilde{H}_{\varepsilon}$ and a time $T>0$ such that $x(0) \in B_{\delta}\left(\gamma_{\varepsilon}^{0}\left(T_{0}\right)\right)$ and $x(T) \in B_{\delta}\left(\gamma_{\varepsilon}^{l}\left(T_{l}\right)\right)$.

Papers where the authors combine several scattering maps to obtain scattering paths are, for instance [44, 45], where this corollary can be applied, and also [43] in a different setting.

Remark 4.4. For the above results, we do not require the nondegeneracy condition that $I \mapsto \omega(I)=\partial h_{0} / \partial I$ is a diffeomorphism. Note that in the case when $d=1$ such a nondegeneracy condition implies that $\tilde{f}_{\varepsilon}$ is a monotone twist map relative to the $(I, \phi)$ coordinates. In our case, we allow $\tilde{f}_{\varepsilon}$ to be a nontwist map, which happens, for instance, if $h_{0}(I)=I^{n}$ with $n \geq 3$ odd. It is well-known that nontwist maps arise in many concrete models, e.g., in magnetic fields of toroidal plasma devices (such as tokamaks, which have reversed magnetic shear), models of transport by traveling waves in shear flows with zonal flow, and models of satellite orbits near critical inclination. Unlike twist maps, nontwist maps have regions where the KAM theorem and the Aubry-Mather theory do not apply; see [30, 31] and the references listed therein.

\section{Proofs of the Main Results}

\subsection{Proof of Lemma 3.1}

We provide two proofs of Lemma 3.1 .

The first proof uses the topological method of correctly aligned windows (see Appendix C) and is constructive in the sense that it provides an explicit algorithm to detect orbits with prescribed itineraries. It can also be used to provide quantitative estimates for the diffusion time (see Remark 3.3 and Remark 5.5).

The second proof uses the obstruction argument and is shorter. 


\subsubsection{A Proof Using Correctly Aligned Windows}

5.1.1.A Outline. We will construct windows that are correctly aligned and utilize them in two different ways: first, to define the integers $n^{*}$ and $m_{i}^{*}$ that appear in the statement of the lemma, and second, to show that, for a given pseudo-orbit as in the statement of the lemma, there exists a true orbit that shadows it.

For the first part, starting with a homoclinic point, we define a pair of "prototype" windows that are correctly aligned, with one window in a neighborhood of some negative iterate of the homoclinic point, and another window in a neighborhood of some positive iterate of the homoclinic point. There are conditions on the number of such iterates that provide us with the integer $n^{*}$. Then we consider a second homoclinic point and construct a second pair of "prototype" windows, in a similar fashion. To make the second window from the first pair correctly aligned with the first window from the second pair, we need to apply a sufficiently large number of iterates that is no less than some integer $m^{*}$. When this construction is repeated $i$ times, it provides us with an integer $m_{i}^{*}$ that depends on all previous windows.

For the second part, there is given a pseudo-orbit generated by alternatively applying the scattering map and the inner dynamics; the orders of the iterates of the inner map are required to satisfy conditions that depend on the integers $n^{*}$ and

$m_{i}^{*}$. Then, the above-mentioned windows can be used to construct a sequence of correctly aligned windows along the pseudo-orbit. The existence of an orbit that follows these windows, and, in particular, shadows the given pseudo-orbit, follows from the shadowing property of correctly aligned windows (Theorem C.4).

We proceed in several steps.

5.1.1.B Choice of balls. We choose a system of linearized coordinates (see Appendix (B), given by $h: U_{\Lambda} \rightarrow V_{\Lambda}$, so that $V_{\Lambda}$ is contained in a $\delta$-neighborhood of $\Lambda$.

By the compactness of $\Lambda$ or the uniform regularity of $f$, there exists $\delta_{1}>0$ such that, whenever $x_{c} \in \Lambda,\left\|v_{u}\right\|,\left\|v_{s}\right\|<\delta_{1}$, and $0<\rho_{c}, \rho_{u}, \rho_{s}<\delta_{1}$, the image of

$$
B_{\rho_{c}}\left(x_{c}\right) \times B_{\rho_{u}}\left(v_{u}\right) \times B_{\rho_{s}}\left(v_{s}\right) \subseteq U_{\Lambda}
$$

under $h$ is contained in $V_{\Lambda}$ and has diameter less than $\delta / 2$.

We choose and fix $\rho_{c}, \rho_{u}, \rho_{s}$ as in 5.1.

By the normal hyperbolicity of $\Lambda$, there exist $0<\lambda_{-}<\lambda_{+}<\mu_{+}^{-1}<\mu_{-}^{-1}<1$ such that for each pair of balls $B_{\rho_{u}}\left(v_{u}\right) \subseteq E_{x}^{u}$ and $B_{\rho_{s}}\left(v_{s}\right) \subseteq E_{x}^{s}$, with $x \in \Lambda$, we have

$$
\begin{aligned}
B_{\rho_{u} \mu_{-}}\left(D f_{\mid E^{u}}\left(v_{u}\right)\right) \subseteq D f_{\mid E^{u}}\left(B_{\rho_{u}}\left(v_{u}\right)\right) \subseteq B_{\rho_{u} \mu_{+}}\left(D f_{\mid E^{u}}\left(v_{u}\right)\right), \\
B_{\rho_{s} \lambda_{-}}\left(D f_{\mid E^{s}}\left(v_{s}\right)\right) \subseteq D f_{\mid E^{s}}\left(B_{\rho_{s}}\left(v_{s}\right)\right) \subseteq B_{\rho_{s} \lambda_{+}}\left(D f_{\mid E^{s}}\left(v_{s}\right)\right) .
\end{aligned}
$$


5.1.1.C Lambda lemma. Consider a homoclinic channel $\Gamma$ and the corresponding scattering map $\sigma:=\sigma^{\Gamma}: \Omega^{-}(\Gamma) \rightarrow \Omega^{+}(\Gamma)$.

Let $p \in \Gamma$ and let $p^{-}, p^{+} \in \Lambda$ be the unique points for which $W^{u}\left(p^{-}\right) \cap$ $W^{s}\left(p^{+}\right) \cap \Gamma=\{p\}$. For given $k^{-}, k^{+}$, denote:

$$
\begin{aligned}
f^{-k^{-}}\left(p^{-}\right) & =h\left(x_{c}^{-}, 0,0\right), \quad f^{-k^{-}}(p)=h\left(x_{c}^{-}, v_{u}^{-}, 0\right), \\
f^{k^{+}}\left(p^{+}\right) & =h\left(x_{c}^{+}, 0,0\right), \quad f^{k^{+}}(p)=h\left(x_{c}^{+}, 0, v_{s}^{+}\right) .
\end{aligned}
$$

Due to the compactness of $\Gamma$ and the exponential contraction of the unstable (stable) fibers under negative (positive) iterates of $f$, there exists $n^{*}>0$ sufficiently large such that for every $k^{-} \geq n^{*}$ and $k^{+} \geq n^{*}$ we have the following:

(i) The point $f^{-k^{-}}(p) \in W^{u}\left(f^{-k^{-}}\left(p^{-}\right)\right)$satisfies $\left\|v_{u}^{-}\right\|<\rho_{u}<\delta_{1}$. This implies $f^{-k^{-}}(p) \in V_{\Lambda}$ and is $(\delta / 2)$-close to $f^{-k^{-}}\left(p^{-}\right)$.

(ii) The point $f^{k^{+}}(p) \in W^{s}\left(f^{k^{+}}\left(p^{+}\right)\right)$satisfies $\left\|v_{s}^{+}\right\|<\rho_{s}<\delta_{1}$. This implies $f^{k^{+}}(p) \in V_{\Lambda}$ and is $(\delta / 2)$-close to $f^{k^{+}}\left(p^{+}\right)$.

Since $\Gamma$ is an homoclinic channel, $W^{s}\left(p^{+}\right)$is transverse to $W^{u}(\Lambda)$ at $p$, and $W^{u}\left(p^{-}\right)$is transverse to $W^{s}(\Lambda)$ at $p$.

We apply two versions of the lambda lemma [26, 27, 50, 65, 85], and derive two transversality properties. The first version is concerned with the asymptotic behavior of the backwards iterates of an $\left(n_{s}\right)$-dimensional manifold transverse to $W^{u}(\Lambda)$. The second version is concerned with the asymptotic behavior of the forward iterates of an $\left(n_{u}+n_{c}\right)$-dimensional manifold transverse to $W^{s}(x)$ where $x \in \Lambda$.

5.1.1.C.I First application of the Lambda Lemma. First we apply the lambda lemma to the $\left(n_{s}\right)$-dimensional manifold $W^{s}\left(p^{+}\right)$passing through the point $p$. There exists a family of $\left(n_{s}\right)$-dimensional compact disks

$$
\mathscr{D}_{k^{-}}^{s}(p) \subset W^{s}\left(p^{+}\right)
$$

centered at $p$ such that $f^{-k^{-}}\left(\mathscr{D}_{k^{-}}^{S}(p)\right) C^{1}$-approaches as $k^{-} \rightarrow \infty$, a disk of fixed radius in $W^{s}\left(f^{-k^{-}}\left(p^{-}\right)\right)$and centered at $f^{-k^{-}}\left(p^{-}\right)$. Denote

$$
D^{s}\left(f^{-k^{-}}(p)\right):=f^{-k^{-}}\left(\mathscr{D}_{k^{-}}^{s}(p)\right) \subset W^{s}\left(f^{-k^{-}}\left(p^{+}\right)\right),
$$

the disk centered at $f^{-k^{-}}(p)$ that is asymptotic to a moving disk of fixed size in $W^{s}\left(f^{-k^{-}}\left(p^{-}\right)\right)$.

Choose $k^{-}$large enough and $\mathscr{D}_{k^{-}}^{s}(p)$ sufficiently small so that $D^{s}\left(f^{-k^{-}}(p)\right)$ is contained in $h\left(B_{\rho_{c}}\left(x_{c}^{-}\right) \times B_{\rho_{u}}(0) \times B_{\rho_{s}}(0)\right)$ and is $\delta / 2$-close to $W^{s}\left(f^{-k^{-}}\left(p^{-}\right)\right)$ in the $C^{1}$-topology.

Due to the compactness of $\Lambda$ and $\Gamma$, the size of the disk $D^{s}\left(f^{-k^{-}}(p)\right)$ can be chosen independently of $p \in \Gamma$ and of $k^{-}$. 
5.1.1.C.II First transversality property. Since $\mathscr{D}_{k^{-}}^{s}(p)$ is transverse to $W^{u}(\Lambda)$ at $p$ :

$$
D^{s}\left(f^{-k^{-}}(p)\right) \text { is transverse to } W^{u}(\Lambda) \text { at } f^{-k^{-}}(p) \text { for any } k^{-} \geq n^{*} .
$$

5.1.1.C.III Second application of the Lambda Lemma. We now apply the lambda lemma to the $\left(n_{c}+n_{u}\right)$-dimensional manifold $W^{u}(\Lambda)$ at the point $f^{-k^{-}}(p)$, which is transverse to $W^{s}\left(f^{-k^{-}}\left(p^{+}\right)\right)$at $f^{-k^{-}}(p)$. In particular, it is transverse to $D^{s}\left(f^{-k^{-}}(p)\right)$.

There exists a family of $\left(n_{c}+n_{u}\right)$-dimensional disks

$$
\mathscr{D}_{k^{-}, k^{+}}^{c u}\left(f^{-k^{-}}(p)\right) \subseteq W^{u}(\Lambda),
$$

centered at $f^{-k^{-}}(p)$, with each disk being a neighborhood of $f^{-k^{-}}(p)$ in $W^{u}(\Lambda)$, such that each $f^{k^{+}+k^{-}}\left(\mathscr{D}_{k^{-}, k^{+}}^{c u}\left(f^{-k^{-}}(p)\right)\right)$ approaches, in the $C^{1}$-topology as $k^{+} \rightarrow \infty$, a disk of fixed size in the unstable directions contained in $W^{u}(\Lambda)$ and centered at $f^{k+}\left(p^{+}\right)$as $k^{+} \rightarrow \infty$.

If we choose $k^{-}$and $k^{+}$large enough and fixed, for every disk

$$
D^{c u}\left(f^{-k^{-}}(p)\right):=h\left(B_{\rho_{c}^{-}}\left(x_{c}^{-}\right) \times B_{\rho_{u}^{-}}\left(v_{u}^{-}\right) \times\{0\}\right) \subseteq \mathscr{D}_{k^{-}, k^{+}}^{c u}\left(f^{-k^{-}}(p)\right)
$$

with $\rho_{c}^{-}>0$ and $\rho_{u}^{-}>0$ small enough, we have that $f^{k^{+}+k^{-}}\left(D^{c u}\left(f^{-k^{-}}(p)\right)\right)$ is $\delta / 2$-close, in the $C^{1}$-topology, to some disk of the form $h\left(B_{\rho_{c}^{+}}\left(x_{c}^{+}\right)\right) \times B_{\rho_{u}^{+}}(0) \times$ $\{0\})$ contained in $W^{u}(\Lambda)$ for some $\rho_{u}^{+}>0, \rho_{c}^{+}>0$. Define

$$
D^{c u}\left(f^{k^{+}}(p)\right):=f^{k^{+}+k^{-}}\left(D^{c u}\left(f^{-k^{-}}(p)\right)\right) .
$$

We have that $\rho_{u}^{+}>0$ depends on $\rho_{u}^{-}$and $\rho_{c}^{-}$but is independent of $k^{-}$and $k^{+}$ provided they are large enough, and $\rho_{c}^{+}>0$ depends on $\rho_{u}^{-}, \rho_{c}^{-}, k^{-}, k^{+}$. For $k^{-}, k^{+}$fixed, the smaller $\rho_{u}^{-}, \rho_{c}^{-}$, the smaller $\rho_{u}^{+}>0, \rho_{c}^{+}>0$.

Here we should note that, while $D^{c u}\left(f^{-k^{-}}(p)\right)$ is defined via the $h$-coordinates, which are only $C^{0}$, it is in fact contained in $W^{u}(\Lambda)$, so it is embedded in a $C^{1}$-disk. Hence we can measure its distance away from $W^{u}(\Lambda)$ in terms of the $C^{1}$-topology. Also, note that $D^{c u}\left(f^{k^{+}}(p)\right) \pitchfork W^{s}\left(f^{k^{+}}\left(p^{+}\right)\right)$. We derive the following:

5.1.1.C.IV Second transversality property. For $k^{+}$sufficiently large and fixed, there exist $\rho_{u}^{+}>0$ and $\rho_{c}^{+}>0$ such that for each $z_{c}^{+} \in B_{\rho_{c}^{+}}\left(x_{c}^{+}\right), w_{u}^{+} \in B_{\rho_{u}^{+}}(0)$,

$$
D^{c u}\left(f^{k^{+}}(p)\right) \text { is topologically transverse to } h\left(\left\{z_{c}^{+}\right\} \times\left\{w_{u}^{+}\right\} \times B_{\rho^{s}}(0)\right),
$$

where $\rho^{s}$ is defined in 5.1.

See [58] for a definition of topological transversality. Since the linearized coordinates $h$ are $C^{0}$, the $n_{s}$-disks $h\left(\left\{z_{c}^{+}\right\} \times\left\{w_{u}^{+}\right\} \times B_{\rho^{s}}(0)\right)$ in (5.7) are only $C^{0}$. This is why we have to use the notion of topological transversality rather than the differentiable one. Property (5.7) holds true for the following reasons. The $n_{s}$-disks $h\left(\left\{z_{c}^{+}\right\} \times\left\{w_{u}^{+}\right\} \times B_{\rho^{s}}(0)\right)$ depend in a $C^{0}$-fashion on $z_{c}^{+}$and $w_{u}^{+}$. For $z_{c}^{+}=x_{c}^{+}$ 
and $w_{u}^{+}=0$ the corresponding $n_{s}$-dimensional disk is a part of the stable fiber $W^{s}\left(f^{k^{+}}\left(p^{+}\right)\right)$, which is differentiably transverse to the $\left(n_{c}+n_{u}\right)$-dimensional disk $D^{c u}\left(f^{k^{+}}(p)\right)$. Differentiable transversality implies topological transversality, and topological transversality is $C^{0}$-stable.

Property (5.7) implies that

$$
\pi_{c, u}\left(h^{-1}\left(D^{c u}\left(f^{k^{+}}(p)\right)\right)\right) \supseteq B_{\rho_{c}^{+}}\left(x_{c}^{+}\right) \times B_{\rho_{u}^{+}}(0),
$$

where $\pi_{c, u}$ is the projection onto the $(c, u)$-subspace of $\left(E^{u} \oplus E^{s}\right) \Lambda$ relative to the $h$-coordinate system.

Due to the compactness of $\Lambda$ and $\Gamma, \rho_{u}^{+}$can be chosen independently of $p \in \Gamma$ and of $k^{-}, k^{+}$, provided they are large enough, but will depend on $\rho_{u}^{-}, \rho_{c}^{-}$, whereas $\rho_{c}^{+}$can be chosen independently of $p \in \Gamma$, but will depend on $\rho_{u}^{-}, \rho_{c}^{-}, k^{-}, k^{+}$.

5.1.1.D Choice of $n^{*}$. Fix $\delta>0$, and let $n^{*}>0$ be sufficiently large so that the conditions in Section $\mathrm{C}$ hold. We impose additional conditions on $n^{*}$.

Since $\Gamma$ is compact, we can choose $n^{*}>0$ such that for every $k^{-} \geq n^{*}$ and every $p \in \Gamma$, the $n_{s^{-}}$-dimensional compact disk $D^{s}\left(f^{-k^{-}}(p)\right)$ given in 5.3 always satisfies the transversality condition (5.4). In other words, $k^{-}$can be chosen uniformly with respect to $p \in \Gamma$. This $n^{*}$ is the number that appears in the statement of Lemma 3.1 .

Fix such an $n^{*}$ depending on $\delta$ and independent of $p \in \Gamma$.

For a fixed choice of $p \in \Gamma$ and of $k^{-}>n^{*}$, let $D^{c u}\left(f^{-k^{-}}(p)\right)$ be the disk attached to $f^{-k^{-}}(p)$ described in (5.5) for some $\rho_{c}^{-}>0, \rho_{u}^{-}>0$. For every $k^{+} \geq n^{*}$, the $\left(k^{-}+k^{+}\right)^{\text {th }}$ iterate of $D^{c u}\left(f^{-k^{-}}(p)\right)$, denoted by $D^{c u}\left(f^{k^{+}}(p)\right)$ in (5.6), satisfies (5.7) and (5.8) for some $\rho_{u}^{+}$and $\rho_{c}^{+}$. The power $k^{+}$can be chosen uniformly with respect to $p \in \Gamma$, and for $k^{+}$fixed, the parameters $\rho_{u}^{+}$and $\rho_{c}^{+}$depend on $\rho_{c}^{-}$and $\rho_{u}^{-}$.

It is also important to note that $k^{+}, \rho_{u}^{+}, \rho_{c}^{+}$also depend on the angle of the intersection between $W^{u}(\Lambda)$ and $W^{s}(\Lambda)$ at $p \in \Gamma$. When the angle of intersection is small, the radii $\rho_{u}^{+}$and $\rho_{c}^{+}$need to be chosen sufficiently small. However, our argument is only qualitative, and making quantitative estimates on the dependence of this product of disks on the angle of intersection is beyond the purpose of this paper. Since $\Gamma$ is compact, there exists a positive lower bound for the angle of intersection, and thus we can make the choices of $k^{-}$and $k^{+}$uniform for all points $p \in \Gamma$.

5.1.1.E Prototype windows. For $\delta>0$ fixed, choose and fix $n^{*}>0$ as in Section C. Consider a point $p$ in the homoclinic channel $\Gamma$. For fixed $k^{-}, k^{+} \geq n^{*}$ consider a pair of disks: the $n_{s}$-dimensional $D^{s}\left(f^{-k^{-}}(p)\right)$ as in $(5.3)$ and the $\left(n_{c}+n_{u}\right)$-dimensional $D^{c u}\left(f^{k^{+}}(p)\right)$ as in (5.6).

We make the following claim:

5.1.1.E.I Claim on $m^{*}$. There exists $m^{*} \geq 0$ depending on the size of the disks $D^{s}\left(f^{-k^{-}}(p)\right)$ and $D^{c u}\left(f^{k^{+}}(p)\right)$ such that for every $m \geq m^{*}$ and every $k^{\prime-} \geq$ 
$n^{*}$, if $p^{\prime} \in \Gamma$ is such that

$$
p^{\prime-}=f^{k^{\prime-}+m}\left(p^{+}\right)
$$

then there exists a triplet of windows $W^{-}, W^{+}, W^{\prime-}$ with the following properties:

- $W^{-}$is contained in a $\delta / 2$-neighborhood of $f^{-k^{-}}(p)$ and therefore in a $\delta$-neighborhood of $f^{-k^{-}}\left(p^{-}\right)$;

- $W^{+}$is contained in a $\delta$-neighborhood of $f^{k^{+}}\left(p^{+}\right)$;

- $W^{\prime-}$ is contained in a $\delta / 2$-neighborhood of $f^{-k^{\prime-}}\left(p^{\prime}\right)$ and therefore in a $\delta$-neighborhood of $f^{-k^{\prime-}}\left(p^{\prime-}\right)$;

- $W^{-}$is correctly aligned with $W^{+}$under $f^{k^{-}+k^{+}}$;

- $W^{+}$is correctly aligned with $W^{\prime-}$ under $f^{m-k^{+}}$;

- the sizes of the windows $W^{-}, W^{+}, W^{\prime-}$ do not depend on the points $p, p^{\prime} \in \Gamma$; the size of $W^{+}$depends only on the size of $W^{-}$and on $k^{-}, k^{+}$; the size of $W^{\prime-}$ depends only on the size of $W^{+}$and on $m$ and $k^{+}$.

In the above, $p^{-}, p^{+}$satisfy $W^{u}\left(p^{-}\right) \cap W^{s}\left(p^{+}\right) \cap \Gamma=\{p\}$, and $p^{\prime-}, p^{+}$ satisfy $W^{u}\left(p^{\prime-}\right) \cap W^{s}\left(p^{\prime+}\right) \cap \Gamma=\left\{p^{\prime}\right\}$. We will refer to $W^{-}, W^{+}, W^{\prime-}$ as prototype windows as we will use them in the next section to construct an infinite sequence of correctly aligned windows, as described in the outline.

5.1.1.E.II Construction of $W^{-}$. We construct the window $W^{-}$about $f^{-k^{-}}(p)=$ $h\left(x_{c}^{-}, v_{u}^{-}, 0\right)$, where $\left\|v_{u}^{-}\right\|<\delta_{1}$ (see (5.1p). Consider the $\left(n_{s}\right)$-dimensional disk $D^{s}\left(f^{-k^{-}}(p)\right)$ through $f^{-k^{-}}(p)$ given in (5.3), and the $\left(n_{c}+n_{u}\right)$-dimensional disk $D^{c u}\left(f^{k^{+}}(p)\right)$ through $f^{k^{+}}(p)$ given in (5.6).

We attach the $\left(n_{c}+n_{u}\right)$-dimensional disk

$$
D^{c u}\left(f^{-k^{-}}(p)\right)=f^{-k^{+}-k^{-}}\left(D^{c u}\left(f^{k^{+}}(p)\right)\right)
$$

of fixed size independent of $p$, to the point $f^{-k^{-}}(p)$; see (5.6).

Then choose a $C^{0}$-family of $n_{s}$-dimensional disks $\mathscr{D}^{s}(q)=\mathscr{D}^{s}\left(f^{-k^{-}}(p)\right)$ of fixed size independent of $p$, with $q \in D^{c u}\left(f^{-k^{-}}(p)\right)$ satisfying the following conditions:

- for $q=f^{-k^{-}}(p)$ the disk $\mathscr{D}^{s}(q)$ is contained in $D^{s}\left(f^{-k^{-}}(p)\right)$;

- for each $q \in D^{c u}\left(f^{-k^{-}}(p)\right)$, we have

$$
f^{k^{+}+k^{-}}\left(\mathscr{D}^{s}(q)\right) \subset h\left(\left\{z_{c}^{+}\right\} \times\left\{w_{u}^{+}\right\} \times B_{\rho^{s}}(0)\right),
$$

where $z_{c}^{+} \in B_{\rho_{c}^{+}}\left(x_{c}^{+}\right)$and $w_{u}^{+} \in B_{\rho_{u}^{+}}(0)$ are defined as in (5.7).

Observe that, by construction, for each $q \in D^{c u}\left(f^{-k^{-}}(p)\right), \mathscr{D}^{s}(q)$ is topologically transverse to $D^{c u}\left(f^{-k^{-}}(p)\right)$.

Summarizing, the $\left(n_{c}+n_{u}\right)$-dimensional disk $D^{c u}\left(f^{-k^{-}}(p)\right)$ is contained in $W^{u}(\Lambda)$, the $n_{s}$-dimensional disk $D^{s}\left(f^{-k^{-}}(p)\right)$ is $(\delta / 2)$-close in the $C^{1}$-topology to $W^{s}\left(f^{-k^{-}}\left(p^{-}\right)\right)$, and each disk $f^{k^{+}+k^{-}}\left(\mathscr{D}^{s}(q)\right)$ is topologically transverse to $D^{c u}\left(f^{k^{+}}(p)\right)$. 
We define the window $W^{-}$and its exit and entry sets $\left(W^{-}\right)^{\text {exit }}$ and $\left(W^{-}\right)^{\text {entry }}$, respectively, by

$$
\begin{aligned}
W^{-} & =\bigcup_{q \in D^{c u}\left(f^{-k^{-}}(p)\right)} \mathscr{D}^{s}(q), \\
\left(W^{-}\right)^{\text {exit }} & =\bigcup_{q \in \partial D^{c u}\left(f^{-k^{-}}(p)\right)} \mathscr{D}^{s}(q), \\
\left(W^{-}\right)^{\text {entry }} & =\bigcup_{q \in D^{c u}\left(f^{-k^{-}}(p)\right)} \partial \mathscr{D}^{s}(q) .
\end{aligned}
$$

We choose the sizes of $D^{c u}\left(f^{-k^{-}}(p)\right)$ and of $\mathscr{D}^{s}(q)$, for $q \in D^{c u}\left(f^{-k^{-}}(p)\right)$, such that $W^{-}$is contained in a $(\delta / 2)$-neighborhood of $f^{-k^{-}}(p)$; hence every point in $W^{-}$is $\delta$-close to $f^{-k^{-}}\left(p^{-}\right)$.

We note that $W^{-}$is a window; see Remark C.2.

We will impose additional conditions on the sizes of $D^{c u}\left(f^{-k^{-}}(p)\right)$ and $\mathscr{D}^{s}(q)$ in Section 5.1.1.E.III.

We take a forward iterate $f^{k^{+}+k^{-}}\left(W^{-}\right)$of $W^{-}$. The point $f^{-k^{-}}(p)$ is mapped by $f^{k^{+}+k^{-}}$onto $f^{k^{+}}(p)$. For $k^{+} \geq n^{*}$ we have $f^{k^{+}}(p) \in V_{\Lambda}$. The set $f^{k^{+}+k^{-}}\left(W^{-}\right)$is still a window, since it is a homeomorphic copy of $W^{-}$under $f^{k^{+}+k^{-}}$, with the exit and entry sets being defined by transporting the exit and entry sets of $W^{-}$, respectively, through $f^{k^{+}+k^{-}}$.

In fact, by construction

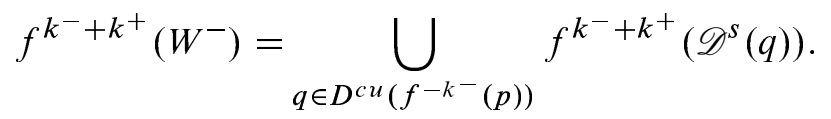

5.1.1.E.III Construction of $W^{+}$. We construct a new window $W^{+} \subseteq V_{\Lambda}$ about $f^{k^{+}}\left(p^{+}\right)=h\left(x_{c}^{+}, 0,0\right)$ such that $f^{k^{+}+k^{-}}\left(W^{-}\right)$is correctly aligned with $W^{+}$ under the identity map, or, equivalently, $W^{-}$is correctly aligned with $W^{+}$under $f^{k^{+}+k^{-}}$. This new window will be a product of disks in the linearized coordinates $h$. The construction follows below.

The image set $D^{c u}\left(f^{k^{+}}(p)\right):=f^{k^{+}+k^{-}}\left(D^{c u}\left(f^{-k^{-}}(p)\right)\right)$ is a $\left(n_{c}+n_{u}\right)$ dimensional disk through $f^{k^{+}}(p)$ that is $(\delta / 2)$-close to $W^{u}(\Lambda)$; see (5.6). This disk is transverse to $W^{s}\left(f^{k^{+}}\left(p^{+}\right)\right)$. Also denote

$$
D^{s}\left(f^{k^{+}}(q)\right):=f^{k^{+}+k^{-}}\left(\mathscr{D}^{s}(q)\right), \quad q \in D^{c u}\left(f^{-k^{-}}(p)\right) .
$$

For a given choice of the size of $D^{c u}\left(f^{-k^{-}}(p)\right)$, we require $\rho_{c}^{+}>0$ and $\rho_{u}^{+}>0$ to be sufficiently small, so that (5.7) and (5.8) hold. We also require that $\rho_{c}^{+}, \rho_{u}^{+}<\delta_{1}$.

Then we choose $0<\rho_{s}^{+}<\delta_{1}$ and require that all disks $\mathscr{D}^{s}(q)$ be small enough so that

$$
\pi_{s}\left[h^{-1}\left(D^{s}\left(f^{k^{+}}(q)\right)\right)\right] \subseteq \operatorname{int}\left[B_{\rho_{s}^{+}}(0)\right]
$$


for all $q \in D^{c u}\left(f^{-k^{-}}(p)\right)$.

For future reference, we also have to set a lower bound for the sizes of the disks $\mathscr{D}^{s}(q), q \in D^{c u}\left(f^{-k^{-}}(p)\right)$. There exist $\delta_{2}>0$ defined by the property that

$$
\operatorname{int}\left[\pi_{s}\left(h^{-1}\left(\mathscr{D}^{s}(q)\right)\right)\right] \supseteq B_{\delta_{2}}(0)
$$

for all $q \in D^{c u}\left(f^{-k^{-}}(p)\right)$.

We now define the second "prototype" window $W^{+}$around $f^{k^{+}}\left(p^{+}\right)$to be given in the $h$-coordinates by

$$
\begin{gathered}
W^{+}=h\left[B_{\rho_{c}^{+}}\left(x_{c}^{+}\right) \times B_{\rho_{u}^{+}}(0) \times B_{\rho_{s}^{+}}(0)\right], \\
\left(W^{+}\right)^{\text {exit }}=h\left[\partial B_{\rho_{c}^{+}}\left(x_{c}^{+}\right) \times B_{\rho_{u}^{+}}(0) \times B_{\rho_{s}^{+}}(0)\right. \\
\left.\cup B_{\rho_{c}^{+}}\left(x_{c}^{+}\right) \times \partial B_{\rho_{u}^{+}}(0) \times B_{\rho_{s}^{+}}(0)\right], \\
\left(W^{+}\right)^{\text {entry }}=h\left[B_{\rho_{c}^{+}}\left(x_{c}^{+}\right) \times B_{\rho_{u}^{+}}(0) \times \partial B_{\rho_{s}^{+}}(0)\right] .
\end{gathered}
$$

By the product property of correct alignment, Lemma C.5, the choices that we made imply that $W^{-}$is correctly aligned with $W^{+}$under $f^{k^{+}}+k^{-}$.

It is useful at this point to summarize the interdependence of the parameters involved in the construction of the windows $W^{-}$and $W^{+}$so that they are correctly aligned under $f^{k^{-}+k^{+}}$.

- The quantities $\rho_{c}^{+}, \rho_{u}^{+}, \rho_{s}^{+}$from above can be chosen independently of the point $p \in \Gamma$, but they depend on $k^{-}, k^{+}$on the sizes of the disks involved in the definition of the window $W^{-}$.

- The powers $k^{-}, k^{+}$can be chosen arbitrarily large with $k^{-} \geq n^{*}$ and $k^{+} \geq n^{*}$, where $n^{*}$ depends only on $\delta$ and not on $p \in \Gamma$.

- The disks $D^{c u}\left(f^{-k^{-}}(p)\right)$ and $D^{c u}\left(f^{k^{+}}(p)\right)$, and implicitly the parameters $\rho_{c}^{+}$and $\rho_{u}^{+}$, depend on $k^{-}$and $k^{+}$. In particular, for fixed $k^{-}$and $k^{+}$, the parameters $\rho_{c}^{+}$and $\rho_{u}^{+}$depend on the size of the disk $D^{c u}\left(f^{-k^{-}}(p)\right)$; the smaller the disk $D^{c u}\left(f^{-k^{-}}(p)\right)$ is, the smaller $\rho_{c}^{+}$and $\rho_{u}^{+}$need to be chosen. This is due to the coupling of the center and hyperbolic dynamics, which mixes the center and unstable directions when iterated along the homoclinic orbit. That is, the center and unstable directions of a disk are not preserved when the disk is iterated along a homoclinic orbit, as they "get mixed," therefore, the image of a center-unstable rectangle iterated along the stable manifold of a point does not remain a rectangle anymore, as the rectangle "gets distorted."

- The disks $\mathscr{D}^{s}(q), q \in D^{c u}\left(f^{-k^{-}}(p)\right)$, and implicitly the parameter $\delta_{2}$ in (5.12) depend on $k^{-}$and $k^{+}$. The sizes of these disks can be chosen independently of the size of the disk $D^{c u}\left(f^{-k^{-}}(p)\right)$ provided this is sufficiently small. That is, if $D^{c u}\left(f^{-k^{-}}(p)\right)$ is replaced by a smaller disk $\tilde{D}^{c u}\left(f^{-k^{-}}(p)\right) \subset D^{c u}\left(f^{-k^{-}}(p)\right)$, then we simply restrict the family of 
disks $\mathscr{D}^{s}(q)$ to those $q \in \widetilde{D}^{c u}\left(f^{-k^{-}}(p)\right)$ without having to modify the size of the disks $\mathscr{D}^{S}(q)$.

- Parameter $\rho_{s}^{+}$can be chosen independently of $k^{-}$and $k^{+}$provided that the disks $\mathscr{D}^{s}\left(f^{-k^{-}}(q)\right), q \in D^{c u}\left(f^{-k^{-}}(p)\right)$, are chosen small enough,

5.1.1.E.IV Choice of $m^{*}$. Now we show that there exists a number $m^{*}$ with the property that for every $m \geq m^{*}$ and every $k^{\prime-} \geq n^{*}$, and for every point $p^{\prime} \in \Gamma$ with $p^{\prime-}=f^{m+k^{\prime-}}\left(p^{+}\right)$, we can construct a window $W^{\prime-}$ near $f^{-k^{\prime-}}\left(p^{\prime}\right)$ in a similar way to which we have constructed $W^{-}$such that $W^{+}$is correctly aligned with $W^{\prime-}$ under $f^{m-k^{+}}$.

Since the power $m-k^{+}$should be nonnegative, we first require $m^{*} \geq k^{+}$. We also fix $k^{\prime+}=k^{+} \geq n^{*}$.

A key observation is that, since $W^{+}$is a window of product type relative to the $h$-coordinates, the image $f^{m^{\prime}}\left(W^{+}\right)$is also a window of product type relative to the $h$-coordinates, for any iterate $f^{m^{\prime}}$, provided that $f^{k}\left(W^{+}\right)$remains in the domain $V_{\Lambda}$ of the map $h$ for $0 \leq k \leq m^{\prime}$. This is due to the fact that, relative to the linearized coordinates, the map $f$ is conjugate to $N f$ (see Appendix B).

Even in the case when $f^{m^{\prime}}\left(W^{+}\right)$does not entirely remain in $V_{\Lambda}$ (e.g., it "escapes" in the unstable directions), $f^{m^{\prime}}\left(W^{+}\right) \cap V_{\Lambda}$ contains a subwindow of product type, say $\widetilde{W}$. If this window $\widetilde{W}$ is correctly aligned with $W^{\prime-}$ under the identity map, it immediately follows that $f^{m^{\prime}}\left(W^{+}\right)$itself is correctly aligned with $W^{\prime-}$. So for all practical purposes we can assume that $f^{m^{\prime}}\left(W^{+}\right)$stays in $V_{\Lambda}$.

We now take $\delta_{1}$ from (5.1) and $\delta_{2}$ from (5.12). By (5.2), there exists $m^{*} \geq k^{+}$ large enough so that for $m^{\prime} \geq m^{*}-k^{+}, f^{m^{\prime}}\left(h\left(\left\{x_{c}^{+}\right\} \times B_{\rho_{u}^{+}}(0) \times\{0\}\right)\right)$ contains a disk in $W^{u}\left(h\left(\left\{f^{m^{\prime}}\left(x_{c}^{+}\right)\right\} \times\{0\} \times\{0\}\right)\right)$ of radius $\delta_{1}$ relative to the $h$-coordinates, that is:

$$
\operatorname{int}\left[f^{m^{\prime}}\left(h\left(\left\{x_{c}^{+}\right\} \times B_{\rho_{u}^{+}}(0) \times\{0\}\right)\right)\right] \supseteq h\left(\left\{f^{m^{\prime}}\left(x_{c}^{+}\right)\right\} \times B_{\delta_{1}}(0) \times\{0\}\right),
$$

and $f^{m^{\prime}}\left(h\left(\left\{x_{c}^{+}\right\} \times\{0\} \times B_{\rho_{s}^{+}}(0)\right)\right)$ is contained in a disk in $W^{s}\left(h\left(\left\{f^{m^{\prime}}\left(x_{c}^{+}\right)\right\} \times\right.\right.$ $\{0\} \times\{0\}))$ of radius $\delta_{2}$, that is,

$$
f^{m^{\prime}}\left[h\left(\left\{x_{c}^{+}\right\} \times\{0\} \times B_{\rho_{s}^{+}}(0)\right)\right] \subseteq \operatorname{int}\left[h\left(\left\{f^{m^{\prime}}\left(x_{c}^{+}\right)\right\} \times\{0\} \times B_{\delta_{2}}(0)\right)\right] .
$$

Observe that the parameter $\delta_{2}$ in (5.12) depends on $k^{\prime-}$ and $k^{\prime+}=k^{+}$.

Fix $m^{*}$ with these properties. Note that $m^{*}$ depends, in particular, on the size of the unstable component $B_{\rho_{u}^{+}}(0)$ of the previous window $W^{+}$, which in turn depends on the size of the disk $D^{c u}\left(f^{-k^{-}}(p)\right)$ that is used in the construction of the first window $W^{-}$; the smaller the radius $\rho_{u}^{+}$is, the larger $m^{*}$ needs to be chosen in order to satisfy 5.13. 
5.1.1.E.V Construction of $W^{\prime-}$. Let $m \geq m^{*}$ and let $m^{\prime}=m-k^{+}$. Assume that $p^{\prime} \in \Gamma$ is such that $p^{\prime-}=f^{m+k^{\prime-}}\left(p^{+}\right)$. We construct a third window $W^{\prime-}$ near $f^{-k^{\prime-}}\left(p^{\prime}\right)$ in a similar way to the construction of $W^{-}$such that $W^{+}$is correctly aligned under $f^{m^{\prime}}$ with $W^{\prime-}$.

Consider the point $f^{-k^{\prime-}}\left(p^{\prime}\right) \in W^{u}\left(f^{-k^{\prime-}}\left(p^{\prime-}\right)\right)$. Choose a sufficiently small $\left(n_{c}+n_{u}\right)$-dimensional disk $\tilde{D}^{c u}\left(f^{-k^{\prime-}}\left(p^{\prime}\right)\right) \subseteq \mathscr{D}_{k^{\prime}, k^{\prime+}}^{c u}\left(f^{-k^{\prime-}}\left(p^{\prime}\right)\right)$ in $W^{u}(\Lambda)$ such that it contains the point $f^{-k^{\prime}}\left(p^{\prime}\right)$ and satisfies the following condition:

$$
\begin{aligned}
\pi_{c, u}\left[h^{-1}\left(\widetilde{D}^{c u}\left(f^{-k^{\prime-}}\left(p^{\prime}\right)\right)\right)\right] \subseteq & \\
& \operatorname{int}\left[h^{-1} \circ f^{m^{\prime}} \circ h\left(B_{\rho_{c}^{+}}\left(x_{c}^{+}\right) \times B_{\rho_{u}^{+}}(0) \times\{0\}\right)\right] .
\end{aligned}
$$

The size of the disk $\widetilde{D}^{c u}\left(f^{-k^{\prime-}}\left(p^{\prime}\right)\right)$ can be chosen to depend only on the window $W^{+}$, on $m^{\prime}$ and $\delta_{1}$ in (5.1), and independently of the point $p^{\prime} \in \Gamma$. Then we choose a $C^{0}$-family of $n_{s}$-dimensional disks $\widetilde{\mathscr{D}}^{s}\left(q^{\prime}\right)$, with $q^{\prime} \in \widetilde{D}^{c u}\left(f^{-k^{\prime-}}\left(p^{\prime}\right)\right)$, such that for $\delta_{2}$ in (5.12)

$$
\operatorname{int}\left[\pi_{s}\left(h^{-1}\left(\widetilde{\mathscr{D}}^{s}\left(q^{\prime}\right)\right)\right)\right] \supseteq B_{\delta_{2}}(0)
$$

for all $q^{\prime} \in \widetilde{D}^{c u}\left(f^{-k^{\prime-}}\left(p^{\prime}\right)\right)$, and when $q^{\prime}=f^{-k^{\prime-}}\left(p^{\prime}\right)$,

$$
\widetilde{\mathscr{D}}^{s}\left(f^{-k^{\prime-}}\left(p^{\prime}\right)\right) \subset W^{s}\left(f^{-k^{\prime-}}\left(p^{\prime+}\right)\right) .
$$

As noted earlier, $\delta_{2}$ is independent of the choice of the disk $\widetilde{D}^{c u}\left(f^{-k^{\prime-}}\left(p^{\prime}\right)\right)$ provided this is sufficiently small, and only depends on $k^{\prime-}$ and $k^{\prime+}=k^{+}$. For fixed $k^{\prime-}, k^{\prime+}$, and $\delta_{2}$ sufficiently small, a family of disks $\widetilde{\mathscr{D}^{s}}\left(q^{\prime}\right)$ satisfying (5.16) can always be constructed.

Conditions (5.14) and (5.16) imply that the projection of each $\widetilde{D^{s}}\left(q^{\prime}\right)$ for $q^{\prime} \in$ $\widetilde{D}^{c u}\left(f^{-k^{\prime-}}\left(p^{\prime}\right)\right)$ onto the stable coordinates contains the stable component of $f^{m^{\prime}}\left(W^{+}\right)$inside it, that is,

$$
\operatorname{int}\left[\pi_{s}\left(h^{-1}\left(\widetilde{D}^{s}\left(q^{\prime}\right)\right)\right)\right] \supseteq h^{-1} \circ f^{m^{\prime}} \circ h\left(\left\{z_{c}^{+}\right\} \times\{0\} \times B_{\rho_{s}^{+}}(0)\right),
$$

for all $z_{c}^{+} \in B_{\rho_{c}^{+}}\left(x_{c}^{+}\right)$.

The window $W^{\prime-}$ is then defined similarly to $W^{-}$, by

$$
\begin{aligned}
W^{\prime-} & =\underset{q^{\prime} \in \tilde{D}^{c u}\left(f^{-k^{\prime}-}\left(p^{\prime}\right)\right)}{W^{\prime \text { exit }}}=\widetilde{\mathscr{D}}^{s}\left(q^{\prime}\right), \\
W^{\prime \text { entry }}=\partial \widetilde{D}^{c u}\left(f^{-k^{\prime}-}\left(p^{\prime}\right)\right) & \bigcup_{q^{s}}\left(q^{\prime}\right), \\
q^{\prime} \in \tilde{D}^{c u}\left(f^{-k^{\prime}}\left(p^{\prime}\right)\right) & \partial \widetilde{D}^{s}\left(q^{\prime}\right) .
\end{aligned}
$$

Conditions (5.15) and (5.17) imply that the product property of correct alignment applies (Lemma C.5), and hence we obtain that $W^{+}$is correctly aligned 


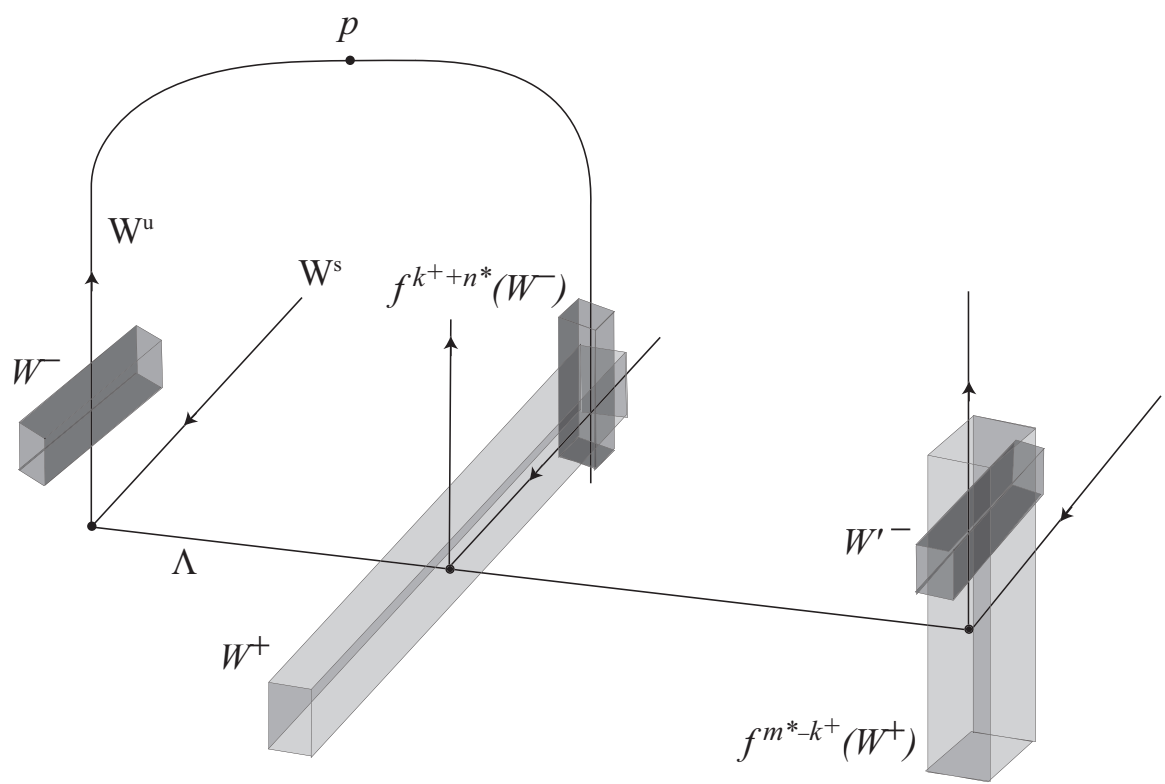

FIGURE 5.1. Construction of windows.

under $f^{m^{\prime}}$ with $W^{\prime-}$. An important point to keep in mind is that we have no control on the size of the $\left(n_{c}+n_{u}\right)$-dimensional disk $\widetilde{D}^{c u}\left(f^{-k^{\prime-}}\left(p^{\prime}\right)\right)$ involved in the construction $W^{\prime-}$. We choose this disk so that its center-unstable part is contained in the center-unstable component of $f^{m^{\prime}}\left(W^{+}\right)$. Thus, the size of the disk $\tilde{D}^{c u}\left(f^{-k^{\prime-}}\left(p^{\prime}\right)\right)$ utilized in the construction $W^{\prime-}$ may be smaller than the size of the disk $D^{c u}\left(f^{-k^{\prime-}}(p)\right)$ utilized in the construction of $W^{-}$.

A schematic representation of the construction of the triplet of windows $W^{-}$, $W^{+}, W^{\prime-}$ constructed so far is shown in Figure 5.1 .

We anticipate that, in order to continue this construction of triplets of correctly aligned windows starting from $W^{\prime-}$, the number of iterates $m^{\prime *}$ that we need to choose at the next step, in order to satisfy [5.13), may need to be larger than $m^{*}$. Without further conditions on the dynamics, we cannot guarantee a uniform choice of $m^{*}$ to work for all steps of the construction. In Appendix D we show an example, which was kindly communicated to us by Dmitry Turaev, showing that a "uniform" version of this shadowing lemma is not true in general.

5.1.1.F Definition of $m_{i}^{*}$. Let $p_{0} \in \Gamma$ be an arbitrary homoclinic point, and let $n_{0} \geq n_{*}$. We construct a pair of windows $W_{0}^{-}$in a $\delta / 2$-neighborhood of $f^{-n_{0}}\left(p_{0}\right)$, and $W_{0}^{+}$in a $\delta$-neighborhood of $f^{k^{+}}\left(p_{0}^{+}\right)$, where $k^{+} \geq n^{*}$ is fixed, such that $W_{0}^{-}$is correctly aligned with $W_{0}^{+}$under $f^{n_{0}+k^{+}}$. Then, the procedure in Section 5.1.1.E.IV provides an $m_{0}^{*}=m_{0}^{*}\left(n_{0}\right)$ that depends on $W_{0}^{-}$and $W_{0}^{+}$, and implicitly on $n_{0}$, and is independent of $p_{0} \in \Gamma$. Inductively, if $m_{0}^{*}, \ldots, m_{i-1}^{*}$ 
have been defined, let

$$
\begin{aligned}
n_{0} & \geq n^{*}, \ldots, n_{i-1} \geq n^{*}, n_{i} \geq n^{*}, \\
m_{0} & \geq m_{0}^{*}\left(n_{0}\right) \\
& \vdots \\
m_{i-1} & \geq m_{i-1}^{*}\left(n_{0}, \ldots, n_{i-1}, m_{0}, \ldots, m_{i-2}\right) .
\end{aligned}
$$

Let

$$
p_{0}, \ldots, p_{i} \in \Gamma
$$

such that $f^{n_{j}+m_{j-1}}\left(p_{j-1}^{+}\right)=p_{j}^{-}$for $j=1, \ldots, i$.

Let

$$
W_{0}^{-}, W_{0}^{+}, W_{1}^{-}, \ldots, W_{i}^{-}, W_{i}^{+},
$$

be a sequence of correctly aligned windows, constructed as above, such that

- $W_{j-1}^{-}$is in a $\delta / 2$-neighborhood of $f^{-n_{j-1}}\left(p_{j-1}\right)$,

- $W_{j-1}^{+}$is in a $\delta$-neighborhood of $f^{k^{+}}\left(p_{j-1}^{+}\right)$,

- $W_{j-1}^{-}$is correctly aligned with $W_{j-1}^{+}$under $f^{n_{j-1}+k^{+}}$, and

- $W_{j-1}^{+}$is correctly aligned with $W_{j}^{-}$under $f^{m_{j-1}-k^{+}}$, for $j=1, \ldots, i$.

Then the procedure in Section 5.1.1.E.IV provides an

$$
m_{i}^{*}=m_{i}^{*}\left(n_{0}, \ldots, n_{i-1}, n_{i}, m_{0}, \ldots, m_{i-1}\right)
$$

as in the statement of Lemma 3.1 , that depends on $W_{0}^{-}, \ldots, W_{i}^{+}$; hence on $n_{0}, \ldots$, $n_{i-1}, n_{i}, m_{0}, \ldots, m_{i-1}$, but is independent of $p_{0}, \ldots, p_{i-1} \in \Gamma$.

5.1.1.G Construction of an infinite sequence of correctly aligned windows.

Take a pseudo-orbit $\left\{y_{i}\right\}_{i \geq 0}$ as in the statement of Lemma 3.1. We implicitly assume that $f^{n_{i}}\left(y_{i}\right)$ is in the domain $\Omega^{-}(\Gamma)$ of $\sigma$, and hence $\sigma \circ f^{n_{i}}\left(y_{i}\right)$ is in the range $\Omega^{+}(\Gamma)$ of $\sigma$. Thus $W^{u}\left(f^{n_{i}}\left(y_{i}\right)\right) \cap W^{s}\left(\sigma\left(f^{n_{i}}\left(y_{i}\right)\right) \cap \Gamma=\left\{p_{i}\right\}\right.$ for some uniquely defined homoclinic point $p_{i} \in \Gamma$. Fix $k^{+} \geq n^{*}$.

Starting with the homoclinic point $p_{0}$ we construct inductively an infinite sequence of correctly aligned windows along the pseudo-orbit,

$$
W_{0}^{-}, W_{0}^{+}, W_{1}^{-}, \ldots, W_{i}^{-}, W_{i}^{+}, W_{i+1}^{-}, W_{i+1}^{+}, \ldots,
$$

such that for each $i \geq 0$ we have (choosing $p_{i}^{-}=f^{n_{i}}\left(y_{i}\right), p_{i}^{+}=\sigma\left(f^{n_{i}}\left(y_{i}\right)\right)$, $k_{i}^{-}=n_{i}$, and $k^{+}$fixed)

- $W_{i}^{-}$lies within a $\delta$-neighborhood of $y_{i}$;

- $W_{i}^{+}$lies within a $\delta$-neighborhood of $f^{k^{+}} \circ \sigma \circ f^{n_{i}}\left(y_{i}\right)$, where $n_{i} \geq n^{*}$;

- $W_{i}^{-}$is correctly aligned with $W_{i}^{+}$under $f^{k^{+}+n_{i}}$;

- $W_{i+1}^{-}$lies within a $\delta$-neighborhood of $y_{i+1}=f^{m_{i}} \circ \sigma \circ f^{n_{i}}\left(y_{i}\right)$;

- $W_{i}^{+}$is correctly aligned with $W_{i+1}^{-}$under $f^{m_{i}-k^{+}}$, where $m_{i} \geq m_{i}^{*}$. 
The shadowing property of correctly aligned windows, Theorem C.4, implies that there exists a point $z_{0} \in W_{0}^{-}$whose forward orbit visits all windows in the prescribed order. In particular, the orbit points given by $z_{i+1}=f^{m_{i}+n_{i}}\left(z_{i}\right)$ satisfy $z_{i} \in W_{i}^{-}$for all $i \geq 0$. Since each $W_{i}^{-}$is contained inside a $\delta$-neighborhood of $y_{i}$, it follows that $d\left(z_{i}, y_{i}\right)<\delta$ for all $i \geq 0$.

\subsubsection{A Proof Using the Obstruction Property}

In this section we give an alternative proof of Theorem 3.1 .

5.1.2.A Outline. The proof is based on the construction of a nested sequence of closed balls $B_{i+1} \subset B_{i}$ in a neighborhood of the first point of the pseudo-orbit $y_{0}$ such that taking $z_{0} \in B_{k}=\bigcap_{0 \leq i \leq k} B_{i}$ one has that $z_{0} \in B_{\delta}\left(y_{0}\right)$ and $z_{i+1}=$ $f^{m_{i}+n_{i}}\left(z_{0}\right) \in B_{\delta}\left(y_{i+1}\right)$ for $i=0,1 \ldots, k$, for any $k \in \mathbb{N}$. Moreover, taking $z_{0} \in B_{\infty}=\bigcap_{i \geq 0} B_{i} \neq \varnothing$, one has that $z_{i+1} \in B_{\delta}\left(y_{i+1}\right)$ for any $i \in \mathbb{N}$.

The argument will be done by induction.

We will define the values of $n^{*}$ and $m^{*}$ at every step of the induction process. We will see that $n^{*}$ can be taken once and for all but $m^{*}$ will depend on the previous choices.

5.1.2.B Choice of $n^{*}$ and $m^{*}$. Consider the homoclinic channel $\Gamma$ and the corresponding scattering map $\sigma: \Omega^{-}(\Gamma) \rightarrow \Omega^{+}(\Gamma)$. We will choose $\delta>0$ and consider $V_{\Lambda}$ and $V_{\Gamma}$ contained in neighborhoods of size $\delta$ of the compact manifolds $\Lambda$ and $\Gamma$, respectively.

We define $n^{*}=n^{*}(\delta)$ to be the same number as in Section 5.1.1.C In particular, given any point $p \in \Gamma$, for any $n \in \mathbb{N}$ with $n \geq n^{*}$, one has that $f^{ \pm n}(p) \in V_{\Lambda}$. Moreover, this property also holds for points in $W^{u, s}(\Lambda) \cap V_{\Gamma}$ when iterating them backwards or forward, respectively.

Moreover, we will modify $n^{*}$ to have the following additional property. Assume we have $p \in \Gamma$ and let $p^{-}, p^{+} \in \Lambda$ be the unique points for which $W^{u}\left(p^{-}\right) \cap$ $W^{s}\left(p^{+}\right) \cap \Gamma=\{p\}$.

(1) Let a point $x \in W^{s}\left(f^{-k^{-}}\left(p^{-}\right)\right)$and $B \subset B_{\delta}\left(f^{-k^{-}}\left(p^{-}\right)\right)$be any ball centered at $x$ of fixed radius $\rho>0$ small enough. Then we have that

$$
B \subset V_{\Lambda}, \quad x \in B \cap W^{s}\left(f^{-k^{-}}\left(p^{-}\right)\right) \neq \varnothing .
$$

As $W^{s}\left(p^{+}\right)$intersects transversally $W^{u}(\Lambda)$ at the homoclinic point $p$, by the lambda lemma, there exists a point $\bar{x} \in W^{s}\left(p^{+}\right) \cap V_{\Gamma}$ such that $f^{-k^{-}}(\bar{x}) \in B$ if $k^{-}>n^{*}$. The value of $n_{*}$ depends on $\rho$, which is fixed once and for all, and also on the angle of intersection of the stable and unstable manifolds of $\Lambda$ along $\Gamma$ which, by the hypothesis of compactness, is bounded below by a fixed quantity.

(2) By continuity, a ball $V \subset V_{\Gamma}$ exists centered at $\bar{x}$ such that $f^{-k^{-}}(\bar{x}) \in$ $f^{-k^{-}}(V) \subset B$.

The value of $n^{*}$ will be fixed from now on. Now we explain how we choose $m^{*}$ at every step of the process. 
Assume that we also have $p^{\prime} \in \Gamma$ and $p^{\prime-}, p^{\prime+}$ with the same properties as $p$ and $p^{-}, p^{+}$, and such that $f^{m+k^{\prime-}}\left(p^{+}\right)=p^{\prime-}$. Equivalently,

$$
p^{+}=f^{-\left(k^{-}+m\right)}\left(p^{-}\right) \text {. }
$$

Take the point $\bar{x} \in W^{s}\left(p^{+}\right)$and the ball $\bar{x} \in V \subset V_{\Gamma}$ centered at $\bar{x}$. Then choose $k^{+} \geq n^{*}$. The value of $k^{+}$will be fixed along the process.

(1) We know that $f^{k^{+}}(\bar{x}) \in B_{\delta}\left(f^{k^{+}}\left(p^{+}\right)\right) \cap V_{\Lambda} \cap W^{s}\left(f^{k^{+}}\left(p^{+}\right)\right)$, and there exists a ball $U$ centered at $f^{k^{+}}(\bar{x})$ such that

$$
\begin{aligned}
U \subset B_{\delta}\left(f^{k^{+}}\left(p^{+}\right)\right) & \subset V_{\Lambda}, \\
f^{k^{+}}(\bar{x}) \in U \cap W^{s}\left(f^{k^{+}}\left(p^{+}\right)\right) & \neq \varnothing, \\
f^{-k^{+}}(U) & \subset V .
\end{aligned}
$$

(2) As, by 5.20), $\left.f^{k^{+}}\left(p^{+}\right)=f^{-\left(k^{-}+m-k^{+}\right)}\left(p^{-}\right)\right)$, the ball $U$ satisfies

$$
\left.f^{k^{+}}(\bar{x}) \in U \cap W^{s}\left(f^{-\left(k^{\prime-}+m-k^{+}\right)}\left(p^{\prime-}\right)\right)\right) \neq \varnothing .
$$

(3) Now we apply the lambda lemma to $U$; we know that $W^{s}\left(p^{\prime+}\right)$ intersects $W^{u}(\Lambda)$ transversally at $p^{\prime}$, and therefore, if $k^{\prime-}+m-k^{+}>m^{*}$ is large enough (depending on the size of $U$ ), there exists $\bar{x}^{\prime} \in W^{s}\left(p^{\prime+}\right)$ such that $f^{-\left(k^{\prime-}+m-k^{+}\right)}\left(\bar{x}^{\prime}\right) \in U$.

(4) By continuity, there exists a ball centered at $\bar{x}^{\prime} \in V^{\prime} \subset V_{\Gamma}$ such that $f^{-\left(k^{\prime-}+m-k^{+}\right)}\left(V^{\prime}\right) \subset U$.

In summary, given a point $x \in W^{s}\left(f^{-k^{-}}\left(p^{-}\right)\right)$and a ball $B$ centered at $x$ of fixed radius $\rho>0$ small enough with the property that

$$
\begin{gathered}
B \subset B_{\delta}\left(f^{k^{-}}\left(p^{-}\right)\right) \subset V_{\Lambda}, \\
x \in B \cap W^{s}\left(f^{-k^{-}}\left(p^{-}\right)\right) \neq \varnothing,
\end{gathered}
$$

we have produced the following:

(1) for $k^{-} \geq n^{*}$, a ball $V \subset V_{\Gamma}$, centered at a point $\bar{x} \in W^{s}\left(p^{+}\right) \cap V_{\Gamma}$ such that $f^{-\bar{k}^{-}}(V) \subset B$;

(2) for $k^{+} \geq n^{*}$ and fixed, a ball $U \subset B_{\delta}\left(f^{k^{+}}\left(p^{+}\right)\right) \subset V_{\Lambda}$ centered at the point $f^{k^{+}}(\bar{x}) \in W^{s}\left(f^{k^{+}}\left(p^{+}\right)\right) \cap U$ such that $f^{-k^{+}}(U) \subset V$;

(3) for $k^{\prime-}+m-k^{+} \geq m^{*}$, a ball $V^{\prime} \subset V_{\Gamma}$, centered at a point $\bar{x}^{\prime} \in$ $W^{s}\left(p^{\prime+}\right) \cap V_{\Gamma}$ such that $f^{-\left(k^{\prime-}+m-k^{+}\right)}\left(V^{\prime}\right) \subset U$;

(4) moreover, as $k^{\prime-} \geq n^{*}$, we can also ensure $f^{-k^{\prime-}}\left(V^{\prime}\right) \subset B_{\delta}\left(f^{k^{\prime-}}\left(p^{\prime-}\right)\right)$. The values of $k^{+}, k^{-}, k^{\prime-}$ are taken bigger than $n^{*}$, which is already fixed, but the value of $m^{*}$ depends on the size of $U$ and $m^{*}>n^{*}$, but it is independent of the points $p, p^{\prime}, p^{ \pm},\left(p^{\prime}\right)^{ \pm}$. As the balls $U$ and $V$ will decrease in size during the induction process, the value of $m^{*}$ will increase depending on the previous iterates. 
5.1.2. Inductive construction. Now we begin the construction of the shadowing orbit $\left\{z_{i}\right\}$ once the pseudo-orbit $\left\{y_{i}\right\}$ is given. The required values of $n^{*}$ and $k^{+}$ are fixed (one can use, for instance, $k^{+}=n^{*}$ ), and $m_{i}^{*}$ does not depend on the given pseudo-orbit, but only on the numbers $n_{i}$ and $m_{j}$.

The first step in the induction procedure is done separately because it requires a slightly different reasoning. In this first step, $p^{-}=f^{n_{0}}\left(y_{0}\right), p^{+}=\sigma\left(f^{n_{0}}\left(y_{0}\right)\right)$, and $k^{-}=n_{0}$.

(1) Choose $x_{0} \in W^{s}\left(y_{0}\right)$, and let $B_{0}$ be any ball centered at $x_{0}$ of fixed radius $\rho>0$ such that

$$
\begin{gathered}
B_{0} \subset B_{\delta}\left(y_{0}\right) \subset V_{\Lambda}, \\
x_{0} \in B_{0} \cap W^{s}\left(y_{0}\right) \neq \varnothing .
\end{gathered}
$$

As $W^{u}(\Lambda) \pitchfork W^{s}\left(\sigma\left(f^{n_{0}}\left(y_{0}\right)\right)\right)$ at an homoclinic point that we call $p_{0}$, by the lambda lemma there exists a point $\bar{x}_{0} \in W^{s}\left(\sigma\left(f^{n_{0}}\left(y_{0}\right)\right)\right) \cap V_{\Gamma}$ such that $f^{-n_{0}}\left(\bar{x}_{0}\right) \in B_{0}$ if $n_{0} \geq n^{*}$.

(2) By continuity, there exists a ball $V_{0} \subset V_{\Gamma}$ centered at $\bar{x}_{0}$ such that

$$
f^{-n_{0}}\left(V_{0}\right) \subset B_{0} \subset B_{\delta}\left(y_{0}\right) \subset V_{\Lambda} .
$$

Now we proceed with the second step of the induction procedure:

(1) By the definition of $n^{*}$, as $\bar{x}_{0} \in W^{s}\left(\sigma\left(f^{n_{0}}\left(y_{0}\right)\right)\right) \cap V_{\Gamma}$, as $k^{+} \geq n^{*}$, we know that

$$
f^{k^{+}}\left(\bar{x}_{0}\right) \in W^{s}\left(f^{k^{+}}\left(\sigma\left(f^{n_{0}}\left(y_{0}\right)\right)\right)\right) \cap B_{\delta}\left(f^{k^{+}}\left(\sigma\left(f^{n_{0}}\left(y_{0}\right)\right)\right)\right) \subset V_{\Lambda} .
$$

(2) By continuity, there is a ball $U_{1}$ centered at $f^{k^{+}}\left(\bar{x}_{0}\right)$ such that

$$
\begin{gathered}
U_{1} \subset B_{\delta}\left(f^{k^{+}}\left(\sigma\left(f^{n_{0}}\left(y_{0}\right)\right)\right)\right) \subset V_{\Lambda}, \\
f^{k^{+}}\left(\bar{x}_{0}\right) \in U_{1} \cap W^{s}\left(f^{k^{+}}\left(\sigma\left(f^{n_{0}}\left(y_{0}\right)\right)\right)\right), \\
f^{-k^{+}}\left(U_{1}\right) \subset V_{0} .
\end{gathered}
$$

(3) Recall that $y_{1}=f^{m_{0}}\left(\sigma\left(f^{n_{0}}\left(y_{0}\right)\right)\right)$, and therefore $f^{k^{+}}\left(\sigma\left(f^{n_{0}}\left(y_{0}\right)\right)\right)=$ $f^{k^{+}-m_{0}}\left(y_{1}\right)$.

(4) The next step is to apply the lambda lemma. Now $p^{--}=f^{n_{1}}\left(y_{1}\right), p^{++}=$ $\sigma\left(f^{n_{1}}\left(y_{1}\right)\right)$, and $k^{\prime-}=n_{1}$. As $W^{u}(\Lambda)$ intersects $W^{s}\left(\sigma\left(f^{n_{1}}\left(y_{1}\right)\right)\right)$ transversally at an homoclinic point $p_{1}$, if we take $n_{1}>k^{+} \geq n^{*}$ and $m_{0}>$ $m_{0}^{*}$, where $m_{0}^{*}$ is the value $m^{*}$ given in the general step and depends on the size of $U_{1}$ and therefore on $n_{0}$, one has that $n_{1}+m_{0}-k^{+}>k^{+}+$ $m_{0}-k^{+}=m_{0}>m_{0}^{*}$, and there exists $x_{1} \in W^{s}\left(\sigma\left(f^{n_{1}}\left(y_{1}\right)\right)\right)$ and a ball $V_{1}$ centered at $x_{1}$ such that

$$
\begin{gathered}
f^{-n_{1}}\left(x_{1}\right) \in f^{-n_{1}}\left(V_{1}\right) \subset B_{\delta}\left(y_{1}\right), \\
f^{-\left(n_{1}+m_{0}-k^{+}\right)}\left(x_{1}\right) \in f^{-\left(n_{1}+m_{0}-k^{+}\right)}\left(V_{1}\right) \subset U_{1} .
\end{gathered}
$$




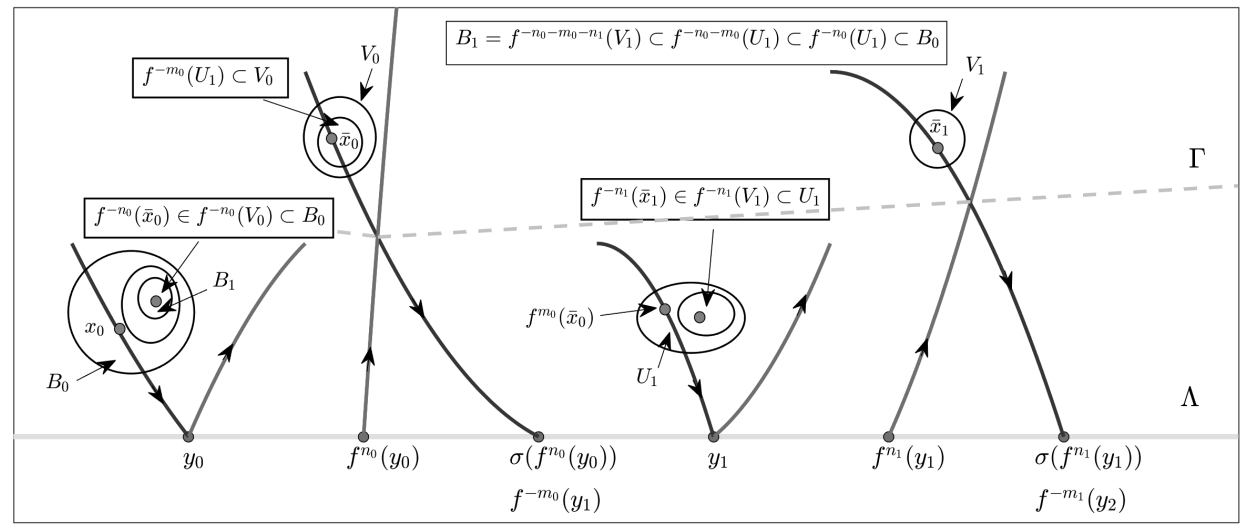

FIGURE 5.2. The construction in the obstruction argument.

(5) If we now take $B_{1}=f^{-\left(n_{0}+n_{1}+m_{0}\right)}\left(V_{1}\right)$, we have, using (5.24), 5.22), and (5.21), that

$$
\begin{aligned}
B_{1}=f^{-\left(n_{0}+n_{1}+m_{0}\right)}\left(V_{1}\right) & =f^{-n_{0}-k^{+}} \circ f^{-n_{1}-m_{0}+k^{+}}\left(V_{1}\right) \\
& \subset f^{-n_{0}-k^{+}}\left(U_{1}\right) \subset f^{-n_{0}}\left(V_{0}\right) \subset B_{0} .
\end{aligned}
$$

Moreover, if we take $z_{0} \in B_{1}$ it satisfies, by (5.25) and (5.23) and using that $B_{0} \subset B_{\delta}\left(y_{0}\right)$,

$$
\begin{aligned}
z_{0} & \in B_{\delta}\left(y_{0}\right), \\
f^{n_{0}+m_{0}}\left(z_{0}\right) & \in f^{-n_{1}}\left(V_{1}\right) \subset B_{\delta}\left(y_{1}\right) .
\end{aligned}
$$

A schematic illustration of the first step in the induction construction above is shown in Figure 5.2 .

Once we have done the two first steps, we can proceed with the general induction step.

Assume we have built the sequence $\bar{x}_{i} \in W^{s}\left(\sigma\left(f^{n_{i}}\left(y_{i}\right)\right)\right) \cap V_{\Gamma}$, a ball $V_{i} \subset V_{\Lambda}$ centered at $\bar{x}_{i}, i=0, \ldots, j$, and $U_{i+1}$ a ball centered at $f^{k^{+}}\left(\bar{x}_{i}\right)$ for $n_{i}>k^{+} \geq$ $n^{*}$, and $m_{i} \geq m_{i}^{*}$ for $i=0, \ldots, j$, with the following properties:

- $f^{-n_{i}}\left(V_{i}\right) \subset B_{\delta}\left(y_{i}\right)$,

- $f^{-\left(n_{i}+m_{i-1}-k^{+}\right)}\left(V_{i}\right) \subset U_{i}$,

- $U_{i+1} \subset B_{\delta}\left(f^{k^{+}}\left(\sigma\left(f^{n_{i}}\left(y_{i}\right)\right)\right)\right)$,

- $f^{k^{+}}\left(\bar{x}_{i}\right) \in U_{i+1}$,

- $f^{-k^{+}}\left(U_{i+1}\right) \subset V_{i}$.

We also assume that we have $\bar{x}_{j+1} \in V_{j+1} \cap W^{s}\left(\sigma\left(f^{n_{j+1}}\left(y_{j+1}\right)\right)\right)$ such that

- $f^{-n_{j+1}}\left(V_{j+1}\right) \subset B_{\delta}\left(y_{j+1}\right)$,

- $f^{-\left(n_{j+1}+m_{j}-k^{+}\right)}\left(V_{j+1}\right) \subset U_{j+1}$. 
Let

$$
B_{j+1}=f^{-n_{j+1}} \circ f^{-\sum_{i=0}^{j} m_{i}+n_{i}}\left(V_{j+1}\right),
$$

and we have that $B_{j+1} \subset B_{j} \subset B_{j-1} \subset \cdots \subset B_{0}$.

To proceed, first we look for a ball $U_{j+2}$ centered at $f^{k^{+}}\left(\bar{x}_{j+1}\right)$ such that

$$
\begin{gathered}
U_{j+2} \subset B_{\delta}\left(f^{k^{+}}\left(\sigma\left(f^{n_{j+1}}\left(y_{j+1}\right)\right)\right)\right), \\
f^{k^{+}}\left(\bar{x}_{j+1}\right) \in U_{j+2} \cap W^{s}\left(f^{k^{+}} \sigma\left(f^{n_{j+1}}\left(y_{j+1}\right)\right)\right), \\
f^{-k^{+}}\left(U_{j+2}\right) \subset V_{j+1} .
\end{gathered}
$$

The value of $k^{+}$and $n^{*}$ are fixed, but the size of $U_{j+2}$ depends on the size of $V_{j+1}$ and therefore on the previous steps. Then, applying the lambda lemma, using $y_{j+2}=f^{m_{j+1}}\left(\sigma\left(f^{n_{j+1}}\left(y_{j+1}\right)\right)\right.$ and $\left.W^{u}(\Lambda)\right) \pitchfork W^{s}\left(\sigma\left(f^{n_{j+2}}\left(y_{j+2}\right)\right)\right)$ at a point $p_{j+2}$, we will find $\bar{x}_{j+2} \in W^{s}\left(\sigma\left(f^{n_{j+2}}\left(y_{j+2}\right)\right)\right)$ and a ball $V_{j+2} \subset V_{\Gamma}$ centered at $\bar{x}_{j+2}$ such that, if $n_{j+2}>k^{+} \geq n^{*}$ and $m_{j+1}>m_{j+1}^{*}$, then

- $f^{-n_{j+2}}\left(V_{j+2}\right) \subset B_{\delta}\left(y_{j+2}\right)$,

- $f^{-\left(n_{j+2}+m_{j+1}-k^{+}\right)}\left(V_{j+2}\right) \subset U_{j+2}$.

Observe that the value $m_{j+1}^{*}$ is the general value $m^{*}$ that now depends on the size of $U_{j+2}$, and therefore of all the previous steps.

Finally, define

$$
B_{j+2}=f^{-\sum_{k=0}^{j} m_{k}+n_{k}} \circ f^{-n_{j+2}-m_{j+1}-n_{j+1}}\left(V_{j+2}\right) .
$$

Then we have

$$
\begin{aligned}
f^{-n_{j+2}-m_{j+1}-n_{j+1}}\left(V_{j+2}\right) & =f^{-n_{j+1}-n^{*}} \circ f^{-n_{j+2}-m_{j+1}+n^{*}}\left(V_{j+2}\right) \\
& \subset f^{-n_{j+1}-n^{*}}\left(U_{j+2}\right) \subset f^{-n_{j+1}}\left(V_{j+1}\right) .
\end{aligned}
$$

Therefore

$$
B_{j+2} \subset f^{-\sum_{k=0}^{j} m_{k}+n_{k}} \circ f^{-n_{j+1}}\left(V_{j+1}\right)=B_{j+1} .
$$

This finishes the induction procedure. Observe that if $z_{0} \in \bigcap_{0 \leq i \leq j} B_{j}$ and we consider the orbit $z_{i+1}=f^{m_{i}+m_{i}}\left(z_{i}\right)$, we have that

- $z_{0} \in B_{0} \subset B_{\delta}\left(y_{0}\right)$, and

- for all $i=0, \ldots, j, z_{0} \in B_{i}$, and therefore, by the definition of $B_{i}$,

$$
z_{i}=f^{n_{0}+m_{0}+\cdots+n_{i-1}+m_{i-1}}\left(z_{0}\right) \in f^{-n_{i}}\left(V_{i}\right) \subset B_{\delta}\left(y_{i}\right) .
$$

To finish the proof we just point out that the definition of $m_{j+1}^{*}$ depends on the size of the balls $U_{j+1}$ but not on the points $y_{j}$ themselves. Therefore, if another pseudo-orbit is given with the same indexes $n_{i}$ and $m_{j}$, the same choices of $n^{*}$ and $m_{i}^{*}$ will work. 


\subsubsection{Remarks}

Remark 5.1. In the proof of Lemma 3.1 given in Section 5.1.1, we have constructed windows $W_{i}^{-}, W_{i}^{+}, W_{i+1}^{-}$in $V_{\Lambda}$ so that $f^{t}\left(W_{i}^{+}\right) \subseteq V_{\Lambda}, 0 \leq t \leq m_{i}-k^{+}$, so the corresponding segment of the shadowing orbit of $z_{i}$ stays in $V_{\Lambda}$ for this entire time. Thus, the construction in the proof of the lemma enables one to find shadowing orbits that stay close to $\Lambda$ for any sufficiently long time intervals between two consecutive homoclinic excursions.

Remark 5.2. Lemma 3.1 provides a true forward orbit that shadows a given forward pseudo-orbit. The current proofs do not allow for immediately extending this result for bi-infinite orbits. We remark that there is no assumption on the inner dynamics given by $f_{\mid \Lambda}$. In the proof given in Section 5.1.1, the alignment of windows in the center directions was achieved by defining, at each step of the construction, the center component of $W_{i+1}^{-}$as a ball inside some forward image of the centercomponent of $W_{i}^{+}$. Thus, the consecutive balls in the center direction can get smaller and smaller in size as $i$ increases. So if we try to continue the procedure in backwards time, the center-components of the windows $W_{i}^{+}, i \leq 0$, may get bigger and bigger in size. Thus, we may lose control on the shadowing trajectory; that is, the resulting shadowing orbit does not follow $\delta$-closely the prescribed pseudo-orbit.

Remark 5.3. Statements related to Lemma 3.1 appear in [39, 40,60]. The main difference is that the statements in these papers assume certain geometric conditions on the inner dynamics.

There is also a related version of the shadowing lemma in [52], but only for finite pseudo-orbits; moreover, those pseudo-orbits are subject to certain conditions that are very different from ours.

Remark 5.4. It is interesting to note that the geometric proof of Lemma 3.1 given in Section 5.1.2 works in infinite dimensions. One only needs to substitute the compactness assumptions by the assumption that the regularity of the maps-and hence of the manifolds-are uniform.

Indeed, infinite-dimensional versions of the theory of normally hyperbolic manifolds appear in [4, 86]. An infinite-dimensional version of the inclination lemma appears in [29]. Note also that the nested-balls arguments also work in infinite dimensions when the space we consider is reflexive (or the dual of Banach space). It suffices to note that by the Banach-Alaoglu theorem, balls are compact in the weak-* topology.

\subsection{Proof of Theorem 3.6}

Denote by $\mu$ the measure referred to in the statement of the theorem, which is absolutely continuous with respect to the Lebesgue measure on $\Lambda$. Then $f$ preserves $\mu$, and $\sigma$ takes positive measure sets onto positive measure sets.

Choose a small open disk $B_{0}$ of $x_{0}$ in $\Lambda$, with $B_{0} \subseteq \mathcal{U}$ such that $B_{i}:=$ $\sigma^{i}\left(B_{0}\right) \subseteq \mathcal{U}$ and $\operatorname{diam}\left(B_{i}\right) \leq \delta / 2$ for all $i=0, \ldots, n$. For the given pseudo-orbit $\left\{x_{i}\right\}$ of $\sigma$, with $x_{i+1}=\sigma\left(x_{i}\right)$, we have that $x_{i} \in B_{i}$ for all $i$. We will use Poincaré 
recurrence to produce a new pseudo-orbit $\left\{y_{i}\right\}$, with $y_{i+1}=f^{m_{i}} \circ \sigma \circ f^{n_{i}}\left(y_{i}\right)$, where $m_{i}$ and $n_{i}$ are as in Lemma 3.1 such that $y_{i} \in B_{i}$ for all $i$, and hence $d\left(y_{i}, x_{i}\right) \leq \delta / 2$. Invoking Lemma 3.1 will provide us with a true orbit $\left\{z_{i}\right\}$ with $z_{i+1}=f^{m_{i}+n_{i}}\left(z_{i}\right)$ such that $d\left(z_{i}, y_{i}\right) \leq \delta / 2$, hence $d\left(z_{i}, x_{i}\right)<\delta$.

We first establish some basic facts about recurrent points.

\subsubsection{First Recurrence Property}

For an open set $B \subseteq \mathcal{U} \subseteq \Lambda$, a subset $A \subseteq B$ of positive measure in $B$, and $k^{*}>0$, we define

$$
P^{k^{*}}(A, B)=\left\{x \in A \mid\left(f^{k^{*}}\right)^{t}(x) \in B \text { for some } t \geq 1\right\} .
$$

The set $P^{k^{*}}(A, B) \subset A$ consists of the recurrent points of $A$ that return to $B$ under some positive iteration of $f^{k^{*}}$. Since $\mu$-a.e. point in $\mathcal{U}$ is recurrent, and $B \subseteq \mathcal{U}$, Poincaré recurrence for the map $f^{k^{*}}$ implies that $P^{k^{*}}(A, B) \subseteq A$ has full measure in $A$, and hence is of positive measure itself.

For each $x \in P^{k^{*}}(A, B)$ let $t_{\min }(x)$ be the smallest positive integer $t \geq 1$ with $\left(f^{k^{*}}\right)^{t}(x) \in B$. Let

$$
\Theta=\left\{\tau \geq 1 \mid \exists x \in P^{k^{*}}(A, B) \text { s.t. } t_{\min }(y)=\tau\right\}
$$

be the set of the return times to $B$. For each $\tau \in \Theta$, let

$$
P_{\tau}^{k^{*}}(A, B)=\left\{x \in P^{k^{*}}(A, B) \mid t_{\min }(y)=\tau\right\}
$$

be the set of points with a prescribed return time $\tau \in \Theta$ under $f^{k^{*}}$.

Since $P_{k^{*}}(A, B)=\bigcup_{\tau \geq 1} P_{\tau}^{k^{*}}(A, B)$, with the sets $P_{\tau}^{k^{*}}(A, B)$ mutually disjoint, there exists $\tau^{*} \geq 1$ such that $\mu\left(P_{\tau^{*}}^{k^{*}}(A, B)\right)>0$. Since $f^{k^{*}}$ is area preserving, $\mu\left(f^{k^{*} \tau^{*}}\left(P_{\tau^{*}}^{k^{*}}(A, B)\right)=\mu\left(P_{\tau^{*}}^{k^{*}}(A, B)\right)>0\right.$.

Thus, every point in $P_{\tau^{*}}^{k^{*}}(A, B) \subseteq A \subseteq B$ will return to a point in $B$ under $f^{k^{*} \tau^{*}}$. The set

$$
Q_{\tau^{*}}^{k^{*}}(B, A):=f^{k^{*} \tau^{*}}\left(P_{\tau^{*}}^{k^{*}}(A, B)\right) \subseteq B
$$

has positive measure in $B$. In terms of $f$, every point in $P_{\tau^{*}}^{k^{*}}(A, B) \subseteq A \subseteq B$ will return to a point in $Q_{\tau^{*}}^{k^{*}}(A, B) \subseteq B$ in exactly $k^{*} \tau^{*} \geq k^{*}$ iterates.

\subsubsection{Second Recurrence Property}

Consider now two open sets $B \subseteq \mathcal{U}$ and $B^{\prime}=\sigma(B) \subseteq \mathcal{U}$. Let $A$ be a subset of $B$ of positive measure. By the above, $P_{\tau^{*}}^{k^{*}}(A, B)$ and $Q_{\tau^{*}}^{k^{*}}(A, B)$ are positive measure subsets of $B$. Since the scattering map $\sigma$ sends positive measure sets onto positive measure sets, it follows that

$$
A^{\prime}:=\sigma\left(Q_{\tau^{*}}^{k^{*}}(A, B)\right) \subset B^{\prime}
$$

is a positive measure subset of $B^{\prime}$. 


\subsubsection{Inductive Construction of Pseudo-orbits}

Starting with $B_{0}$, we construct inductively a nested sequence of subsets $\Sigma_{i} \subset$ $B_{0}$ of positive measure of $B_{0}$ such that each set is carried onto a positive measure subset of $B_{i}, i=1, \ldots, n$, via successive applications of some large powers of $f$ interspersed with applications of $\sigma$.

Use Lemma 3.1 for $\delta / 2$, and consider the value $n^{*}$ depending on $\delta / 2$ as provided by this lemma. Let $A_{0}:=B_{0}$, let $\tau_{0} \geq 1$ such that $P_{\tau_{0}}^{n^{*}}\left(A_{0}, B_{0}\right) \subset A_{0}$ (see 5.27) has positive measure, and

$$
\Sigma_{0}:=P_{\tau_{0}}^{n^{*}}\left(A_{0}, B_{0}\right) \subseteq A_{0} .
$$

Consider the set $Q_{\tau_{0}}^{n^{*}}\left(A_{0}, B_{0}\right) \subseteq B_{0}$ (see (5.28), which has positive measure. Then consider the set $A_{1}^{\prime}:=\sigma\left(Q_{\tau_{0}}^{n^{*}}\left(A_{0}, B_{0}\right)\right) \subseteq B_{1}$ (see 5.29$)$ ), which has positive measure in $B_{1}$. Let $n_{0}:=n^{*} \tau_{0}$ and consider the value $m_{0}^{*}=m_{0}^{*}\left(n_{0}\right)$ given by Lemma 3.1 for $\delta / 2$. There exists $\tau_{0}^{\prime} \geq 1$ such that the set

$$
P_{\tau_{0}^{\prime}}^{m_{0}^{*}}\left(A_{1}^{\prime}, B_{1}\right) \subseteq A_{1}^{\prime} \subseteq B_{1}
$$

(see (5.27) ) has positive measure. Then the set $Q_{\tau_{0}^{\prime}}^{m_{0}^{*}}\left(A_{1}^{\prime}, B_{1}\right) \subseteq B_{1}$ (see 5.28) also has positive measure in $B_{1}$.

Each point $y_{1} \in Q_{\tau_{0}^{\prime}}^{m_{0}^{*}}\left(A_{1}^{\prime}, B_{1}\right)$ is of the form $y_{1}=f^{m_{0}^{*} \tau_{0}^{\prime}}\left(x^{\prime}\right)$, for some $x^{\prime} \in$ $P_{\tau_{0}^{\prime}}^{m^{*}}\left(A_{1}^{\prime}, B_{1}\right)$ and $\tau_{0}^{\prime} \geq 1$; each such $x^{\prime}$ is of the form $x^{\prime}=\sigma(x)$ for some $x \in$ $Q_{\tau_{0}}^{n^{*}}\left(A_{0}, B_{0}\right)$; and each such $x$ is of the form $x=f^{n^{*} \tau_{0}}\left(y_{0}\right)$ for some $y_{0} \in$ $P_{\tau_{0}}^{n^{*}}\left(A_{0}, B_{0}\right)=\Sigma_{0}$ and $\tau_{0} \geq 1$. Denote $m_{0}:=m_{0}^{*} \tau_{0}^{\prime}$ and $A_{1}:=Q_{\tau_{0}^{\prime}}^{m_{0}^{*}}\left(A_{1}^{\prime}, B_{1}\right) \subseteq$ $B_{1}$. Thus, each $y_{1} \in A_{1}$ can be written as

$$
y_{1}=f^{m_{0}} \circ \sigma \circ f^{n_{0}}\left(y_{0}\right)
$$

for some $y_{0} \in \Sigma_{0}, n_{0} \geq n^{*}$, and $m_{0} \geq m^{*}$, where $m_{0}=m_{0}^{*} \tau_{0}^{\prime}$ and $n_{0}=n^{*} \tau_{0}$.

Denote by $\Sigma_{1}$ the set of points $y_{0} \in \Sigma_{0}$ that correspond, via (5.30), to some point $y_{1} \in A_{1}$. We obviously have $\Sigma_{1} \subseteq \Sigma_{0}$. The preliminary facts established above show that $\Sigma_{1}$ is a positive measure subset of $B_{0}$.

Assume that at the $j^{\text {th }}$ step we have constructed a subset $A_{j} \subseteq B_{j}$, which has positive measure in $B_{j}$, such that each point $y_{j} \in A_{j}$ is of the form

$$
y_{j}=f^{m_{j-1}} \circ \sigma \circ f^{n_{j-1}} \circ \cdots \circ f^{m_{0}} \circ \sigma \circ f^{n_{0}}\left(y_{0}\right),
$$

for some $y_{0} \in A_{0} \subset B_{0}$, with $n_{0} \geq n^{*}, \ldots, n_{j-1} \geq n^{*}$ and $m_{0} \geq m_{0}^{*}, \ldots, m_{j-1}$ $\geq m_{j-1}^{*}$, where $n^{*}$ and the $m_{k}^{*}$ 's are as in Lemma 3.1. Let $\Sigma_{j}$ be the set of points $y_{0}$ for which the corresponding $y_{j}$ given by (5.31) is in $A_{j}$. We assume that $\Sigma_{j} \subseteq$ $\Sigma_{j-1} \subseteq \cdots \subseteq \Sigma_{0}$, and that $\Sigma_{j}$ is a positive measure subset of $B_{0}$.

It follows from the above preliminaries that, for some $\tau_{j} \geq 1$, the sets

$$
P_{\tau_{j}}^{n^{*}}\left(A_{j}, B_{j}\right) \subseteq B_{j} \quad \text { and } \quad Q_{\tau_{j}}^{n^{*}}\left(A_{j}, B_{j}\right) \subseteq B_{j}=f^{n^{*} \tau_{j}}\left(P_{\tau_{j}}^{n^{*}}\left(A_{j}, B_{j}\right)\right)
$$


have positive measure. Each point $y \in P_{\tau_{j}}^{n^{*}}\left(A_{j}, B_{j}\right)$ returns to a point in

$$
Q_{\tau_{j}}^{n^{*}}\left(A_{j}, B_{j}\right) \subseteq B_{j}
$$

after exactly $n^{*} \tau_{j}$ iterates of $f$. Denote $n_{j}:=n^{*} \tau_{j}$. Since $\sigma$ is measure preserving, the set $A_{j+1}^{\prime}:=\sigma\left(Q_{\tau_{j}}^{n^{*}}\left(A_{j}, B_{j}\right)\right) \subset B_{j+1}$ has positive measure in $B_{j+1}$. Let $m_{j}^{*}$, depending on $\delta / 2$ and on $n_{0}, \ldots, n_{j}, m_{0}, \ldots, m_{j-1}$, be as in the Lemma 3.1 . There exists $\tau_{j}^{\prime} \geq 1$ such that

$$
P_{\tau_{j}^{\prime}}^{m_{j}^{*}}\left(A_{j+1}^{\prime}, B_{j+1}\right) \subseteq A_{j+1}^{\prime} \subseteq B_{j+1}
$$

and $Q_{\tau_{j}^{\prime}}^{m_{j}^{*}}\left(A_{j+1}^{\prime}, B_{j+1}\right) \subseteq B_{j+1}$ have positive measure. Each point

$$
y \in P_{\tau_{j}^{\prime}}^{m_{j}^{*}}\left(A_{j+1}^{\prime}, B_{j+1}\right)
$$

returns to a point in $Q_{\tau_{j}^{\prime}}^{m_{j}^{*}}\left(A_{j+1}^{\prime}, B_{j+1}\right) \subseteq B_{j+1}$ after exactly $m_{j}^{*} \tau_{j}^{\prime}$ iterates of $f$. Denote $A_{j+1}=Q_{\tau_{j}^{\prime}}^{m_{j}^{*}}\left(A_{j+1}^{\prime}, B_{j+1}\right)$, which is of positive measure. Then each point $y_{j+1} \in A_{j+1}$ is of the form

$$
y_{j+1}=f^{m_{j}} \circ \sigma \circ f^{n_{j}}\left(y_{j}\right)
$$

for some $y_{j} \in A_{j}$, where $n_{j}=n^{*} \tau_{j} \geq n^{*}$ and $m_{j}=m_{j}^{*} \tau_{j}^{\prime} \geq m_{j}^{*}$, with $\tau_{j}, \tau_{j}^{\prime} \geq 1$.

Since $y_{j}$ is of the form (5.31), then

$$
y_{j+1}=f^{m_{j}} \circ \sigma \circ f^{n_{j}} \circ \cdots \circ f^{m_{0}} \circ \sigma \circ f^{n_{0}}\left(y_{0}\right)
$$

for some $y_{0} \in \Sigma_{0}$, with $n_{0} \geq n^{*}, \ldots, n_{j-1} \geq n^{*}$ and $m_{0} \geq m_{0}^{*}, \ldots, m_{j} \geq m_{j}^{*}$. Denoting by $\Sigma_{j+1}$ the set of points $y_{0} \in \Sigma_{0}$ that yield points $y_{j+1}$ given by (5.34), we obtain that $\Sigma_{j+1} \subseteq \Sigma_{j}$ is of positive measure. This completes the induction step.

\subsubsection{Shadowing of Pseudo-orbits}

At the $n^{\text {th }}$ step we obtain a nested sequence of sets $\Sigma_{0} \supseteq \Sigma_{1} \supseteq \cdots \supseteq \Sigma_{n}$ such that each set $\Sigma_{j}, j=0, \ldots, n$, has positive measure in $B_{0}$. Each point $y_{0} \in \Sigma_{n}$ generates a pseudo-orbit of the form

$$
y_{j+1}=f^{m_{j}} \circ \sigma \circ f^{n_{j}}\left(y_{j}\right)
$$

for $j=0, \ldots, n-1$, where $n_{j}$ and $m_{j}$ are as in Lemma 3.1. By construction, each point $y_{j}$ is inside $B_{j}$, hence $d\left(y_{j}, x_{j}\right)<\delta / 2$. Then Lemma 3.1 provides the existence of an orbit $\left\{z_{j}\right\}_{j=0, \ldots, n}$ with $z_{j+1}=f^{m_{j}+n_{j}}\left(z_{j}\right)$ such that $d\left(z_{j}, y_{j}\right)<$ $\delta / 2$. Hence $d\left(z_{j}, x_{j}\right)<\delta$ for all $j$.

Remark 5.5. In the proof of Theorem 3.6, instead of using the Poincare recurrence theorem we can use the nonwandering property given by Proposition 2.4. Starting with $B_{0}^{\prime}=B_{0}$, there exists $n_{0} \geq n^{*}$ such that $f^{n_{0}}\left(B_{0}^{\prime}\right) \cap B_{0}^{\prime} \neq \varnothing$. The set 
$\widetilde{B}_{0}^{\prime}=f^{n_{0}}\left(B_{0}^{\prime}\right) \cap B_{0}^{\prime}$ is an open set in $B_{0}^{\prime}$, and $\sigma\left(\widetilde{B}_{0}^{\prime}\right) \subseteq B_{1}$. There exists $m_{0} \geq m_{0}^{*}$ such that $f^{m_{0}}\left(\sigma\left(\widetilde{B}_{0}^{\prime}\right)\right) \cap \sigma\left(\widetilde{B}_{0}^{\prime}\right) \neq \varnothing$. The set $B_{1}^{\prime}:=\sigma\left(f^{m_{0}}\left(\sigma\left(\widetilde{B}_{0}^{\prime}\right)\right) \cap \sigma\left(\widetilde{B}_{0}^{\prime}\right)\right)$ is an open set in $B_{1}$. The construction can be continued recursively as before. Given the open set $B_{j}^{\prime} \subseteq B_{j}$ obtained at the end of the $(j-1)^{\text {th }}$ step, at step $j$ we construct $\widetilde{B}_{j}^{\prime}=f^{n_{j}}\left(B_{j}^{\prime}\right) \cap B_{j}^{\prime}$ for $n_{j} \geq n^{*}, \sigma\left(\widetilde{B}_{j}^{\prime}\right) \subseteq B_{j+1}$, and $B_{j+1}^{\prime}:=$ $f^{m_{j}}\left(\sigma\left(\widetilde{B}_{j}^{\prime}\right)\right) \cap \sigma\left(\widetilde{B}_{j}^{\prime}\right) \neq \varnothing$ for $m_{j} \geq m_{j}^{*}$. The initial points $y_{0} \in B_{0}$ that generate pseudo-orbits of the form (5.34) for $j=0, \ldots, n-1$ form an open set $\Sigma_{n} \subseteq B_{0}$.

This approach yields explicit estimates of the return times to $B_{j}^{\prime}$ and $\sigma\left(\tilde{B}_{j}^{\prime}\right)$, given by $O\left(1 / \mu\left(B_{j}^{\prime}\right)\right)$ and $O\left(1 / \mu\left(\sigma\left(\widetilde{B}_{j}^{\prime}\right)\right)\right)$, respectively. These estimates on the return time, together with the data on the hyperbolic expansion/contraction rates and on the angle of intersection between the stable and unstable manifolds (see Remark 3.3 can be used to obtain explicit—but far from optimal—estimates on the diffusion time.

\subsection{Proof of Theorem 3.11}

We notice that 3.2 is reminiscent of the forward Euler method with step $\mu(\varepsilon)$ for ordinary differential equations.

As $J \nabla S\left(\tilde{x}_{0}\right) \neq 0$ at some point $\tilde{x}_{0} \in U \subset \Lambda_{0}$, we know that the solution

$$
\frac{d}{d t} \tilde{\gamma}(t)=J \nabla S \circ \tilde{\gamma}(t)
$$

with $\tilde{\gamma}(0)=\tilde{x}_{0}$ is not a constant solution. Let's denote $\tilde{\gamma}(t)=\phi\left(t, \tilde{x}_{0}\right)$ where $\phi(t, x)$ is the flow of (5.35). Consider $n=\left\lfloor\mu^{-1}\right\rfloor$, where $\mu=\mu(\varepsilon)$ is the parameter that appears in $(3.2)$, and $\lfloor\cdot\rfloor$ denotes the floor function. Define two sequences:

$$
\tilde{y}_{i}=\tilde{\gamma}\left(t_{i}\right)=\phi\left(\Delta t, \tilde{y}_{i-1}\right), \quad \tilde{x}_{i}=\tilde{\sigma}_{\varepsilon}\left(\tilde{x}_{i-1}\right), \quad i=1,2, \ldots, n, \tilde{x}_{0}=\tilde{y}_{0},
$$

where $t_{i}=i \mu$ and $\Delta t=\mu$. We will use two facts.

On one hand, if we apply Gronwall's lemma to the vector field (5.35), there exists a constant $K_{1}>0$ such that

$$
\left\|\phi(\Delta t, \tilde{y})-\phi\left(\Delta t, \tilde{y}^{\prime}\right)\right\| \leq \mathrm{e}^{K_{1} \mu}\left\|\tilde{y}-\tilde{y}^{\prime}\right\| \quad \text { for } \tilde{y}, \tilde{y}^{\prime} \in \mathcal{U}_{\tilde{\gamma}} .
$$

On the other hand, also by (3.2), calling

$$
\tilde{g}(\mu)=|g(\mu)| / \mu=o(1),
$$

there exists a constant $K_{2}>0$ that is independent of $\mu$ and $\varepsilon$ such that

$$
\left\|\tilde{\sigma}_{\varepsilon}(\tilde{x})-\phi(\Delta t, \tilde{x})\right\| \leq K_{2} \mu(\mu+\tilde{g}(\mu)) \text { for } \tilde{x} \in \mathcal{U}_{\tilde{\gamma}} .
$$

Now one easily obtains that, by 5.37,

$$
\left\|\tilde{x}_{1}-\tilde{y}_{1}\right\|=\left\|\tilde{\sigma}_{\varepsilon}\left(\tilde{x}_{0}\right)-\phi\left(\mu, \tilde{x}_{0}\right)\right\| \leq K_{2} \mu(\mu+\tilde{g}(\mu)),
$$

and, consequently, $\tilde{x}_{1} \in \mathcal{U}_{\tilde{\gamma}}$. 
Now, using again (5.36) and (5.37), we get

$$
\begin{aligned}
\left\|\tilde{x}_{2}-\tilde{y}_{2}\right\| & =\left\|\tilde{\sigma}_{\varepsilon}\left(\tilde{x}_{1}\right)-\phi\left(\mu, \tilde{y}_{1}\right)\right\| \\
& \leq\left\|\tilde{\sigma}_{\varepsilon}\left(\tilde{x}_{1}\right)-\phi\left(\mu, \tilde{x}_{1}\right)\right\|+\left\|\phi\left(\mu, \tilde{x}_{1}\right)-\phi\left(\mu, \tilde{y}_{1}\right)\right\| \\
& \leq K_{2} \mu(\mu+\tilde{g}(\mu))+\mathrm{e}^{K_{1} \mu}\left\|\tilde{x}_{1}-\tilde{y}_{1}\right\| \leq K_{2} \mu(\mu+\tilde{g}(\mu))(1+c),
\end{aligned}
$$

where we denote $c=\mathrm{e}^{K_{1} \mu}>1$.

Consequently, $\tilde{x}_{2} \in \mathcal{U}_{\tilde{\gamma}}$. Now we proceed by induction. We assume that, for some $0 \leq i \leq n$, one has that

$$
\left\|\tilde{x}_{i}-\tilde{y}_{i}\right\| \leq K_{2} \mu(\mu+\tilde{g}(\mu))\left(1+c+c^{2}+\cdots+c^{i-1}\right) .
$$

Using again (5.36) and 5.37) we obtain

$$
\begin{aligned}
\left\|\tilde{x}_{i+1}-\tilde{y}_{i+1}\right\| & =\left\|\tilde{\sigma}_{\varepsilon}\left(\tilde{x}_{i}\right)-\phi\left(\mu, \tilde{y}_{i}\right)\right\| \\
& \leq\left\|\tilde{\sigma}_{\varepsilon}\left(\tilde{x}_{i}\right)-\phi\left(\mu, \tilde{x}_{i}\right)\right\|+\left\|\phi\left(\mu, \tilde{x}_{i}\right)-\phi\left(\mu, \tilde{y}_{i}\right)\right\| \\
& \leq K_{2} \mu(\mu+\tilde{g}(\mu))+\mathrm{e}^{K_{1} \mu}\left\|\tilde{x}_{i}-\tilde{y}_{i}\right\| \\
& \leq K_{2} \mu(\mu+\tilde{g}(\mu))\left(1+c+c^{2}+\cdots+c^{i}\right) .
\end{aligned}
$$

Therefore, using that $c=\mathrm{e}^{K_{1} \mu}$, that $c-1=\mathrm{e}^{K_{1} \mu}-1 \geq K_{1} \mu$, and that $n=$ $\left\lfloor\mu^{-1}\right\rfloor$, for $i=0,1, \ldots, n$, we have that

$$
\begin{aligned}
\left\|\tilde{x}_{i}-\tilde{y}_{i}\right\| \leq K_{2} \mu(\mu+\widetilde{g}(\mu)) \frac{c^{i}-1}{c-1} & \leq \frac{K_{2}}{K_{1}}(\mu+\widetilde{g}(\mu)) \mathrm{e}^{i K_{1} \mu} \\
& \leq \frac{K_{2}}{K_{1}}(\mu+\widetilde{g}(\mu)) \mathrm{e}^{K_{1}}
\end{aligned}
$$

As $\mu=\mu(\varepsilon)=o(\varepsilon)$, there exists $\varepsilon_{1}$ such that if $0<\varepsilon \leq \varepsilon_{1}$, we obtain that the sequence $\tilde{x}_{i}$ of the scattering map is also in $\mathcal{U}_{\tilde{\gamma}}$ and is $(\mu+\tilde{g}(\mu))$-close to the orbit $\tilde{\gamma}$ :

$\tilde{x}_{i+1}=\tilde{\sigma}_{\varepsilon}\left(\tilde{x}_{i}\right) \in \mathcal{U}_{\tilde{\gamma}} \subset \Lambda, \quad d\left(\widetilde{\gamma}\left(t_{i}\right), \tilde{x}_{i}\right)<K(\mu(\varepsilon)+\tilde{g}(\mu(\varepsilon))), \quad i=0, \ldots, n$,

where $\tilde{K}=\frac{K_{2}}{K_{1}} \mathrm{e}^{K_{1}}$, and $n=\left\lfloor\mu^{-1}\right\rfloor$ depends on $\varepsilon$, for the increasing sequence of parameters $t_{i}=i \mu \in[0,1], i=0, \ldots, n$. The points $\tilde{x}_{i}$ represent an orbit of $\tilde{\sigma}_{\varepsilon}$ in $\Lambda$; therefore the points $x_{i}=k_{\varepsilon}\left(\tilde{x}_{i}\right)$ represent an orbit of $\sigma_{\varepsilon}$ in $\Lambda_{\varepsilon}$ satisfying $d\left(x_{i}, \gamma_{\varepsilon}\left(t_{i}\right)\right)<K(\mu(\varepsilon)+\tilde{g}(\mu(\varepsilon)))$, where $\gamma_{\varepsilon}=k_{\varepsilon} \circ \tilde{\gamma}$ and $K$ is a new constant. This orbit $\tilde{x}_{i}$ lies inside the set $\mathcal{U}_{\gamma_{\varepsilon}}=k_{\varepsilon}\left(\mathcal{U}_{\tilde{\gamma}}\right) \subseteq \Lambda_{\varepsilon}$, where a.e. point is recurrent for $\left(f_{\varepsilon}\right)_{\mid \Lambda_{\varepsilon}}$. See Figure 5.3 .

We now apply Theorem 3.6 for the orbit $\left(x_{i}\right)_{i=0, \ldots, n}$ of the scattering map $\sigma_{\varepsilon}$ on $\Lambda_{\varepsilon}$, where $x_{i}=k_{\varepsilon}\left(\tilde{x}_{i}\right)$, and we obtain that, for any $\delta>0$ there exists an orbit $z_{i+1}=f_{\varepsilon}^{k_{i}}\left(z_{i}\right)$ of $f_{\varepsilon}$, which satisfies $d\left(z_{i}, x_{i}\right)<\delta, i=0, \ldots, n$. Therefore we obtain that

$$
d\left(z_{i}, \gamma_{\varepsilon}\left(t_{i}\right)\right)<\delta+K(\mu(\varepsilon)+\tilde{g}(\mu(\varepsilon)))
$$




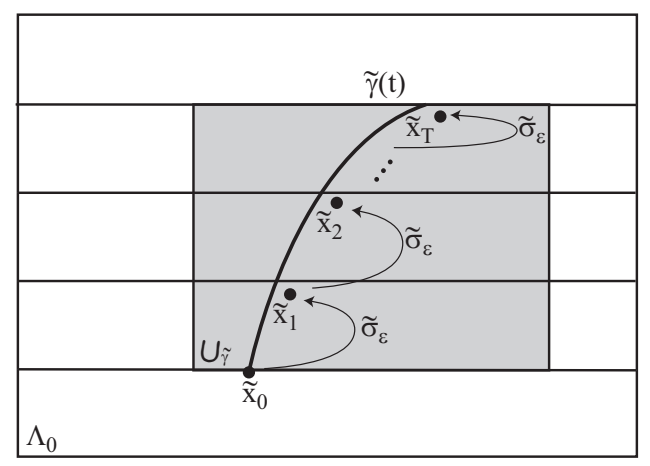

FIGURE 5.3. A scattering path and a nearby orbit of the scattering map.

\subsection{Proof of Corollary 3.12}

By continuity, since $J \nabla S$ is transverse to one level set of the variable $I$ in $\Lambda$, it is transverse to a $O(1)$-family of level sets of the variable $I$. More precisely, there exist two compact disks $D^{d} \subseteq B^{d}, E^{d} \subseteq \mathbb{T}^{d}$, of radii independent of $\varepsilon$, such that $J \nabla S$ is transverse to each level set $\left\{I=I_{a}\right\}$ at $\tilde{\sigma}_{0}\left(I_{a}, \phi_{a}\right)$ for $I_{a} \in D^{d}, \phi_{a} \in E^{d}$.

Let $\Delta=D^{d} \times E^{d}$ and let

$$
\Delta^{\infty}=\bigcup_{n \geq 0} \tilde{f}_{\varepsilon}^{n}(\Delta)
$$

Note that $\Delta \subseteq \Delta^{\infty}$ and that $\Delta^{\infty}$ is positively invariant, i.e., $\tilde{f}_{\varepsilon}\left(\Delta^{\infty}\right) \subseteq \Delta^{\infty}$.

We have the following two possibilities:

I. Either $\mu\left(\Delta^{\infty}\right)=\infty$

II. or $\mu\left(\Delta^{\infty}\right)<\infty$.

Case I implies right away that for every $N>0$, there exists an orbit $\left(\tilde{f}_{\varepsilon}^{n}(\tilde{x})\right)_{n \geq 0}$ of $\tilde{f}_{\varepsilon}$ in $\Lambda$ for which $\left\|I\left(\tilde{f}^{k_{N}}(\tilde{x})\right)-I(\tilde{x})\right\|>N$ for some $k_{N} \geq 0$. It follows immediately that there exist orbits of $f_{\varepsilon}$ as in the statement of the corollary. Notice that in this case we obtain diffusing orbits only by applying the inner dynamics; we do not have to use the scattering map.

Now we consider Case II. Since $\mu\left(\Delta^{\infty}\right)<\infty$ we can apply the Poincaré recurrence theorem, so for every open set $\mathcal{U} \subseteq \Delta$, almost every point of $\mathcal{U}$ is recurrent.

By the assumption on the scattering map, we have that for each $\left(I_{0}, \phi_{0}\right) \in D^{d} \times$ $E^{d}$, the curve $\tilde{\gamma}(t), t \in[0,1]$, obtained by integrating the vector field $J \nabla S$ with initial condition at $\left(I_{0}, \phi_{0}\right)$ is transverse to every level set $\left\{I=I_{a}\right\}$ at a point $\tilde{\gamma}(t)=(I(t), \phi(t))$, where $(I(t), \phi(t)) \in D^{d} \times E^{d}=\Delta$ for all $t \in[0,1]$ and all $0<\varepsilon<\varepsilon_{1}$. Thus, there exists $\rho_{0}>0$, independent of $\varepsilon$, such that

$$
\|I(\tilde{\gamma}(1))-I(\tilde{\gamma}(0))\|>\rho_{0} .
$$

Choose an $\varepsilon_{1}$ as in Theorem 3.11 and fix an $\varepsilon \in\left(0, \varepsilon_{1}\right)$. Choose $0<\delta<\rho_{0} / 4$ and restrict $\varepsilon_{1}$ if necessary in such a way that $K(\mu(\varepsilon)+|g(\mu(\varepsilon))| / \mu(\varepsilon)) \leq \delta$ and let 
$\rho=\rho_{0}-4 \delta>0$. Theorem 3.11 implies that there is an orbit $\left(z_{i}\right)_{i=0, \ldots, n}$ of $f_{\mathcal{E}}$ such that $d\left(z_{0}, \gamma_{\varepsilon}(0)\right)<2 \delta$ and $d\left(z_{n}, \gamma_{\varepsilon}(1)\right)<2 \delta$. Thus, we have $\left\|I\left(z_{n}\right)-I\left(z_{0}\right)\right\|>$ $\rho_{0}-4 \delta=\rho$.

\section{Appendix A Normally Hyperbolic Invariant Manifolds and the Scattering Map}

In this section we recall the background on normally hyperbolic invariant manifolds and the definition of the scattering map and its geometric properties.

The main references for normally hyperbolic manifolds are [48, 49,66,80]. Even if the definitions of [48,49,66] are not completely equivalent, the results that we use are very basic and appear in both treatments as well as in several subsequent treatments [3,4]. The properties of the scattering map appear in [36]. Let $f: M \rightarrow$ $M$ be a $C^{r}$ map on a $C^{r}$-differentiable manifold $M$. Assume that there exists a manifold $\Lambda \subseteq M$ that is a normally hyperbolic invariant manifold for $f$. We will assume that the derivatives of $f$ are uniformly continuous and uniformly bounded in a neighborhood of $\Lambda$. This is, of course, automatic if $\Lambda$ is a compact manifold, and many of the results are stated only for compact manifolds, but as remarked in [3, 4, 66], only the uniform continuity and uniform boundedness of derivatives is needed.

We recall that, following [48, 49, 66, 80], we say that a $\Lambda \subset M$ is a hyperbolic manifold if there exists a splitting of the tangent bundle of $T M$ into $D f$-invariant subbundles

$$
T M=E^{u} \oplus E^{s} \oplus T \Lambda,
$$

and there exist a constant $C>0$ and rates

$$
0<\lambda_{+}<\eta_{-} \leq 1 \leq \eta_{+} \leq \mu_{-}
$$

such that for all $x \in \Lambda$ we have

$$
\begin{array}{rlr}
v \in E_{x}^{s} \Leftrightarrow & \left\|D f_{x}^{k}(v)\right\| \leq C \lambda_{+}^{k}\|v\| & \text { for all } k \geq 0, \\
v \in E_{x}^{u} \Leftrightarrow & \left\|D f_{x}^{k}(v)\right\| \leq C \mu_{-}^{-k}\|v\| & \text { for all } k \leq 0, \\
v \in T_{x} \Lambda \Leftrightarrow & \left\|D f_{x}^{k}(v)\right\| \leq C \eta_{+}^{k}\|v\|, & \\
& \left\|D f_{x}^{-k}(v)\right\| \leq C \eta_{-}^{-k}\|v\| & \text { for all } k \geq 0 .
\end{array}
$$

If $D f(x)$ and $D f^{-1}(x)$ are uniformly bounded, we have that there are opposite inequalities; namely, there exist $\lambda_{-} \leq \lambda_{+}$and $\mu_{+} \geq \mu_{-}$such that

$$
\begin{aligned}
& v \in E_{x}^{s} \Longrightarrow\left\|D f_{x}^{k}(v)\right\| \geq C \lambda_{-}^{k}\|v\| \quad \text { for all } k \geq 0, \\
& v \in E_{x}^{u} \Longrightarrow\left\|D f_{x}^{k}(v)\right\| \geq C \mu_{+}^{-k}\|v\| \quad \text { for all } k \leq 0 .
\end{aligned}
$$

Note that, of course, if the inequalities (A.2) and (A.3) hold for some rates, they also hold for other rates $\tilde{\lambda}_{ \pm}, \tilde{\mu}_{ \pm}$, and $\tilde{\eta}_{ \pm}$satisfying (A.1) such that

$$
\left[\lambda_{-}, \lambda_{+}\right] \subset\left[\tilde{\lambda}_{-}, \tilde{\lambda}_{+}\right], \quad\left[\mu_{-}, \mu_{+}\right] \subset\left[\tilde{\mu}_{-}, \tilde{\mu}_{+}\right], \quad\left[\eta_{-}, \eta_{+}\right] \subset\left[\tilde{\eta}_{-}, \tilde{\eta}_{+}\right]
$$


Clearly, the bounds for $\tilde{\lambda}_{ \pm}, \tilde{\mu}_{ \pm} \cdot \tilde{\eta}_{ \pm}$are less sharp than those for the original values. If we change the metric in the manifold $M$ by an equivalent metric, the rates $\lambda_{ \pm}, \eta_{ \pm}, \mu_{ \pm}$are not altered, but the constant $C$ can be modified. A standard construction [66] shows that, for any rates that satisfy (A.4) with strict inclusions, we can find a metric (called an adapted metric) equivalent to and as smooth as the original one in such a way that $C=1$ both in (A.2) and in (A.3). See [17] for a discussion of adapted metrics for (A.3). Hence, for theoretical purposes (including in this paper) one can assume that $C=1$ in both A.2 and (A.3).

In the case when $f$ is symplectic, it is natural to consider hyperbolic manifolds with the property that

$$
\eta_{-}=1 / \eta_{+}, \quad \lambda_{+}=1 / \mu_{-}, \quad \text { and also } \quad \lambda_{-}=1 / \mu_{+} .
$$

As shown in [36], normally hyperbolic invariant manifolds for symplectic maps with the restricted exponents as in A.5 enjoy many geometric properties (e.g., the map restricted to the manifold is symplectic). Note, however, that even for symplectic maps, there are normally hyperbolic invariant manifolds that satisfy the general definition but not A.5. A notable example is the stable manifold of a NHIM, which is normally hyperbolic according to the general definition (this plays an important role in [48]) but does not satisfy (A.5], and, indeed, the map restricted to it is not symplectic.

Assume that there exists an integer $\ell>0$ such that

$$
\ell \leq \min \left(r, \log \lambda_{-}^{-1} / \log \eta_{+}^{-1}, \log \eta_{-} / \log \mu_{+}\right) .
$$

Then $\Lambda$ is $C^{\ell}$-differentiable, and its stable and unstable manifolds $W^{s}(\Lambda)$ and $W^{u}(\Lambda)$ are $C^{\ell}$-differentiable manifolds. See [84].

The manifolds $W^{s}(\Lambda)$ and $W^{u}(\Lambda)$ are foliated by stable and unstable manifolds of points $W^{s}(z)$ and $W^{u}\left(z^{\prime}\right)$, respectively, with $z, z^{\prime} \in \Lambda$, which are $C^{r}$ differentiable manifolds. The foliations are $C^{\ell-1}$-differentiable. For each $x \in$ $W^{s}(\Lambda)$ there exists a unique $x^{+} \in \Lambda$ such that $x \in W^{s}\left(x^{+}\right)$, and for each $x \in W^{u}(\Lambda)$ there exists a unique $x^{-} \in \Lambda$ such that $x \in W^{u}\left(x^{-}\right)$. We define the wave maps

$$
\begin{aligned}
& \Omega^{+}: W^{s}(\Lambda) \rightarrow \Lambda \text { by } \Omega^{+}(x)=x^{+}, \\
& \Omega^{-}: W^{u}(\Lambda) \rightarrow \Lambda \text { by } \Omega^{-}(x)=x^{-} .
\end{aligned}
$$

The maps $\Omega^{+}$and $\Omega^{-}$are $C^{\ell-1}$-smooth.

We assume that there exists a transverse homoclinic manifold $\Gamma \subseteq M$, which is $C^{\ell-1}$-differentiable. This means that $\Gamma \subseteq W^{u}(\Lambda) \cap W^{s}(\Lambda)$ and, for each $x \in \Gamma$, we have

$$
\begin{aligned}
T_{x} M & =T_{x} W^{u}(\Lambda)+T_{x} W^{s}(\Lambda), \\
T_{x} \Gamma & =T_{x} W^{u}(\Lambda) \cap T_{x} W^{s}(\Lambda) .
\end{aligned}
$$


We assume the additional conditions that for each $x \in \Gamma$ we have

$$
\begin{aligned}
& T_{x} W^{s}(\Lambda)=T_{x} W^{s}\left(x^{+}\right) \oplus T_{x} \Gamma, \\
& T_{x} W^{u}(\Lambda)=T_{x} W^{u}\left(x^{-}\right) \oplus T_{x} \Gamma,
\end{aligned}
$$

where $x^{-}, x^{+}$are the uniquely defined points in $\Lambda$ corresponding to $x$; in this case we say that $\Gamma$ is transverse to the foliations. Following [36], we call $\Gamma$ a homoclinic channel if it is transverse to the foliation, and $\Omega^{-}, \Omega^{+}$restricted to $\Gamma$ are diffeomorphisms. Hence, we can define a scattering map

$$
\sigma: \Omega^{-}(\Gamma) \rightarrow \Omega^{+}(\Gamma), \quad \sigma=\Omega^{+} \circ\left(\Omega^{-}\right)^{-1},
$$

which is a diffeomorphism from $\Omega^{-}(\Gamma)$ to $\Omega^{+}(\Gamma)$.

If $\sigma\left(x^{-}\right)=x^{+}$, then there exists a unique $x \in \Gamma$ such that $W^{u}\left(x^{-}\right) \cap W^{s}\left(x^{+}\right) \cap$ $\Gamma=\{x\}$. Note that the backwards orbit $f^{-n}(x)$ of $x$ in $M$ is asymptotic to the backwards orbit $f^{-n}\left(x^{-}\right)$in $\Lambda$, and the forward orbit $f^{m}(x)$ of $x$ in $M$ is asymptotic to the forward orbit $f^{m}\left(x^{+}\right)$in $\Lambda$.

\section{Appendix B Linearized Coordinates}

We will construct all windows used in Section 5.1.1 in linearized coordinates, which will be recalled below, following [83].

Let $\Lambda$ be a normally hyperbolic invariant manifold for $f$ in $M$. There exists an open neighborhood $V_{\Lambda}$ of $\Lambda$ in $M$, an open neighborhood $U_{\Lambda}$ of the zero section of $\left(E^{u} \oplus E^{s}\right)_{\mid \Lambda}$, and a homeomorphism $h$ from $U_{\Lambda}$ to $V_{\Lambda}$ such that for every $\left(x^{c}, v^{u}, v^{s}\right) \in\left(E^{u} \oplus E^{s}\right)_{\mid \Lambda}$

$$
\begin{aligned}
\left(h^{-1} \circ f \circ h\right)\left(x^{c}, v^{u}, v^{s}\right) & =N f\left(x^{c}, v^{u}, v^{s}\right) \\
& =\left(f_{\mid \Lambda}\left(x^{c}\right), D f\left(x^{c}\right)_{\mid E^{u} \oplus E^{s}}\left(v^{u}, v^{s}\right)\right) .
\end{aligned}
$$

Via this coordinate system, each point $p \in V_{\Lambda}$ can be written uniquely through $\left(x^{c}, v^{u}, v^{s}\right)$ for some $x^{c} \in \Lambda, v^{u} \in E^{u}, v^{s} \in E^{s}$, as $p=h\left(x^{c}, v^{u}, v^{s}\right)$.

In the linearized coordinates, the map $f$ is conjugate with the normal mapping $N f_{\mid E^{u} \oplus E^{s}}$ of $f$ in a neighborhood of $\Lambda$. Hence, iterating a rectangle in these coordinates by the map $f$ for an arbitrary number of times is equivalent to iterating the rectangle by the normal mapping $N f$.

\section{Appendix C Correctly Aligned Windows}

We review briefly the topological method of correctly aligned windows. We follow [94] (see also [54,58]).

DEFINITION C.1. An $\left(m_{1}, m_{2}\right)$-window in an $m$-dimensional manifold $M$, where $m_{1}+m_{2}=m$, is a a $C^{0}$-homeomorphism $\chi$ from some open neighborhood $\operatorname{dom}(\chi)$ of $[0,1]^{m_{1}} \times[0,1]^{m_{2}}$ in $\mathbb{R}^{m_{1}} \times \mathbb{R}^{m_{2}}$ to an open subset $\operatorname{im}(\chi)$ of $M$, together with the homeomorphic image $W=\chi\left([0,1]^{m_{1}} \times[0,1]^{m_{2}}\right)$, and with a choice of an "exit set"

$$
W^{\text {exit }}=\chi\left(\partial[0,1]^{m_{1}} \times[0,1]^{m_{2}}\right)
$$


and of an "entry set"

$$
W^{\text {entry }}=\chi\left([0,1]^{m_{1}} \times \partial[0,1]^{m_{2}}\right) .
$$

Remark C.2. Alternatively, we can define a window as a $C^{0}$-family of $m_{1}$-dimensional disks attached to an $m_{2}$-dimensional disk, i.e.,

$$
W=\bigcup_{q \in D^{m_{1}}} D^{m_{2}}(q),
$$

with $D^{m_{1}}$ being some fixed $m_{2}$-dimensional disk, and $D^{m_{2}}(q)$ being $m_{1}$-dimensional disks depending in a $C^{0}$-fashion on $q \in D^{m_{1}}$, in which case

$$
W^{\text {exit }}=\bigcup_{q \in D^{m_{1}}} \partial D^{m_{2}}(q) \text { and } W^{\text {entry }}=\bigcup_{q \in \partial D^{m_{1}}} D^{m_{2}}(q) \text {. }
$$

In the sequel, when we refer to a window we mean the set $W$ together with the underlying local parametrization $\chi$.

DeFinition C.3. Let $W_{1}$ and $W_{2}$ be $\left(m_{1}, m_{2}\right)$-windows, and let $\chi_{1}$ and $\chi_{2}$ be the corresponding local parametrizations. Let $f$ be a continuous map on $M$ with $f\left(\operatorname{im}\left(\chi_{1}\right)\right) \subseteq \operatorname{im}\left(\chi_{2}\right)$, and let $f_{\chi}=\chi_{2}^{-1} \circ f \circ \chi_{1}$. We say that $W_{1}$ is correctly aligned with $W_{2}$ under $f$ if the following conditions are satisfied:

(i) There exists a continuous homotopy $h:[0,1] \times\left([0,1]^{m_{1}} \times[0,1]^{m_{2}}\right) \rightarrow$ $\mathbb{R}^{m_{1}} \times \mathbb{R}^{m_{2}}$, such that the following conditions hold true:

$$
\begin{aligned}
h_{0} & =f_{\chi}, \\
h\left([0,1], \partial[0,1]^{m_{1}} \times[0,1]^{m_{2}}\right) \cap\left([0,1]^{m_{1}} \times[0,1]^{m_{2}}\right) & =\varnothing, \\
h\left([0,1],[0,1]^{m_{1}} \times[0,1]^{m_{2}}\right) \cap\left([0,1]^{m_{1}} \times \partial[0,1]^{m_{2}}\right) & =\varnothing .
\end{aligned}
$$

(ii) There exists $y_{0} \in[0,1]^{m_{2}}$ such that the map $A_{y_{0}}:[0,1]^{m_{1}} \rightarrow \mathbb{R}^{m_{1}}$ defined by $A_{y_{0}}(x)=\pi_{m_{1}}\left(h_{1}\left(x, y_{0}\right)\right)$ satisfies

$$
A_{y_{0}}\left(\partial[0,1]^{m_{1}}\right) \subseteq \mathbb{R}^{m_{1}} \backslash[0,1]^{m_{1}}, \quad \operatorname{deg}\left(A_{y_{0}}, 0\right) \neq 0,
$$

where $\pi_{m_{1}}: \mathbb{R}^{m_{1}} \times \mathbb{R}^{m_{2}} \rightarrow \mathbb{R}^{m_{1}}$ is the projection onto the first component, and $\operatorname{deg}(\cdot, 0)$ is the Brouwer degree of a map at 0 .

The following is a shadowing-lemma type of result for correctly aligned windows.

THEOREM C.4. Let $f: M \rightarrow M$ be a homeomorphism, $W_{i}$ be a collection of $\left(m_{1}, m_{2}\right)$-windows in $M$, and $\left\{t_{i}\right\}$ be a collection of positive integers, where $i \in \mathbb{Z}$. If $W_{i}$ is correctly aligned with $W_{i+1}$ under $f^{t_{i}}$ for each $i$, then there exists a point $p \in W_{0}$ such that

$$
\left(f^{t_{i}} \circ \cdots \circ f^{t_{0}}\right)(p) \in W_{i+1} \quad \text { for all } i .
$$

Moreover, iffor some $k>0$ we have $t_{i+k}=t_{i}$ and $W_{i+k}=W_{i}$ for all $i$, then the point $p$ can be chosen periodic with period $t_{0}+\cdots+t_{k-1}$. 
The correct alignment satisfies a natural product property. Given two windows and a map, if each window can be written as a product of window components, and if the components of the first window are correctly aligned with the corresponding components of the second window under the appropriate components of the map, then the first window is correctly aligned with the second window under the given map. The details can be found in [54].

We describe the product property in a special case, which corresponds to the situation considered in the paper.

Let $f: M \rightarrow M$ be a homeomorphism of the $m$-dimensional manifold $M$. Denote by $B_{\rho}^{k}(x)$ the $k$-dimensional closed ball of radius $\rho$ centered at the point $x$ in $\mathbb{R}^{k}$. Assume that $c, u, s \in \mathbb{N}$ are such that $c+u+s=m$, and write each $x \in \mathbb{R}^{m}$ as $x=\left(x^{c}, x^{u}, x^{s}\right)$, with $x^{c} \in \mathbb{R}^{c}, x^{u} \in \mathbb{R}^{u}$, and $x^{s} \in \mathbb{R}^{s}$. Let $p_{1}, p_{2}$ be two points in $M$, and let $\chi_{1}, \chi_{2}$ be two systems of local coordinates about $p_{1}, p_{2}$, respectively. Relative to these coordinate systems, we write $p_{1}=\left(p_{1}^{c}, p_{1}^{u}, p_{1}^{s}\right)$ and $p_{2}=\left(p_{2}^{c}, p_{2}^{u}, p_{2}^{s}\right)$.

LEMMA C.5. Given two sets, $W_{1}$ in the local chart around $p_{1}$, and $W_{2}$ in the local chart around $p_{2}$ such that, in the corresponding local coordinates, we have

$$
\begin{aligned}
& W_{1}=B_{\rho_{1}^{c}}^{c}\left(p_{1}^{c}\right) \times B_{\rho_{1}^{u}}^{u}\left(p_{1}^{u}\right) \times B_{\rho_{1}^{s}}^{s}\left(p_{1}^{s}\right), \\
& W_{2}=B_{\rho_{2}^{c}}^{c}\left(p_{2}^{c}\right) \times B_{\rho_{2}^{u}}^{u}\left(p_{2}^{u}\right) \times B_{\rho_{1}^{s}}^{s}\left(p_{2}^{s}\right),
\end{aligned}
$$

for some $\rho_{1}^{c}, \rho_{1}^{u}, \rho_{1}^{s}, \rho_{2}^{c}, \rho_{2}^{s}, \rho_{2}^{u}>0$. Let

$$
\begin{aligned}
W_{1}^{\text {exit }}= & \partial B_{\rho_{1}^{c}}^{c}\left(p_{1}^{c}\right) \times B_{\rho_{1}^{u}}^{u}\left(p_{1}^{u}\right) \times B_{\rho_{1}^{s}}^{s}\left(p_{1}^{s}\right) \\
& \cup B_{\rho_{1}^{c}}^{c}\left(p_{1}^{c}\right) \times \partial B_{\rho_{1}^{u}}^{u}\left(p_{1}^{u}\right) \times B_{\rho_{1}^{s}}^{s}\left(p_{1}^{s}\right), \\
W_{1}^{\text {entry }}= & B_{\rho_{1}^{c}}^{c}\left(p_{1}^{c}\right) \times B_{\rho_{1}^{u}}^{u}\left(p_{1}^{u}\right) \times \partial B_{\rho_{1}^{s}}^{s}\left(p_{1}^{s}\right), \\
W_{2}^{\text {exit }}= & \partial B_{\rho_{2}^{c}}^{c}\left(p_{2}^{c}\right) \times B_{\rho_{2}^{u}}^{u}\left(p_{2}^{u}\right) \times B_{\rho_{2}^{s}}^{s}\left(p_{2}^{s}\right) \\
& \cup B_{\rho_{2}^{c}}^{c}\left(p_{2}^{c}\right) \times \partial B_{\rho_{2}^{u}}^{u}\left(p_{2}^{u}\right) \times B_{\rho_{2}^{s}}^{s}\left(p_{2}^{s}\right), \\
W_{2}^{\text {entry }}= & B_{\rho_{2}^{c}}^{c}\left(p_{2}^{c}\right) \times B_{\rho_{2}^{u}}^{u}\left(p_{2}^{u}\right) \times \partial B_{\rho_{2}^{s}}^{s}\left(p_{2}^{s}\right) .
\end{aligned}
$$

Assume that the map $f$, written in local coordinates, satisfies the following conditions relative to $W_{1}$ and $W_{2}$ :

$$
\begin{aligned}
& \pi_{c} \circ f\left(B_{\rho_{1}^{c}}^{c}\left(p_{1}^{c}\right) \times\left\{p_{1}^{u}\right\} \times\left\{p_{1}^{s}\right\}\right) \supseteq B_{\rho_{2}^{c}}^{c}\left(p_{2}^{c}\right), \\
& \pi_{u} \circ f\left(\left\{p_{1}^{c}\right\} \times B_{\rho_{1}^{u}}^{u}\left(p_{1}^{u}\right) \times\left\{p_{1}^{s}\right\}\right) \supseteq B_{\rho_{2}^{u}}^{u}\left(p_{2}^{u}\right), \\
& \pi_{s} \circ f\left(\left\{p_{1}^{c}\right\} \times\left\{p_{1}^{u}\right\} \times B_{\rho_{1}^{s}}^{s}\left(p_{1}^{s}\right)\right) \subseteq B_{\rho_{2}^{s}}^{s}\left(p_{2}^{s}\right),
\end{aligned}
$$

where $\pi_{c}, \pi_{u}, \pi_{s}$ denote the standard projections onto $\mathbb{R}^{c}, \mathbb{R}^{u}, \mathbb{R}^{s}$, respectively.

Then $W_{1}$ and $W_{2}$ are $(c+u, s)$-windows, and $W_{1}$ is correctly aligned with $W_{2}$ under $f$.

This lemma is an immediate consequence of proposition 3 in [54]. 


\section{Appendix D An Example of D. Turaev}

We are very grateful to Dmitry Turaev who provided to us an example that shows that a "uniform-time" version of the shadowing lemma analogue of Lemma 3.1 is not true in general.

This example shows that the requirement that

$$
m_{i} \geq m_{i}^{*}\left(n_{0}, \ldots, n_{i-1}, m_{0}, \ldots, m_{i-1}\right)
$$

in Lemma 3.1 cannot be replaced by $m_{i} \geq m^{*}$, where $m^{*}$ is a constant.

Example D.1. Let $M=\mathbb{R}^{3}, f: M \rightarrow M$ be a $C^{1}$-map, and $\Lambda$ be a straight line in $M$ that is a normally hyperbolic invariant manifold for $f$ as follows. There exists a system of coordinates $(x, u, v)$ in a neighborhood $V$ of $\Lambda$ in $M$, with $x \in \mathbb{R}$ representing the coordinate on $\Lambda$, and $u \in \mathbb{R}$ and $v \in \mathbb{R}$ the contracting and expanding directions, respectively, and a corresponding open set $U \subseteq \mathbb{R}^{3}$ of the form

$$
U=\{(x, u, v): x \in \mathbb{R},|u|<3 / 2,|v|<3 / 2\}
$$

such that for $p=(x, u, v) \in U$, the map $f$ is of the form $f(x, u, v)=\left(x^{\prime}, u^{\prime}, v^{\prime}\right)$ where

$$
x^{\prime}=x+(u v)^{2}, \quad u^{\prime}=u / 2, \quad v^{\prime}=2 v .
$$

Thus $\Lambda$ corresponds to $u=v=0$, and for each point $p=(x, 0,0) \in \Lambda$, $W^{s}(p)=\{(x, u, 0)\}$, and $W^{u}(p)=\{(x, 0, v)\}$. Moreover, $f_{\mid \Lambda}=\mathrm{Id}$, and $f\left(W^{s, u}(p)\right)=W^{s, u}(p)$ for every $p \in \Lambda$, that is, $f$ leaves invariant the stable and unstable fibers.

Assume that $W^{u}(\Lambda)$ and $W^{s}(\Lambda)$ intersect transversally along a homoclinic manifold

$$
\Gamma^{-}=\{(x, u, v): u=0, v=1\},
$$

which is a line, and that for some power $q>0$ the map $f^{q}$ is of the form $f^{q}(x, u, v)=\left(x^{\prime \prime}, u^{\prime \prime}, v^{\prime \prime}\right)$,

$$
x^{\prime \prime}=x+1, \quad u^{\prime \prime}=1+u, \quad v^{\prime \prime}=v-1 .
$$

Thus $f^{q}\left(\Gamma^{-}\right)=\Gamma^{+}=\{(x, u, v): u=1, v=0\}$, and the corresponding scattering map $\sigma: \Lambda \rightarrow \Lambda$ is of the form

$$
\sigma(x, 0,0)=(x+1,0,0) .
$$

Assume that for every $\delta>0$ there exists $n^{*}$ such that for every pseudo-orbit $y_{i+1}=f^{n_{i}} \circ \sigma\left(y_{i}\right)$ with $n_{i} \geq n^{*}$ there exists a true orbit $z_{i+1}=f^{n_{i}}\left(z_{i}\right)$ such that $d\left(z_{i}, y_{i}\right)<\delta$ for all $i$. Take $\delta>0$ small and a corresponding $n^{*}$ sufficiently large. Choose and fix a pseudo-orbit $y_{i+1}=f^{n_{i}} \circ \sigma\left(y_{i}\right)$ with $n_{i}=n=n^{*}$ for all $i \geq 0$. Let $y_{i}=\left(x_{i}, 0,0\right)$. Since $f_{\mid \Lambda}=\operatorname{Id}$ and $\sigma$ shifts the $x$-coordinate by 1 unit, we have

$$
x_{i+1}=x_{i}+1
$$


Assume that there is an orbit $z_{i+1}=f^{n}\left(z_{i}\right)$ whose points are $\delta$-close to the corresponding points $y_{i}$. Let $z_{i}=\left(x_{i}^{\prime}, u_{i}, v_{i}\right)$. Shadowing would imply that for all $i \geq 0$ we have

$$
\left|x_{i}^{\prime}-x_{i}\right|<\delta, \quad\left|u_{i}\right|<\delta, \quad\left|v_{i}\right|<\delta .
$$

First we show that one of the iterations $f^{k}\left(z_{i}\right)$ with $0<k<n$ must lie outside the neighborhood $U$ of $\Lambda$ (i.e., the orbit between $z_{i}$ and $z_{i+1}$ makes an excursion along the homoclinic). Indeed, note that as long as the orbit of $z_{i}$ lies in the neighborhood $U$, the product $u v$ stays constant by (D.1), so if the orbit between $z_{i}$ and $z_{i+1}$ stays in $U$ for all time, then

$$
x_{i+1}^{\prime}=x_{i}^{\prime}+n\left(u_{i} v_{i}\right)^{2} .
$$

Also, by (D.1), we must have $\left|v_{i}\right|<\frac{3}{2^{n+1}}$, so $\left|x_{i+1}^{\prime}-x_{i}^{\prime}\right|<\frac{9 n}{4^{n+1}} u_{i}^{2}$, which contradicts (D.4) and (D.5) if $\delta$ is small. Thus, the orbit between $z_{i}$ and $z_{i+1}$ must leave at some point $f^{\overline{k+1}}\left(z_{i}\right)$ the neighborhood $U$.

Thus, for each $i \geq 0$ there exists a positive integer $k_{i}<n-q$ such that the first $k_{i}$ iterations of $z_{i}$ stay in $U$, the next $q$ iterations stay outside $U$ following the homoclinic and returning to $U$, and the last $n-q-k_{i}$ iterations stay in $U$ again. For the first $k_{i}$ iterations the product $u v$ stays equal to $u_{i} v_{i}$, and for the last $n-q-k_{i}$ iterations the product stays constant and equals $u_{i+1} v_{i+1}$ (see $(\overline{\mathrm{D} .1})$ ). Thus, by (D.1) and (D.2),

$$
x_{i+1}^{\prime}=x_{i}^{\prime}+1+k_{i}\left(u_{i} v_{i}\right)^{2}+\left(n-q-k_{i}\right)\left(u_{i+1} v_{i+1}\right)^{2}
$$

Using (D.1), since $z_{i}$ leaves $U$ after $k_{i}<n-q$ forward iterations, we have

$$
\left|v_{i}\right|>3 / 2^{n-q+2} \text { for all } i,
$$

and since $z_{i}$ leaves $U$ after $n-q-k_{i} \leq n-q$ negative iterations,

$$
\left|u_{i}\right|>3 / 2^{n-q+2} \text { for all } i \text {. }
$$

Therefore,

$$
\left(u_{i} v_{i}\right)^{2}>1 / 2^{4 n} \text { for all } i
$$

hence (D.6) implies

$$
x_{i+1}^{\prime}>x_{i}^{\prime}+1+(n-q) / 2^{4 n} \geq x_{i}^{\prime}+1+1 / 2^{4 n} ;
$$

thus

$$
x_{i+1}^{\prime}>x_{0}^{\prime}+i+i\left(1 / 2^{4 n}\right) .
$$

By (D.4)

$$
x_{i}=x_{0}+i,
$$

the distance between $x_{i}^{\prime}$ and $x_{i}$ grows without bound as $i$ grows, for any choice of $x_{0}$ and $x_{0}^{\prime}$, so the shadowing property will be broken after finitely many iterations for any choice of the constant $n$. 
Remark D.2. The idea of this counterexample is that the dynamics off $\Lambda$ differs from the dynamics restricted to $\Lambda$ by some fixed amount of shift that depends on the $u$-and $v$-coordinates of a point. Thus the shift between the points of the pseudo-orbit (which lie on $\Lambda$ ) and the points of a shadowing orbit that takes the same number of iterates between successive points keeps increasing by the fixed amount of shift at every step, and the pseudo-orbit and the shadowing orbit end up being far apart. If we allow that the number of iterates between successive points of the pseudo-orbit to vary, as is the case in Lemma 3.1, we can arrange that the shadowing orbit gets closer and closer to $\Lambda$, which makes the amount of shift between its points in $\Lambda$ and the points of the pseudo-orbit smaller and smaller at every step. More precisely, in the above example where we consider a shadowing orbit $z_{i+1}=f^{n_{i}}\left(z_{i}\right)$ with $n_{i}$ sufficiently large and depending on $i$, the above estimates yield an error term between $x_{i+1}^{\prime}$ and $x_{i+1}$ of the order

$$
\sum_{k=0}^{i} n_{i} / 2^{4 n_{i}},
$$

which can be made arbitrarily small by choosing, for instance, $n_{0}$ sufficiently large and $n_{i}$ increasing at a linear rate.

Remark D.3. The proof of Lemma 3.1 uses the existence of a linearized system of coordinates $h$ in a neighborhood of $\Lambda$ (cf. [83]). In Example D.1] such a system of coordinates is given by

$$
h(x, u, v)= \begin{cases}\left(x+\frac{\ln |u|-\ln |v|}{2 \ln 2}(u v)^{2}, u, v\right), & \text { for } u, v \neq 0, \\ (x, u, 0), & \text { for } v \neq 0, \\ (x, 0, v), & \text { for } u \neq 0, \\ (x, 0,0), & \text { for } u=v=0 .\end{cases}
$$

Indeed, note that

$$
\begin{aligned}
h \circ f(x, u, v) & =h\left(x+(u v)^{2}, u / 2,2 v\right) \\
& =\left(x+(u v)^{2}+\left(\frac{\ln |u|-\ln |v|}{2 \ln 2}-1\right)(u v)^{2}, u / 2,2 v\right) \\
& =\left(x+\left(\frac{\ln |u|-\ln |v|}{2 \ln 2}\right)(u v)^{2}, u / 2,2 v\right) \\
& =N f \circ h(x, u, v) .
\end{aligned}
$$

Acknowledgments. We thank M. Capiński, A. Delshams, P. Martín, and P. Roldán for discussions and comments. I. Baldomá helped to prepare the figures. We especially thank D. Turaev for comments on a previous version and for communicating to us his very clarifying example in Appendix D.

The research of M.G. was partially supported by National Science Foundation Grant DMS-0635607, and by Alfred P. Sloan Foundation Grant G-2016-7320. The 
research of R.L. was partly supported by National Science Foundation Grant DMS1800241. The research of T.S. was partially supported by the MINECO-FEDER Grants MTM2015-65715-P and MdM-2014-0445, Catalan Grant 2017SGR1049, the Russian Scientific Foundation Grant 14-41-00044, and the ICREA Academia 2019 award.

\section{Bibliography}

[1] Arnold, V. Instability of dynamical systems with several degrees of freedom. Sov. Math. Doklady 5 (1964), 581-585. 1.1

[2] Baldomá, I.; Fontich, E.; Guardia, M.; Seara, T. M. Exponentially small splitting of separatrices beyond Melnikov analysis: rigorous results. J. Differential Equations 253 (2012), no. 12, 33043439. doi:10.1016/j.jde.2012.09.0033.16

[3] Bates, P. W.; Lu, K.; Zeng, C. Invariant foliations near normally hyperbolic invariant manifolds for semiflows. Trans. Amer. Math. Soc. 352 (2000), no. 10, 4641-4676. doi:10.1090/S00029947-00-02503-4 A

[4] Bates, P. W.; Lu, K.; Zeng, C. Approximately invariant manifolds and global dynamics of spike states. Invent. Math. 174 (2008), no. 2, 355-433. doi:10.1007/s00222-008-0141-y 5.4. A

[5] Bernard, P. The dynamics of pseudographs in convex Hamiltonian systems. J. Amer. Math. Soc. 21 (2008), no. 3, 615-669. doi:10.1090/S0894-0347-08-00591-2 1.2

[6] Bernard, P.; Kaloshin, V.; Zhang, K. Arnold diffusion in arbitrary degrees of freedom and normally hyperbolic invariant cylinders. Acta Math. 217 (2016), no. 1, 1-79. doi:10.1007/s11511016-0141-5 5, $1.2,3.10$

[7] Berti, M.; Biasco, L.; Bolle, P. Drift in phase space: a new variational mechanism with optimal diffusion time. J. Math. Pures Appl. (9) 82 (2003), no. 6, 613-664. doi:10.1016/S00217824(03)00032-1[1.2

[8] Berti, M.; Bolle, P. Fast Arnold diffusion in systems with three time scales. Discrete Contin. Dyn. Syst. 8 (2002), no. 3, 795-811. doi:10.3934/dcds.2002.8.795 1.2

[9] Berti, M.; Bolle, P. A functional analysis approach to Arnold diffusion. Ann. Inst. H. Poincaré Anal. Non Linéaire 19 (2002), no. 4, 395-450. doi:10.1016/S0294-1449(01)00084-1]1.2

[10] Bessi, U. An approach to Arnol'd's diffusion through the calculus of variations. Nonlinear Anal. 26 (1996), no. 6, 1115-1135. doi:10.1016/0362-546X(94)00270-R 1.2

[11] Bessi, U. Arnold's example with three rotators. Nonlinearity 10 (1997), no. 3, 763-781. doi:10.1088/0951-7715/10/3/010 1.2

[12] Bessi, U.; Chierchia, L.; Valdinoci, E. Upper bounds on Arnold diffusion times via Mather theory. J. Math. Pures Appl. (9) 80 (2001), no. 1, 105-129. doi:10.1016/S0021-7824(00)0118801.2

[13] Bolotin, S. Symbolic dynamics of almost collision orbits and skew products of symplectic maps. Nonlinearity 19 (2006), no. 9, 2041-2063. doi:10.1088/0951-7715/19/9/003 3.2

[14] Bolotin, S.; MacKay, R. S. Nonplanar second species periodic and chaotic trajectories for the circular restricted three-body problem. Celestial Mech. Dynam. Astronom. 94 (2006), no. 4, 433-449. doi:10.1007/s10569-006-9006-0 3.2

[15] Bolotin, S.; Treschev, D. Unbounded growth of energy in nonautonomous Hamiltonian systems. Nonlinearity 12 (1999), no. 2, 365-388. doi:10.1088/0951-7715/12/2/013 1.2

[16] Bounemoura, A. An example of instability in high-dimensional Hamiltonian systems. Int. Math. Res. Not. IMRN (2012), no. 3, 685-716. doi:10.1093/imrn/rnr043 1.2

[17] Cabré, X.; Fontich, E.; Llave, R. d. 1. The parameterization method for invariant manifolds. II. Regularity with respect to parameters. Indiana Univ. Math. J. 52 (2003), no. 2, 329-360. doi:10.1512/iumj.2003.52.2407A 
[18] Canalias, E.; Delshams, A.; Masdemont, J. J.; Roldán, P. The scattering map in the planar restricted three body problem. Celestial Mech. Dynam. Astronom. 95 (2006), no. 1-4, 155-171. doi:10.1007/s10569-006-9010-4/2.1]

[19] Capinski, M.; Gidea, M.; de la Llave, R. Arnold diffusion in the planar elliptic restricted threebody problem: mechanism and numerical verification. Nonlinearity 30 (2017), no. 1, 329-360. doi:10.1088/1361-6544/30/1/32944,2.1,3.8, 4.2

[20] Cheng, C.-Q. Arnold diffusion in nearly integrable Hamiltonian systems. Preprint, 2012. arXiv:1207.4016 [math.DS 1.2

[21] Cheng, C.-Q.; Xue, J. Arnold diffusion in nearly integrable hamiltonian systems of arbitrary degrees of freedom. Preprint, 2015. arXiv:1503.04153 [math.DS] 1.2

[22] Cheng, C.-Q.; Yan, J. Existence of diffusion orbits in a priori unstable Hamiltonian systems. J. Differential Geom. 67 (2004), no. 3, 457-517. 1.23 .9

[23] Cheng, C.-Q.; Yan, J. Arnold diffusion in Hamiltonian systems: a priori unstable case. J. Differential Geom. 82 (2009), no. 2, 229-277. 1.23 .9

[24] Chierchia, L.; Gallavotti, G. Drift and diffusion in phase space. Ann. Inst. H. Poincaré Phys. Théor. 60 (1994), no. 1, 144 pp.1.1

[25] Chirikov, B. V. A universal instability of many-dimensional oscillator systems. Phys. Rep. 52 (1979), no. 5, 264-379. doi:10.1016/0370-1573(79)90023-1[1.2

[26] Cresson, J.; Guillet, C. Hyperbolicity versus partial-hyperbolicity and the transversalitytorsion phenomenon. J. Differential Equations 244 (2008), no. 9, 2123-2132. doi:10.1016/j.jde.2008.02.009 5.1.1.C

[27] Cresson, J.; Wiggins, S. A $\lambda$-lemma for normally hyperbolic invariant manifolds. Regul. Chaotic Dyn. 20 (2015), no. 1, 94-108. doi:10.1134/S15603547150100745.1.1.C

[28] de la Llave, R. Orbits of unbounded energy in perturbations of geodesic flows by periodic potentials. a simple construction. Preprint, 2004.1.2

[29] de la Llave, R.; Olvera, A.; Petrov, N. P. Combination laws for scaling exponents and relation to the geometry of renormalization operators. J. Statist. Phys. 143 (2011), no. 5, 889-920. doi:10.1007/s10955-011-0211-15.4

[30] del-Castillo-Negrete, D.; Greene, J. M.; Morrison, P. J. Renormalization and transition to chaos in area preserving nontwist maps. Phys. D 100 (1997), no. 3-4, 311-329. doi:10.1016/S01672789(96)00200-X 3.2, 4.4

[31] Delshams, A.; de la Llave, R. KAM theory and a partial justification of Greene's criterion for nontwist maps. SIAM J. Math. Anal. 31 (2000), no. 6, 1235-1269. doi:10.1137/S003614109834908X 2.1, 3.2, 4.4

[32] Delshams, A.; de la Llave, R.; Seara, T. M. A geometric approach to the existence of orbits with unbounded energy in generic periodic perturbations by a potential of generic geodesic flows of

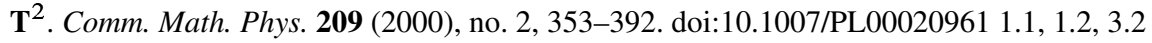

[33] Delshams, A.; de la Llave, R.; Seara, T. M. A geometric mechanism for diffusion in Hamiltonian systems overcoming the large gap problem: announcement of results. Electron. Res. Announc. Amer. Math. Soc. 9 (2003), 125-134. doi:10.1090/S1079-6762-03-00121-5[1.2

[34] Delshams, A.; de la Llave, R.; Seara, T. M. A geometric mechanism for diffusion in Hamiltonian systems overcoming the large gap problem: heuristics and rigorous verification on a model. Mem. Amer. Math. Soc. 179 (2006), no. 844, viii+141 pp. doi:10.1090/memo/0844 $1.2,2.1$ 2.2. $2.2,4,4.2,4$

[35] Delshams, A.; de la Llave, R.; Seara, T. M. Orbits of unbounded energy in quasi-periodic perturbations of geodesic flows. Adv. Math. 202 (2006), no. 1, 64-188. doi:10.1016/j.aim.2005.03.005 2.1

[36] Delshams, A.; de la Llave, R.; Seara, T. M. Geometric properties of the scattering map of a normally hyperbolic invariant manifold. Adv. Math. 217 (2008), no. 3, 1096-1153. doi:10.1016/j.aim.2007.08.014 1.1, 2.1, 2.2, 2.2, 3.2, 4, A A A 
[37] Delshams, A.; de la Llave, R.; Seara, T. M. Instability of high dimensional Hamiltonian systems: multiple resonances do not impede diffusion. Adv. Math. 294 (2016), 689-755. doi:10.1016/j.aim.2015.11.010 $1.2444 .2,4$

[38] Delshams, A.; Gidea, M.; de la Llave, R.; Seara, T. M. Geometric approaches to the problem of instability in Hamiltonian systems. An informal presentation. Hamiltonian dynamical systems and applications, 285-336. NATO Science for Peace and Security Series B: Physics and Biophysics. Springer, Dordrecht, 2008. doi:10.1007/978-1-4020-6964-2_13]1.2, 2.2,4

[39] Delshams, A.; Gidea, M.; Roldan, P. Transition map and shadowing lemma for normally hyperbolic invariant manifolds. Discrete and Continuous Dynamical Systems. Series A. 3 (2013), no. 33, 1089-1112. doi:10.3934/dcds.2013.33.1089 2.1] 3.55 .3

[40] Delshams, A.; Gidea, M.; Roldan, P. Arnold's mechanism of diffusion in the spatial circular restricted three-body problem: a semi-analytical argument. Phys. D 334 (2016), 29-48. doi:10.1016/j.physd.2016.06.005 4 2.1, 3.8 . 5.3

[41] Delshams, A.; Gutiérrez, P. Homoclinic orbits to invariant tori in Hamiltonian systems. Multiple-time-scale dynamical systems (Minneapolis, MN, 1997), 1-27. The IMA Volumes in Mathematics and Its Applications, 122. Springer, New York, 2001. doi:10.1007/978-1-46130117-2_13.13

[42] Delshams, A.; Huguet, G. Geography of resonances and Arnold diffusion in a priori unstable Hamiltonian systems. Nonlinearity 22 (2009), no. 8, 1997-2077. doi:10.1088/09517715/22/8/013 1.2, 4, 4.2

[43] Delshams, A.; Kaloshin, V.; de la Rosa, A.; Seara, T. Global instability in the restricted planar elliptic three body problem. Comm. Math. Phys., 2018. doi:10.1007/s00220-018-3248-z 3.8,4

[44] Delshams, A.; Schaefer, R. G. Arnold diffusion for a complete family of perturbations . Regul. Chaotic Dyn. 22 (2017), no. 1, 78-108. doi:10.1134/S15603547170100514,4

[45] Delshams, A.; Schaefer, R. G. Arnold diffusion for a complete family of perturbations with two independent harmonics. Discrete Contin. Dyn. Syst. 38(12) (2018), 6047-6072. 4

[46] Eliasson, L. H. Biasymptotic solutions of perturbed integrable Hamiltonian systems. Bol. Soc. Brasil. Mat. (N.S.) 25 (1994), no. 1, 57-76. doi:10.1007/BF01232935]3.13

[47] Féjoz, J.; Guardia, M.; Kaloshin, V.; Roldán, P. Kirkwood gaps and diffusion along mean motion resonances in the restricted planar three-body problem. J. Eur. Math. Soc. (JEMS) 18 (2016), no. 10, 2315-2403. doi:10.4171/JEMS/642 3.8

[48] Fenichel, N. Persistence and smoothness of invariant manifolds for flows. Indiana Univ. Math. J. 21 (1971/1972), 193-226. doi:10.1512/iumj.1971.21.21017 A A

[49] Fenichel, N. Asymptotic stability with rate conditions. Indiana Univ. Math. J. 23 (1973/74), 1109-1137. doi:10.1512/iumj.1974.23.23090 2.1,3.13, A

[50] Fontich, E.; Martín, P. Differentiable invariant manifolds for partially hyperbolic tori and a lambda lemma. Nonlinearity 13 (2000), no. 5, 1561-1593. doi:10.1088/0951-7715/13/5/309 5.1.1.C

[51] Gelfreich, V.; Turaev, D. Unbounded energy growth in Hamiltonian systems with a slowly varying parameter. Comm. Math. Phys. 283 (2008), no. 3, 769-794. doi:10.1007/s00220-0080518-1 1.2

[52] Gelfreich, V.; Turaev, D. Arnold diffusion in a priori chaotic symplectic maps. Comm. Math. Phys. 353 (2017), no. 2, 507-547. doi:10.1007/s00220-017-2867-0 1.2 3.5 5.3

[53] Gidea, M.; de la Llave, R. Arnold diffusion with optimal time in the large gap problem. Preprint, 2006. $1.2,4$

[54] Gidea, M.; de la Llave, R. Topological methods in the instability problem of Hamiltonian systems. Discrete Contin. Dyn. Syst. 14 (2006), no. 2, 295-328.1.2,3.2, C C C

[55] Gidea, M.; de la Llave, R. Global Melnikov theory in Hamiltonian systems with general timedependent perturbations. J. Nonlinear Sci. 28 (2018), no. 5, 1657-1707.2.2 2.2. 3.9 3.13

[56] Gidea, M.; de la Llave, R. Perturbations of geodesic flows by recurrent dynamics. J. Eur. Math. Soc. (JEMS) 19 (2017), no. 3, 905-956. doi:10.4171/JEMS/683 $1.23 .2,3.9$ 
[57] Gidea, M.; Marco, J.-P. Diffusion along chains of normally hyperbolic cylinders. Preprint, 2017. arXiv: 1708.08314[math.DS] 1.2

[58] Gidea, M.; Robinson, C. Topologically crossing heteroclinic connections to invariant tori. J. Differential Equations 193 (2003), no. 1, 49-74. doi:10.1016/S0022-0396(03)00065-2 5.1.1.C.IV] C

[59] Gidea, M.; Robinson, C. Shadowing orbits for transition chains of invariant tori alternating with Birkhoff zones of instability. Nonlinearity 20 (2007), no. 5, 1115-1143. doi:10.1088/0951$7715 / 20 / 5 / 0043.2$

[60] Gidea, M.; Robinson, C. Diffusion along transition chains of invariant tori and Aubry-Mather sets. Ergodic Theory Dynam. Systems 33 (2013), 1401-1449. doi:10.1017/S0143385712000363 $3.54 .2,5.3$

[61] Granados, A. Invariant manifolds and the parameterization method in coupled energy harvesting piezoelectric oscillators. Phys. D 351/352 (2017), 14-29. doi:10.1016/j.physd.2017.04.003]1.2

[62] Granados, A.; Hogan, S. J.; Seara, T. M. The scattering map in two coupled piecewisesmooth systems, with numerical application to rocking blocks. Phys. D 269 (2014), 1-20. doi:10.1016/j.physd.2013.11.0083.10

[63] Haller, G. Universal homoclinic bifurcations and chaos near double resonances. J. Statist. Phys. 86 (1997), no. 5-6, 1011-1051. doi:10.1007/BF02183612 1.2

[64] Haller, G. Chaos near resonance. Applied Mathematical Sciences, 138. Springer, New York, 1999. doi:10.1007/978-1-4612-1508-0[1.2

[65] Hirsch, M.; Palis, J.; Pugh, C.; Shub, M. Neighborhoods of hyperbolic sets. Invent. Math. 9 (1969/1970), 121-134. doi:10.1007/BF01404552 5.1.1.C

[66] Hirsch, M.; Pugh, C.; Shub, M. Invariant manifolds. Lecture Notes in Mathematics, 583. Springer, Berlin, 1977.3.13, A, A

[67] Kaloshin, V. Geometric proofs of Mather's connecting and accelerating theorems. Topics in dynamics and ergodic theory, 81-106. London Mathematical Society Lecture Note Series, 310. Cambridge University Press, Cambridge, 2003. doi:10.1017/CBO9780511546716.007[1.2

[68] Kaloshin, V.; Zhang, K. A strong form of Arnold diffusion for two and a half degrees of freedom. Preprint, 2012. Available at: https://www.math.umd.edu/ vkaloshi/ papers/announce-three-and-half .pdf $1.2,3.10$

[69] Kaloshin, V.; Zhang, K. Normally hyperbolic invariant manifolds near strong double resonance. Preprint, 2012. arXiv:1202.1032 [math.DS] 5, 1.2$] 3.10$

[70] Kaloshin, V.; Zhang, K. A strong form of Arnold diffusion for three and a half degrees of freedom. Available at: https://www.math.umd.edu/ vkaloshi/papers/ announce-three-and-half.pdf 1.2

[71] Lieberman, M. A.; Tennyson, J. L. Chaotic motion along resonance layers in near-integrable Hamiltonian systems with three or more degrees of freedom. Long-time prediction in dynamics (Lakeway, Tex., 1981), 179-211. Nonequilib. Problems Phys. Sci. Biol., 2. Wiley, New York, 1983. 1.2

[72] Lochak, P.; Marco, J.-P.; Sauzin, D. On the splitting of invariant manifolds in multidimensional near-integrable Hamiltonian systems. Mem. Amer. Math. Soc. 163 (2003), no. 775, viii+145 pp. doi:10.1090/memo/0775/3.13

[73] Luque, A.; Peralta-Salas, D. Arnold diffusion of charged particles in ABC magnetic fields. $J$. Nonlinear Sci. 27 (2017), no. 3, 721-774. doi:10.1007/s00332-016-9349-y 1.2

[74] Marco, J.-P. Modèles pour les applications fibrées et les polysystèmes. C. R. Math. Acad. Sci. Paris 346 (2008), no. 3-4, 203-208. doi:10.1016/j.crma.2007.11.017 3.2

[75] Marco, J.-P. Generic hyperbolic properties of classical systems on the torus. Preprint, 2013.5. 1.2

[76] Mather, J. N. Order structure on action minimizing orbits. Symplectic topology and measure preserving dynamical systems, 41-125, Contemporary Mathematics, 512. American Mathematical Society, Providence, R.I., 2010. doi:10.1090/conm/512/10060 1.2 
[77] Mather, J. N. Arnold diffusion by variational methods. Essays in mathematics and its applications, 271-285. Springer, Heidelberg, 2012. doi:10.1007/978-3-642-28821-0_11 1.2$] 3.10$

[78] Mèzer, Dzh. N. Arnol'd diffusion. I. Announcement of results. Sovrem. Mat. Fundam. Napravl. 2 (2003), 116-130; translation in J. Math. Sci. (N.Y.) 124 (2004), no. 5, 5275-5289. doi:10.1023/B:JOTH.0000047353.78307.09 1.2

[79] Nassiri, M.; Pujals, E. R. Robust transitivity in Hamiltonian dynamics. Ann. Sci. Éc. Norm. Supér. (4) 45 (2012), no. 2, 191-239. doi:10.24033/asens.2164[1.2

[80] Pesin, Y. B. Lectures on partial hyperbolicity and stable ergodicity. Zurich Lectures in Advanced Mathematics. European Mathematical Society (EMS), Zürich, 2004. doi:10.4171/00:A

[81] Piftankin, G. N. Diffusion speed in the Mather problem. Dokl. Akad. Nauk 408 (2006), no. 6, 736-737.1.2

[82] Piftankin, G. N.; Treshchëv, D. V. Separatrix maps in Hamiltonian systems. Uspekhi Mat. Nauk 62 (2007), no. 2(374), 3-108; translation in Russian Math. Surveys 62 (2007), no. 2, 219-322. doi:10.1070/RM2007v062n02ABEH004396 1.2

[83] Pugh, C.; Shub, M. Linearization of normally hyperbolic diffeomorphisms and flows. Invent. Math. 10 (1970), 187-198. doi:10.1007/BF01403247/B]D.3

[84] Robinson, C. Differentiable conjugacy near compact invariant manifolds. Bol. Soc. Brasil. Mat. 2 (1971), no. 1, 33-44. doi:10.1007/BF02584805 A

[85] Sabbagh, L. An inclination lemma for normally hyperbolic manifolds with an application to diffusion. Ergodic Theory Dynam. Systems 35 (2015), no. 7, 2269-2291. doi:10.1017/etds.2014.30 5.1.1.C

[86] Shatah, J.; Zeng, C. Orbits homoclinic to centre manifolds of conservative PDEs. Nonlinearity 16 (2003), no. 2, 591-614. doi:10.1088/0951-7715/16/2/314 5.4

[87] Takens, F. Homoclinic points in conservative systems. Invent. Math. 18 (1972), 267-292. doi:10.1007/BF013898163.13

[88] Tennyson, J. Resonance transport in near-integrable systems with many degrees of freedom. Phys. D 5 (1982), no. 1, 123-135. doi:10.1016/0167-2789(82)90054-9 1.2

[89] Treschev, D. Multidimensional symplectic separatrix maps. J. Nonlinear Sci. 12 (2002), no. 1, 27-58. doi:10.1007/s00332-001-0460-2 1.2

[90] Treschev, D. Trajectories in a neighbourhood of asymptotic surfaces of a priori unstable Hamiltonian systems. Nonlinearity 15 (2002), no. 6, 2033-2052. doi:10.1088/0951-7715/15/6/313 1.2

[91] Treschev, D. Evolution of slow variables in a priori unstable Hamiltonian systems. Nonlinearity 17 (2004), no. 5, 1803-1841. doi:10.1088/0951-7715/17/5/014 1.2

[92] Treschev, D. Arnold diffusion far from strong resonances in multidimensional a priori unstable Hamiltonian systems. Nonlinearity 25 (2012), no. 9, 2717-2757. doi:10.1088/0951$7715 / 25 / 9 / 27171.2$

[93] Xia, Z. Homoclinic points in symplectic and volume-preserving diffeomorphisms. Comm. Math. Phys. 177 (1996), no. 2, 435-449.3.13

[94] Zgliczyński, P.; Gidea, M. Covering relations for multidimensional dynamical systems. J. Differential Equations 202 (2004), no. 1, 32-58. doi:10.1016/j.jde.2004.03.013|C

[95] Zhang, K. Speed of Arnold diffusion for analytic Hamiltonian systems. Invent. Math. 186 (2011), no. 2, 255-290. doi:10.1007/s00222-011-0319-6 1.2 
MARIAN GIDEA

Yeshiva University

Department of Mathematical Sciences

New York, NY 10016

USA

E-mail: Marian.Gidea@yu.edu

TERE M-SEARA

Universitat Politècnica de Catalunya

Departament de Matemàtiques

Diagonal 647, 08028 Barcelona

SPAIN

E-mail: Tere.M-Seara@upc.edu

Received February 2018.
RAFAEL DE LA LLAVE Georgia Institute of Technology

School of Mathematics

Atlanta, GA 30332

USA

E-mail: rafael.delallaved

math.gatech.edu 